\title{
The Wendelstein Calar Alto Pixellensing Project (WeCAPP): the M 31 nova catalogue
}

\author{
C.-H. Lee ${ }^{1}$, A. Riffeser ${ }^{1}$, S. Seitz ${ }^{1,2}$, R. Bender ${ }^{1,2}$, J. Fliri ${ }^{3}$, U. Hopp ${ }^{1,2}$, C. Ries ${ }^{1}$, O. Bärnbantner ${ }^{1}$, and C. Gössl ${ }^{1}$ \\ 1 University Observatory Munich, Scheinerstrasse 1, 81679 München, Germany \\ e-mail: chlee@usm.lmu.de \\ 2 Max Planck Institute for Extraterrestrial Physics, Giessenbachstrasse, 85748 Garching, Germany \\ 3 GEPI, CNRS UMR 8111, Observatoire de Paris, 92195 Meudon, France
}

Received 12 April 2011 / Accepted 22 September 2011

\begin{abstract}
We present light curves from the novae detected in the long-term, M 31-monitoring WeCAPP project. The goal of WeCAPP is to constrain the compact dark matter fraction of the M 31 halo with microlensing observations. As a by product we detected 91 novae benefiting from the high cadence and highly sensitive difference imaging technique required for pixel-lensing. We thus can now present the largest sample of optical/CCD nova lightcurves towards M 31 to date. We also obtained thorough coverage of the light curve before and after the eruption thanks to the long-term monitoring. We apply a nova taxonomy to our nova candidates and found 29 S-class novae, $10 \mathrm{C}$-class novae, $2 \mathrm{O}$-class novae, and $1 \mathrm{~J}$-class nova. We investigated a universal decline law on the S-class novae. In addition, we correlated our catalogue with the literature and found 4 potential recurrent novae. Part of our catalogue has been used to search for optical counterparts of the super soft X-ray sources detected in M 31. Optical surveys like WeCAPP, when coordinated with multi-wavelength observation, will continue to shed light on the underlying physical mechanism of novae in the future.
\end{abstract}

Key words. galaxies: individual: M 31 - novae, cataclysmic variables

\section{Introduction}

Classical novae span a subclass of cataclysmic variables, consisting of a white dwarf that interacts with a late-type companion star. The companion loses its mass through Roche lobe overflow, forming an accretion disk around the white dwarf. The mass transfer from the companion induces thermo-nuclear runaway (TNR) onto the surface of the white dwarf, which leads to the nova eruption.

Novae are important in several aspects. First of all, they have the potential of serving as standard candles of extragalactic distance indication. This is due to the relation between the maximum luminosity of the light curve and the rate of decline. Hubble (1929) first noticed that brighter novae are prone to steeper decline. The empirical "Maximum Magnitude versus Rate of Decline" (MMRD) relation was further investigated by Zwicky (1936) and studied quantitatively by Mclaughlin (1945) and Arp (1956). The theoretical foundation for MMRD relation was laid down by Shara (1981) and further revised by Livio (1992).

Novae could also shed light on the underlying stellar population of the environment. For example, della Valle \& Livio (1995) proposed that fast novae $\left(t_{2}<12\right.$ days $)$ are related to stars belonging to Population I with relatively massive white dwarfs, while slow novae are associated to Population II stars and have less massive white dwarfs.

In addition, novae play a role in the galactic abundances. They have been considered as major sources of galactic ${ }^{13} \mathrm{C},{ }^{15} \mathrm{~N}$, and ${ }^{17} \mathrm{O}$, and minor contributors to ${ }^{7} \mathrm{Li},{ }^{9} \mathrm{~F}$, and ${ }^{26} \mathrm{Al}$. However, novae hardly contribute to the overall galactic metallicity compared to supernovae or AGB stars, because only $10^{-4}$ to $10^{-5} \mathrm{M}_{\odot}$ are ejected per nova outburst (José \& Hernanz 2007).
Recurrent novae are also regarded as possible supernovae progenitor candidates (see e.g., Schaefer 2010, and reference therein.). The fundamental question is whether recurrent novae accumulate enough mass onto the central white dwarf envelope and turn into supernovae progenitors even after several novae explosions.

Last but not the least, novae are main contributors to the class of super soft X-ray sources (SSS). Pietsch et al. (2005) searched for X-ray counterparts of the optical novae in M 31, and found that novae are major sources of soft X-ray emission. The SSS phase can provide us with information on the white dwarf, the ejected and the burned mass in the outburst (e.g., Pietsch 2010).

Due to the interstellar extinction in the Galactic disk, we can only observe a small fraction of the Galactic novae that erupt each year (Shafter 1997). Thus, we need to take rather large (and likely uncertain) corrections for incompleteness into account when determining the spatial distribution or estimation of the Galactic nova rate. In this case, M 31 is an ideal target for a novae survey because novae are still bright enough to be observed $\left(m_{R}<20 \mathrm{mag}\right)$, and it is possible to cover the entire $\mathrm{M} 31$ galaxy within several pointings.

Novae monitoring campaigns towards M 31 can be dated back to the pioneering work done by Hubble in the 1920s (Hubble 1929). A list of all the campaigns with published novae in M 31 that we are aware of is shown in Table 1, with most of the data compiled by Shafter \& Irby (2001) and Darnley et al. (2004).

Despite the extensive search towards M 31, most previous studies only have sparse observations and thus make the analysis of nova light curve rather difficult. Our WeCAPP project is dedicated to monitoring M 31 with up to daily sampling for 
Table 1. Principal M 31 classical nova surveys.

\begin{tabular}{lcccccc}
\hline \hline Author(s)/Project & Epoch & Filter(s) & Detector & Novae & Annual rate & Reference(s) \\
\hline Hubble & $1909-1927$ & $B$ & Plates & 85 & $\sim 30$ & Hubble (1929) \\
Arp & $1953-1954$ & $B$ & Plates & 30 & $26 \pm 4$ & Arp (1956) \\
Rosino et al. & $1955-1986$ & $B$ & Plates & 142 & - & Rosino (1964, 1973); Rosino et al. (1989) \\
Ciardullo et al. & $1982-1986$ & $B, \mathrm{H} \alpha$ & CCD & 40 & - & Ciardullo et al. (1987, 1990) \\
Sharov \& Alksins & $1969-1989$ & $B$ & Plates & 21 & - & Sharov \& Alksnis (1991) \\
Tomaney \& Shafter & $1987-1989$ & $\mathrm{H} \alpha$ & CCD & 9 & - & Tomaney \& Shafter (1992) \\
Shafter \& Irby & $1990-1997$ & $\mathrm{H} \alpha$ & $\mathrm{CCD}$ & 72 & $37_{-8}^{+12}$ & Shafter \& Irby (2001) \\
Rector et al. & $1995-1999$ & $\mathrm{H} \alpha$ & $\mathrm{CCD}$ & 44 & - & Rector et al. (1999b) \\
AGAPE & $1994-1996$ & $R, I$ & CCD & 12 & - & Ansari et al. (2004) \\
POINT-AGAPE & $1999-2002$ & $r^{\prime}, i^{\prime}, g^{\prime}$ & CCD & 20 & $65_{-15}^{+16}$ & Darnley et al. (2006) \\
NMS & $2001-2002$ & $R, I$ & CCD & 2 & - & Joshi et al. (2004) \\
WeCAPP & $1997-2008$ & $R, I$ & CCD & 91 & - & This work \\
\hline
\end{tabular}

a duration of 11 years. The use of two filters ( $R$ and $I$-band) for the novae observations partly compensates for not having used an $\mathrm{H} \alpha$ filter as in previous studies. Instead of observing the $\mathrm{H} \alpha$ strength we can at least measure the colour evolution of the novae.

This paper is organized as follows. In Sect. 2 we describe the observations and data reduction. In Sect. 3 we present our novae detection algorithm. In Sect. 4 we categorize our nova candidates according to the classification scheme of Strope et al. (2010). We apply the power-law decline proposed by Hachisu \& Kato (2006) to fit the smooth class light curves in Sect. 4.1. Novae showing cusp, oscillation, or jitter features in their light curves are presented in Sects. 4.2-4.4. We then correlate our nova candidates with the literature to search for recurrent novae in Sect. 5. We show the rate of decline of our nova candidates and the distribution of their speed class in Sect. 6 and end the paper with the conclusions in Sect. 7. All the light curves in our catalogue are presented in the Appendix.

\section{Observations and data reduction}

The WeCAPP project (Riffeser et al. 2001) was a dedicated survey to search for microlensing events towards our neighbouring galaxy M 31. We continuously monitored the bulge of M 31 (when it was visible, when the weather was cooperative, and when there was an observer) between September 1997 and March 2008 using the $0.8 \mathrm{~m}$ telescope of the Wendelstein Observatory located in the Bavarian Alps. The data was taken optimally on a daily basis in both $R$ and $I$ filters with a field of view of $8.3 \times 8$ '3. From June 1999 to February 2002, we further extended our observations with the $1.23 \mathrm{~m}(17 ! 2 \times 17 ! 2$ FOV $)$ telescope of the Calar Alto Observatory in Spain. After 2002 we have been using the Wendelstein telescope solely to mosaic the full Calar Alto field of view with four pointings. The position of these four pointing are indicated in Fig. 1.

The data volume and quality of the four pointings (F1-F4) drastically differs during the 11 seasons. A list of the number of nights observed in each season is shown in Table 2. A detailed overview of the observaions can be found in Fliri et al. (2006) and Riffeser et al. (in prep.).

To quantify a realistic time sampling of the survey we define "good quality data points" as data points with PSF fluxes with an error below $0.4 \times 10^{-5} \mathrm{Jy}$. In Fig. 2 we show for every night the fractional area of pixels with errors below this limit. The designation $0 \%$ indicates we have no observations during the night.

Figure 3 shows the spatial variation of the fraction of all data with flux errors below the flux error limit averaged over 11 seasons. It demonstrates that we expect most of our novae in field F1

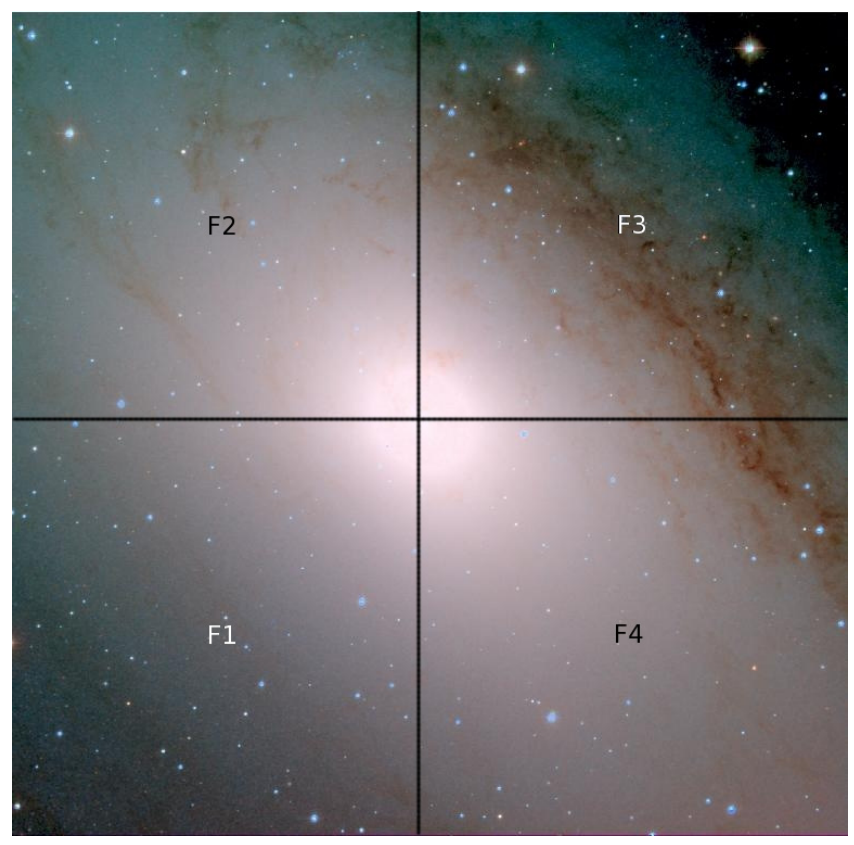

Fig. 1. M 31 composite image ( $V$-, $R$-, and $I$-band) taken at the Calar Alto Observatory. The black lines mark the four pointings (F1 to F4) from the Wendelstein Observatory to mosaic the full FOV of the Calar Alto Observatory.

and fewer in the fields F2-F4. The field F1 was observed much more frequently than the other one because it is the subfield with highest lensing probability.

The data was then reduced by our customized pipeline MUPIPE (see Gössl \& Riffeser 2002), which performs CCD reduction, position alignment, photometric alignment, frame stacking, and difference imaging following the algorithm of Alard \& Lupton (1998).

After the difference imaging, we performed PSF photometry on each pixel as follows. First, we extracted the PSF from several isolated, bright and unsaturated reference stars. Then we fitted this PSF to all variable sources. Finally, we integrated the count rates over the area of the PSF to determine the flux of the source.

The results of the project are presented in Riffeser et al. (2003, 2008), and they partially contributed to Calchi Novati et al. (2010). In addition to the original microlensing targets, the intensive observations in two bands also yield more than 20000 variables in the bulge of M 31 (Fliri et al. 2006) and the nova candidates presented in this paper. 
Table 2. Lists of the analysed nights per season from the 11-year WeCAPP campaign.

\begin{tabular}{lcccccccc}
\hline \hline Season & \multicolumn{3}{c}{$R$-band } & \multicolumn{4}{c}{$I$-band } \\
& F1 & F2 & F3 & F4 & F1 & F2 & F3 & F4 \\
\hline $1997-1998$ & 36 & 7 & 1 & 4 & 33 & 7 & 0 & 3 \\
$1998-1999$ & 33 & 1 & 1 & 1 & 28 & 1 & 1 & 1 \\
$1999-2000$ & 154 & 0 & 0 & 0 & 145 & 0 & 0 & 0 \\
$2000-2001$ & 184 & 108 & 124 & 108 & 159 & 89 & 104 & 89 \\
$2001-2002$ & 240 & 136 & 159 & 136 & 212 & 119 & 140 & 119 \\
$2002-2003$ & 34 & 18 & 24 & 18 & 30 & 16 & 24 & 18 \\
$2003-2004$ & 35 & 24 & 29 & 31 & 33 & 21 & 26 & 29 \\
$2004-2005$ & 25 & 23 & 26 & 25 & 19 & 16 & 19 & 19 \\
$2005-2006$ & 30 & 26 & 28 & 29 & 26 & 20 & 22 & 23 \\
$2006-2007$ & 107 & 106 & 103 & 103 & 48 & 45 & 46 & 47 \\
$2007-2008$ & 62 & 56 & 52 & 58 & 36 & 35 & 35 & 38 \\
\hline Total & 940 & 505 & 547 & 513 & 769 & 369 & 417 & 386 \\
\hline
\end{tabular}

Notes. Each season starts from the 1 May until the 30 April in the next year. The WeCAPP campaigns began in 1997 focusing on F1 with the Wendelstein Observatory. From 1999 until 2002 we extended our observations by including the Calar Alto Observatory, which boosted the number of images taken in these seasons. From 2002 on, we use the Wendelstein Observatory solely and mosaic the full Calar Alto FOV with four pointings.

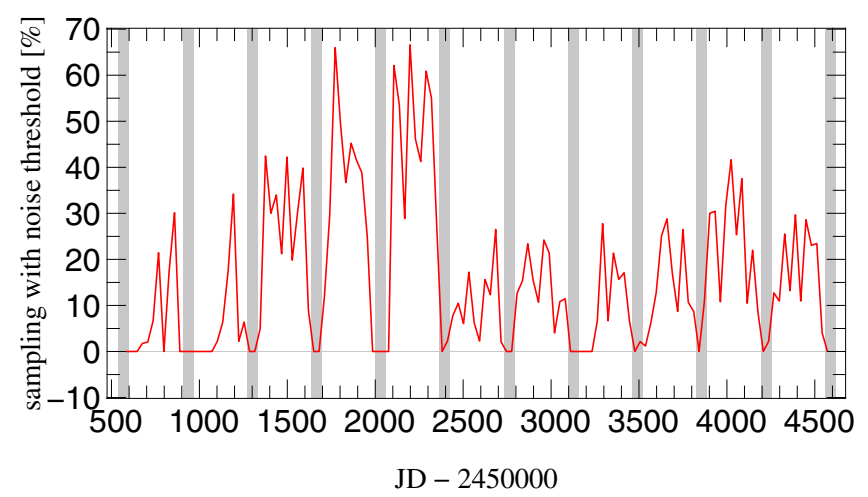

Fig. 2. Fraction of good quality data points in $t$ averaged over the survey area. The definition of good quality is given in the text. The vertical grey zones indicate the time when M 31 is not observable from the location of the telescopes during May and June). As above indicates we had no observations during the night.

\section{Nova detection}

To establish an automatic detection for nova candidates, we applied the following criteria for candidates selection based on the measured $R$-band PSF flux (as mentioned in Sect. 2):

- The significance for variability must be $10 \sigma$ relative to the baseline and the measured flux excess of the variable source must be a local maximum around neighbouring pixels at a given time step. Throughout this paper $\sigma$ refers to the errors of the individual PSF flux excess measurements.

- The variable source must have a measured flux excess larger than $4 \times 10^{-5} \mathrm{Jy}$ in $R$-band (corresponding to $m_{R}=$ $-2.5 \log \left(\frac{4 \times 10^{-5} \mathrm{Jy}}{F_{\mathrm{Vega}, R}}\right) \sim 19.7 \mathrm{mag}$, with $F_{\mathrm{Vega}, R}=3060 \mathrm{Jy}$ being the flux of Vega in the $R$-band), and the first measurement after the measured maximum flux excess must have a flux excess $>2 \times 10^{-5} \mathrm{Jy}$.

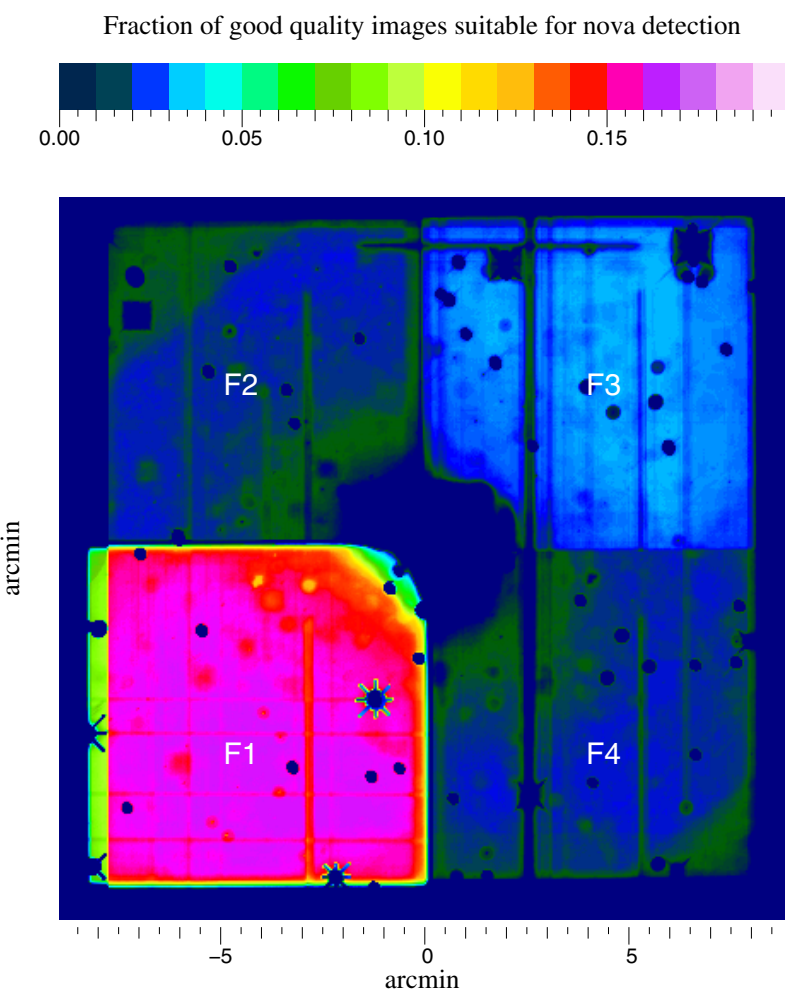

Fig. 3. Fraction of good quality data points in $(x, y)$ averaged over time $t$. The definition of good quality is given in the text. The low fraction in the central part is caused by the high noise of M 31 itself.

- To use the eruptive nature of novae, we define the strength $s$ of the outburst:

$s=\frac{\Delta F_{\max } / \sigma_{\max }^{2}+\Delta F_{\max +1} / \sigma_{\max +1}^{2}}{1 / \sigma_{\max }^{2}+1 / \sigma_{\max +1}^{2}}$

where $\Delta F_{\max }$ is the measured maximum flux excess relative to the reference image, and $\Delta F_{\max +1}$ is the first measurement after the measured maximum flux excess. The $\sigma_{\max }$ and $\sigma_{\max +1}$ are the errors in the measurements of the flux excess. We require $s>4.6 \times 10^{-5} \mathrm{Jy}$ for nova detection.

- To avoid false contamination from periodically varying sources, we define the asymmetry $a$ between positive and negative outliers in the light curve relative to the baseline as:

$a=\frac{\text { Number of data points with } \Delta F>5 \sigma}{\text { Number of data points with } \Delta F<-5 \sigma}-1$.

This quantity $a$ is useful in filtering out normal variable sources, which have $a \sim 0$, while the eruptive nature of novae lead to $a \gg 1$. We empirically require $a$ to be larger than 4.7 to be suitable for nova detection.

- We than apply a special mask to filter false detections around bright stars, especially spikes.

- After the masking, we apply a group algorithm to find multiple pixel detections connecting to the same nova candidate in different time steps.

- In the last step, we inspect the difference images and light curves by eye to make sure that no image artefact escapes our detection and is misinterpreted as a nova.

We combine the criteria 1-4 into one single step. The detections filtered out by each step are shown in Table 3 . 
Table 3. Detection criteria for nova candidates.

\begin{tabular}{lc}
\hline \hline Criterion & Number \\
\hline Full light curves & 4043256 \\
Local flux maximum with & \\
$s>4.6 \times 10^{-5}$ Jy and $a>4.7$ & 1005 \\
Masking of bright stars & 156 \\
Grouping & 105 \\
Inspection by eye & 91 \\
\hline
\end{tabular}

Among the nova candidates, 24 are discovered by WeCAPP project for the first time, while five of them are known but were not officially published and can be found on the $\mathrm{CBAT}^{1}$ or Extragalactic Novae ${ }^{2}$ webpages. The rest of the nova candidates are published and can be found in the literature, see e.g. Pietsch et al. (2007) and Pietsch $(2010)^{3}$. The positions and light curves of these 91 novae are presented in Table 4 and in the Appendix.

\section{Nova taxonomy}

Although all novae differ slightly, it is possible to group novae by their light-curve or spectroscopic properties. One of the commonly used methods of characterizing novae is the "speed class" proposed by Payne-Gaposchkin (1964), who categorized novae according to their light-curve evolution and described the decline timescale by the time needed to drop by two magnitudes below the maximum $\left(t_{2}\right)$. Williams (1992) did a thorough study of the spectroscopic properties of the novae and categorized novae into Fe (galactic thick disk novae) or He (galactic disk novae) group according to the most prominent features in their spectra. Della Valle \& Livio (1998) further established the connection between the speed class and spectroscopic classification. They found that fast novae are mainly related to the He novae, while the slow novae tend to show Fe II features in their spectra. The prospoed explanation behind is that He novae are from the galactic disk and prone to have massive white dwarfs, thus having fast and steep decline. On the other hand, the Fe II novae originate in the less massive population II stars in the galactic thick disk, and hence have a slow decline.

The speed class is not enough to fully account for the differences between novae. Strope et al. (2010) gathered 93 galactic novae from the American Association of Variable Star Observers (AAVSO) and made a thorough study using the complete coverage of their light curves.

They suggested classifying the novae according to their distinct features during their decline, such as the plateau, the cusp by the secondary brightening, and the dip by the dust.

In this section we classify our nova candidates (if possible) following the taxonomy proposed by Strope et al. (2010). Readers are referred to Table 3 and Fig. 2 in Strope et al. (2010) for the definition and exemplary light curves for different nova classes. The classification scheme of Strope et al. (2010) is based on the $V$-band magnitude, while we are using $R$-band and might be affected by the strong $\mathrm{H} \alpha$ emission. We thus check our $I$-band light curve, which does not affected by the strong $\mathrm{H} \alpha$ emission, and identify the apparent features in the nova classification scheme of Strope et al. (2010) in both $R$ and $I$-band.

\footnotetext{
1 M 31 (Apparent) Novae Page, http://www.cfa.harvard.edu/ iau/CBAT_M31.html

2 Www .rochesterastronomy.org/novae.html

3 An up-to-date online-version of the catalogue can be found at http://www . mpe.mpg. de/ m31novae/opt/m31/index . php
}

\subsection{S Class and the universal decline law}

The S-class novae have smooth light curves following the universal power-law decline $\left(F \propto t^{-1.75}\right)$ due to free-free emission expanding shell as proposed by Hachisu \& Kato (2006). In principle, the classification scheme of Strope et al. (2010) is based on the fact that all the light curves originate in the S-class. The $\mathrm{S}$-class is indeed consistent to the vast majority of our nova candidates. To verify the universal decline law, we thus fit our candidate light curves with a 4-parameter formula:

$\Delta F=f_{\mathrm{b}}+f_{0} \times\left(t-t_{0}\right)^{\alpha}$,

where $f_{\mathrm{b}}$ is the baseline level and will be different from zero in cases where the nova candidate flux is present in the reference frame used in difference imaging or there is a variable close to it (see e.g., the light curve of WeCAPP-N10 in the Appendix). The term $f_{0}$ gives the proportional factor between the flux and time, $t_{0}$ is the onset of nova outburst and $\alpha$ is the index of the power-law decline. After the first iteration, we found that some candidates have $t_{0}$ unreasonable long before the nova eruption. For such events, we use a 5-parameter formula

$\Delta F=f_{\mathrm{b}}+f_{0} \times\left(t-t_{0}\right)^{\alpha}, \quad t_{0} \equiv t_{-1}+\delta^{2}$

with $t_{-1}$ fixed at the last data point in the baseline just before the eruption to avoid unreasonable $t_{0}$. The best-fit parameters for Eqs. (3) and (4) are given in Table 5.

For the S-class nova, we first tried to fit the power-law decline for all the nova candidates. A candidate is classified as $\mathrm{S}$-class nova only when the fitting routine finds a solution for either Eqs. (3) or (4). N01, N09, N17, N24, N41, N49, N54, N58, and $\mathrm{N} 77$ are not attributed to $\mathrm{S}$-class because the fitting routine failed to find a solution.

Our best-fit value of $\alpha$ from Table 5 for free $t_{0}$ solely and combined with fixed $t_{0}$ are -1.51 and -1.32 , respectively. The power-law index for a free $t_{0}$ is close to the value given by Hachisu \& Kato (2006), while the value of $\alpha$ from a combination of both free and fixed $t_{0}$ deviates from -1.75 , which indicates that we might have missed the true eruption date for some of the novae. We constrain the value of power-law index $\alpha$ from the $R$-band images, which are contaminated by the $\mathrm{H} \alpha$ emission line and might differ from the universal power-law index from Hachisu \& Kato (2006).

\subsection{Class}

The light curves of C-class novae have a cusp shape that first follows a power-law decline, then rise steeply to a second maximum and finally have a sharp drop. The characteristic C-class light curve has a secondary maximum emerging between one to eight months after the primary peak (Strope et al. 2010). Kato et al. (2009) find that a C-class light curve can be well-fitted by an exponential component superimposed on the smooth decline. They further proposed that the cusps can originate in a secondary ejection and the break-out into the optically thick nova winds. Hachisu \& Kato (2009) also connect the formation of the cusp shape to the input of the magnetic energy from rotating white dwarf. In addition, the sharp drop before the light curve returns to the power-law decline is attributed to the sudden formation of dust as proposed by Lynch et al. (2008). We have found in total ten candidates showing cusp features in our WeCAPP catalogue and show their light curves in Fig. 7. 
C.-H. Lee et al.: The Wendelstein Calar Alto Pixellensing Project (WeCAPP): the M 31 nova catalogue

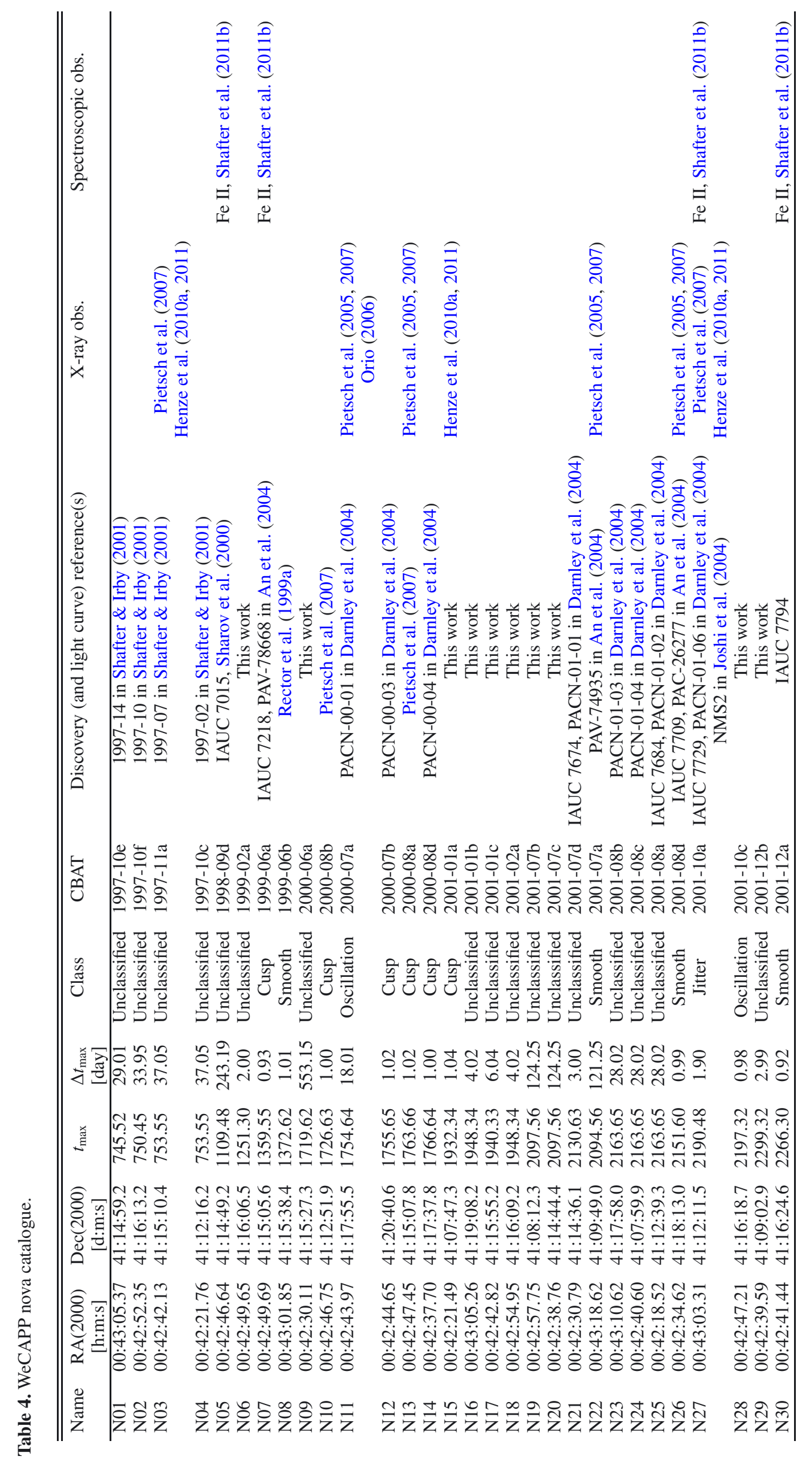

A43, page 5 of 59 


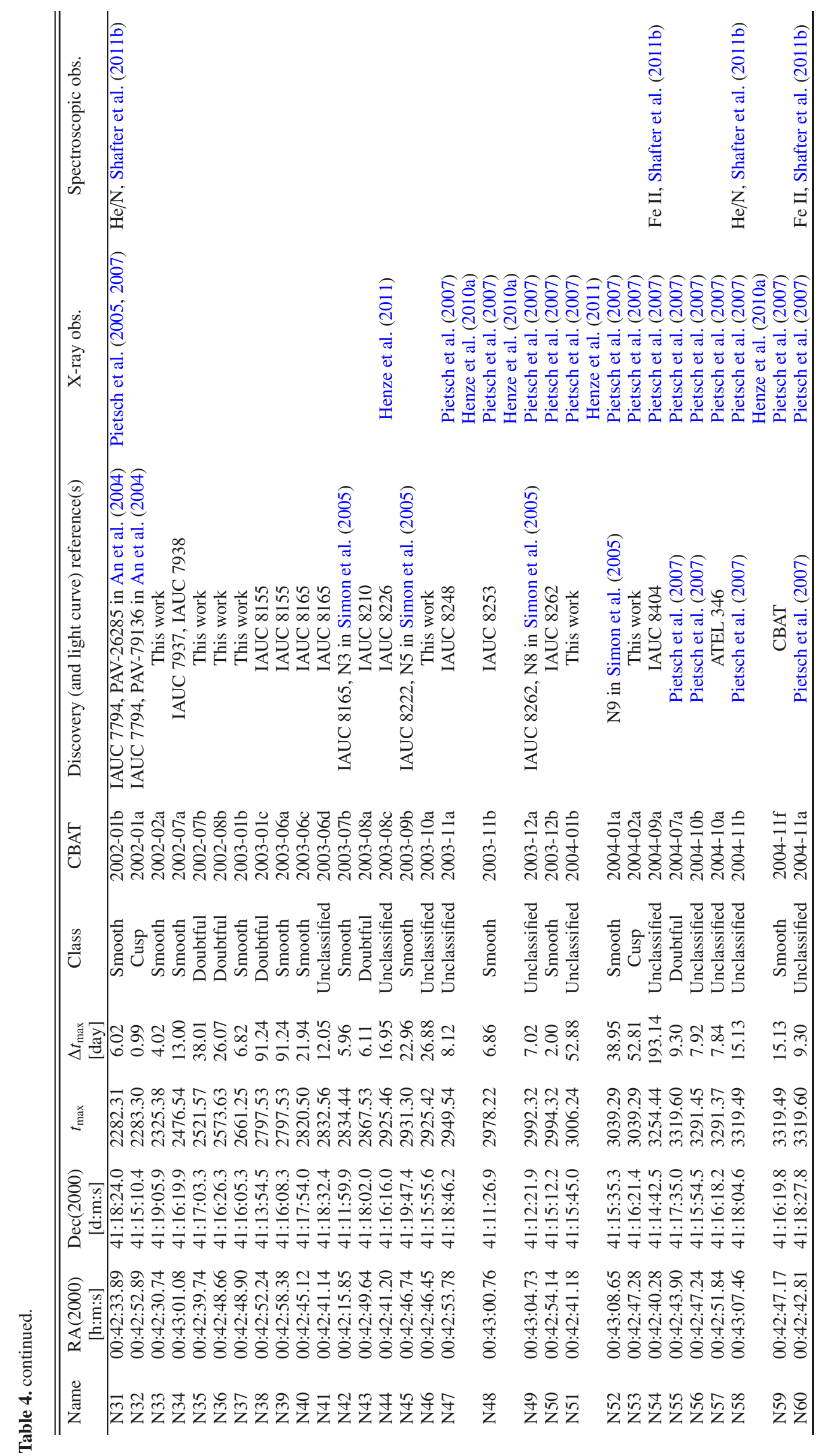


C.-H. Lee et al.: The Wendelstein Calar Alto Pixellensing Project (WeCAPP): the M 31 nova catalogue

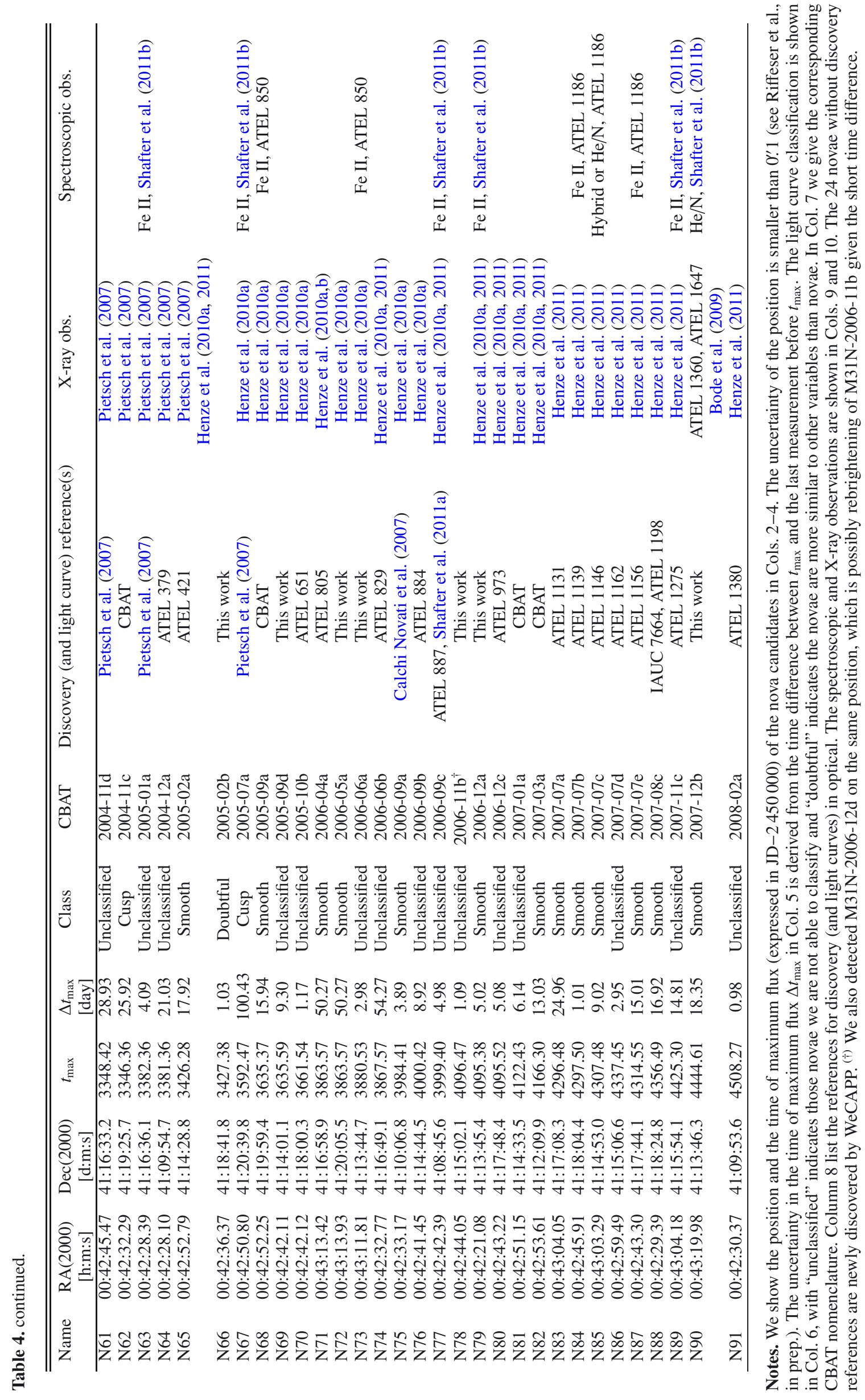

A43, page 7 of 59 


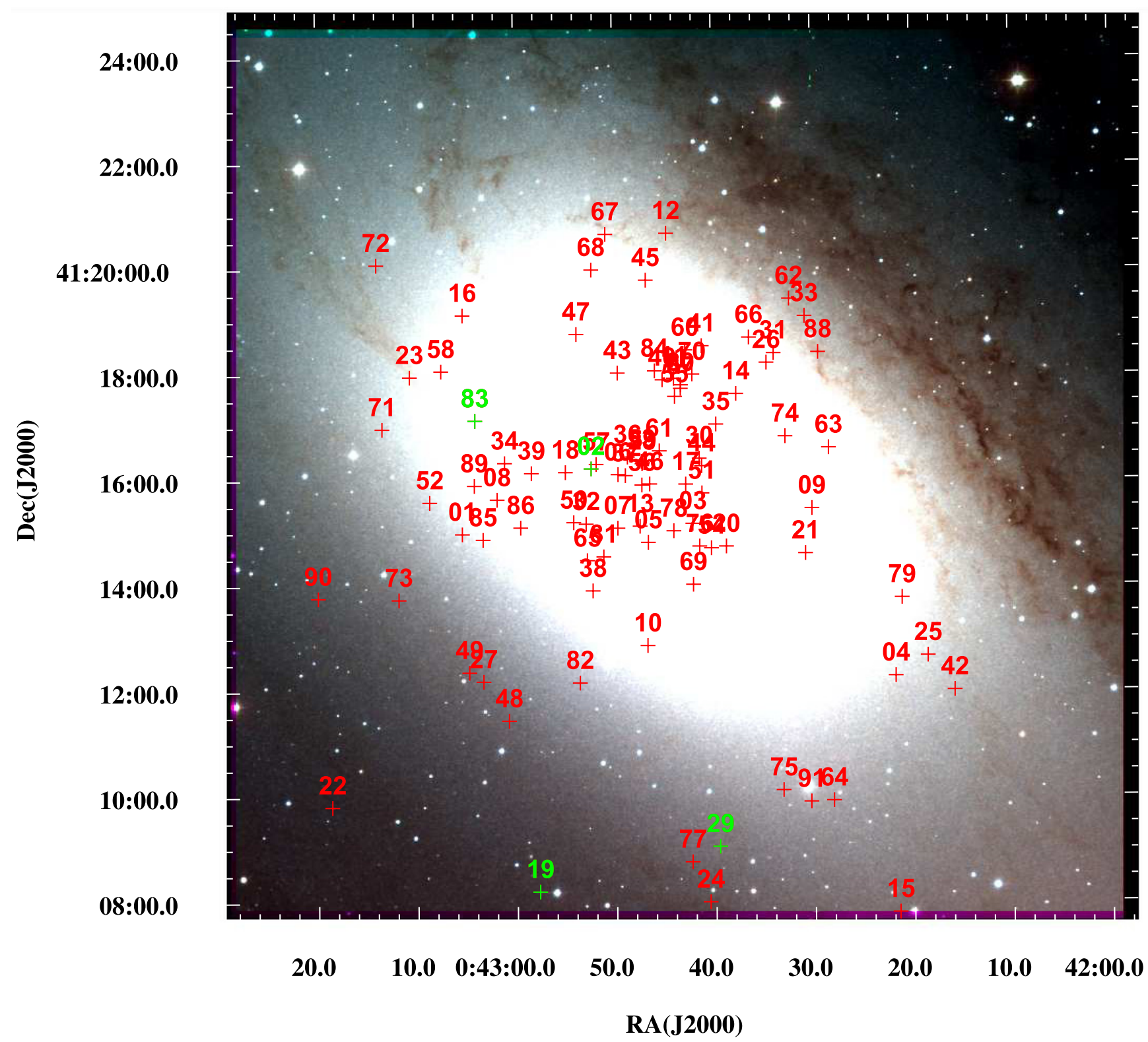

Fig. 4. Distribution of the WeCAPP nova candidates. The recurrent nova candidates (see Sect. 5) are marked in green. The overlaying image is a three-colour-combined image using the observations obtained from Calar Alto observatories in $V, R$, and $I$-band. The image has a size of $17 ! 2 \times 17 ! 2$.

\subsection{O Class}

The O-class light curve follows the S-class light curve, but with the exception that at a given time interval the light curve shows quasi-sinusoidal self-similar oscillations during the course of decline. It has been shown that the white dwarf of the O-class novae is both highly magnetic and massive (Strope et al. 2010). However, these cannot be the only effect leading to oscillation because the nova V1500 Cyg in Strope et al. (2010), which fulfils these requirements but does not show oscillation. There have been many proposals for the mechanism of oscillation, but none of them have been compared to and verified by observation (see Sect. 4 in Strope et al. 2010). The oscillation starts generally around 3 mag below the peak, which indicates that we might have missed the peak in our nova candidates N11 and N28, where the light curves are shown in Fig. 8 and in the Appendix. In Fig. 8 we show the two O-class candidates discovered during our observation campaign.

\subsection{J Class}

The characteristics of J-class novae are the jitters on top of the smooth decline. These jitters are symmetric and sharp-topped flares superposed on the base of S-class light curve, which is the major difference from the O-class novae, while the latter bears oscillations up and down the smooth decline. The jitter usually has variations with amplitude larger than half of a magnitude. Jitters do not occur in the late tail of the light curve and most of them occur within 3 mag below the peak. Strope et al. (2010) further propose two subclasses according to the emergence of the jitters: one subclass has jitters only near the peak, while the other has jitters spread all over the light curve roughly until the nova is 3 mag dimmer than the peak. Among our candidates we found one evident J-class light curve, which belongs to the second subclass of Strope et al. (2010) and is shown in Fig. 9.

It has been reported that there is a gradual increase in the time intervals between two successive jitters 
C.-H. Lee et al.: The Wendelstein Calar Alto Pixellensing Project (WeCAPP): the M 31 nova catalogue

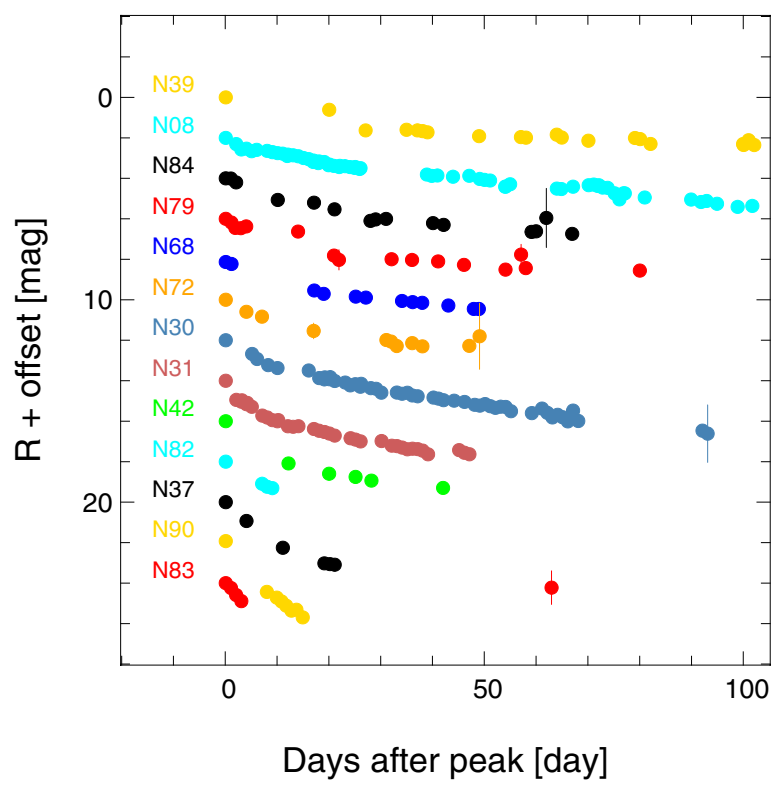

Fig. 5. S Class novae with free $t_{0}$. The single offsets are -15.04 for N08, -3.59 for $\mathrm{N} 30,-2.81$ for $\mathrm{N} 31,2.84$ for N37, -18.02 for N39, -0.88 for $\mathrm{N} 42,-9.89$ for N68, -8.06 for N72, -11.81 for N79, 1.02 for N82, 5.14 for N83, -13.66 for N84, and 5.37 for N90. For most of the data points the error bars are smaller than the symbol of the data points. Here we only show the decline part of the light curve. Full light curves can be found in the Appendix.

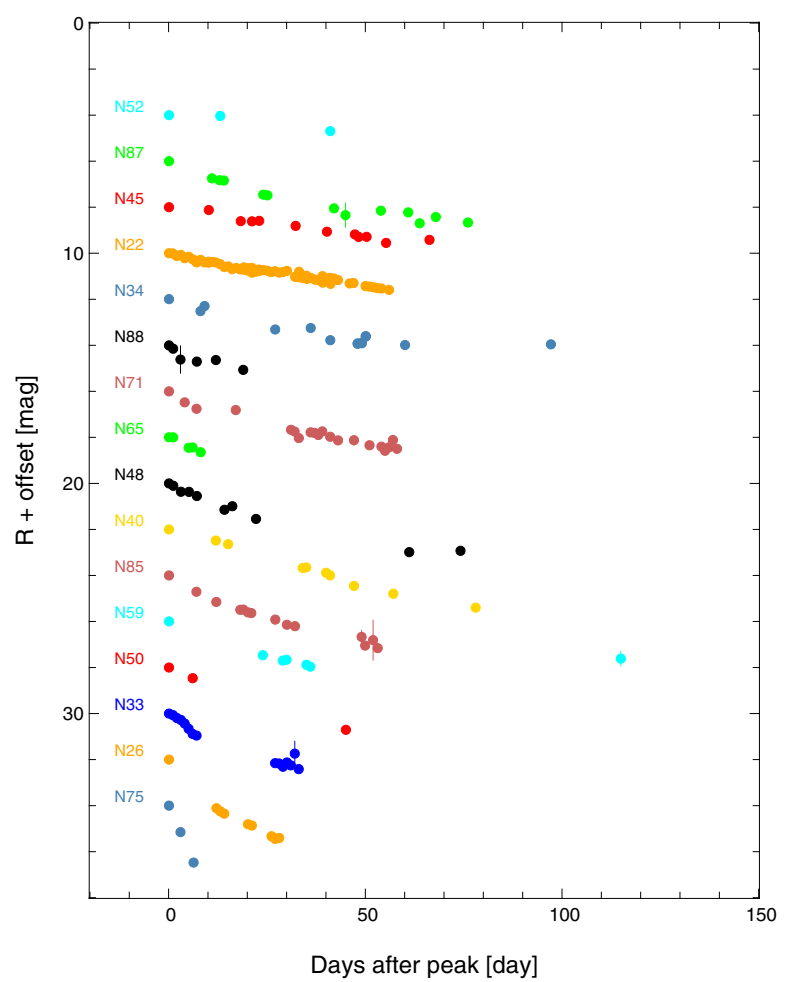

Fig. 6. S Class novae with fixed $t_{0}$. The single offsets are -8.84 for $\mathrm{N} 22$, 15.17 for N26, 12.44 for N33, -6.44 for N34, 4.97 for N40, -10.40 for $\mathrm{N} 45,2.53$ for $\mathrm{N} 48,10.92$ for $\mathrm{N} 50,-13.97$ for $\mathrm{N} 52,8.05$ for $\mathrm{N} 59,-0.22$ for N65, -1.84 for N71, 16.24 for N75, 6.70 for N85, -11.06 for N87, and -5.14 for N88. Here we only show the decline part of the light curve. Full light curves can be found in the Appendix.

(Bianchini et al. 1992; Csák et al. 2005; Pejcha 2009; Tanaka et al. 2011), while Strope et al. (2010) found no distinctive trend
Table 5. Power-law decline fitting for S-class novae.

\begin{tabular}{lcc}
\hline \hline & Free $t_{0}$ & $\alpha$ \\
Name & $t_{0}(\mathrm{JD}-2450000)$ & $\alpha .07 \pm 0.02$ \\
\hline N08 & $1337.9 \pm 0.5$ & $-1.55 \pm 0.01$ \\
N30 & $2257.3 \pm 0.1$ & $-1.29 \pm 0.02$ \\
N31 & $2277.9 \pm 0.2$ & $-3.44 \pm 0.28$ \\
N37 & $2647.4 \pm 1.4$ & $-1.31 \pm 0.04$ \\
N39 & $2776.6 \pm 1.1$ & $-1.21 \pm 0.03$ \\
N42 & $2831.0 \pm 0.2$ & $-1.08 \pm 0.05$ \\
N68 & $3627.7 \pm 0.6$ & $-2.04 \pm 0.16$ \\
N72 & $3845.5 \pm 2.4$ & $-0.89 \pm 0.03$ \\
N79 & $4088.9 \pm 0.4$ & $-2.06 \pm 0.23$ \\
N82 & $4155.1 \pm 1.6$ & $-2.55 \pm 0.51$ \\
N83 & $4288.2 \pm 2.1$ & $-1.05 \pm 0.08$ \\
N84 & $4289.8 \pm 0.9$ & $-3.20 \pm 0.44$ \\
N90 & $4437.3 \pm 1.5$ & \\
\hline & Fixed $t_{0}$ & \\
Name & $t_{0}(J D-2450000)$ & $-4.35 \pm 0.17$ \\
N22 & 1964.3 & $-1.92 \pm 0.04$ \\
N26 & 2145.5 & $-1.22 \pm 0.02$ \\
N33 & 2321.4 & $-1.70 \pm 0.06$ \\
N34 & 2447.5 & $-1.67 \pm 0.03$ \\
N40 & 2798.6 & $-0.97 \pm 0.03$ \\
N45 & 2908.3 & $-0.92 \pm 0.01$ \\
N48 & 2971.4 & $-1.37 \pm 0.04$ \\
N50 & 2985.3 & $-1.43 \pm 0.03$ \\
N52 & 3003.3 & $-1.63 \pm 0.32$ \\
N59 & 3304.4 & $-1.44 \pm 0.10$ \\
N65 & 3408.4 & $-2.92 \pm 0.22$ \\
N71 & 3813.3 & $-2.28 \pm 0.15$ \\
N75 & 3980.5 & $-1.16 \pm 0.05$ \\
N85 & 4298.5 & $-1.48 \pm 0.40$ \\
N87 & 4299.5 & \\
N88 & 4339.6 &
\end{tabular}

using the same data set as Pejcha (2009). We thus tried to search for such trend in our nova candidate N27 and performed a fitting with the following equation:

$\log \left(t_{J}-t_{J-1}\right)=a \log \left(t_{J}-t_{\max }\right)+b$,

where $t_{J}$ is the time of the $J$-th jitter.

The jitters used in the fitting are indicated by the vertical marks in Fig. 9. The time intervals between the successive jitters are shown in Fig. 10. Our best-fitted value is $a=0.64 \pm 0.09$ and $b=0.11 \pm 0.16$. The slope is smaller than the values of DK Lac $(a=0.88)$ and V4745 Sgr $(a=0.79)$ derived by Pejcha (2009) and $a=0.79$ for the 6 novae presented by Tanaka et al. (2011). With only one J-class nova candidate in our catalogue, we can not tell if this is a difference between the novae in M 31 and Galactic novae, or it is simply a variation among individual novae.

\subsection{Other classes}

Besides the above-mentioned classes, three more classes remain in the taxonomy of Strope et al. (2010):

- Flat topped (F) class, which has an extended interval at the peak with near constant brightness.

- Dust dip (D) class where the decline is interrupted by another very steep decline and followed by the recovery to just below the original decline.

- Plateau (P) class that the smooth decline is interrupted by a long-lasting and nearly flat interval, succeeded by the return to the original decline. 

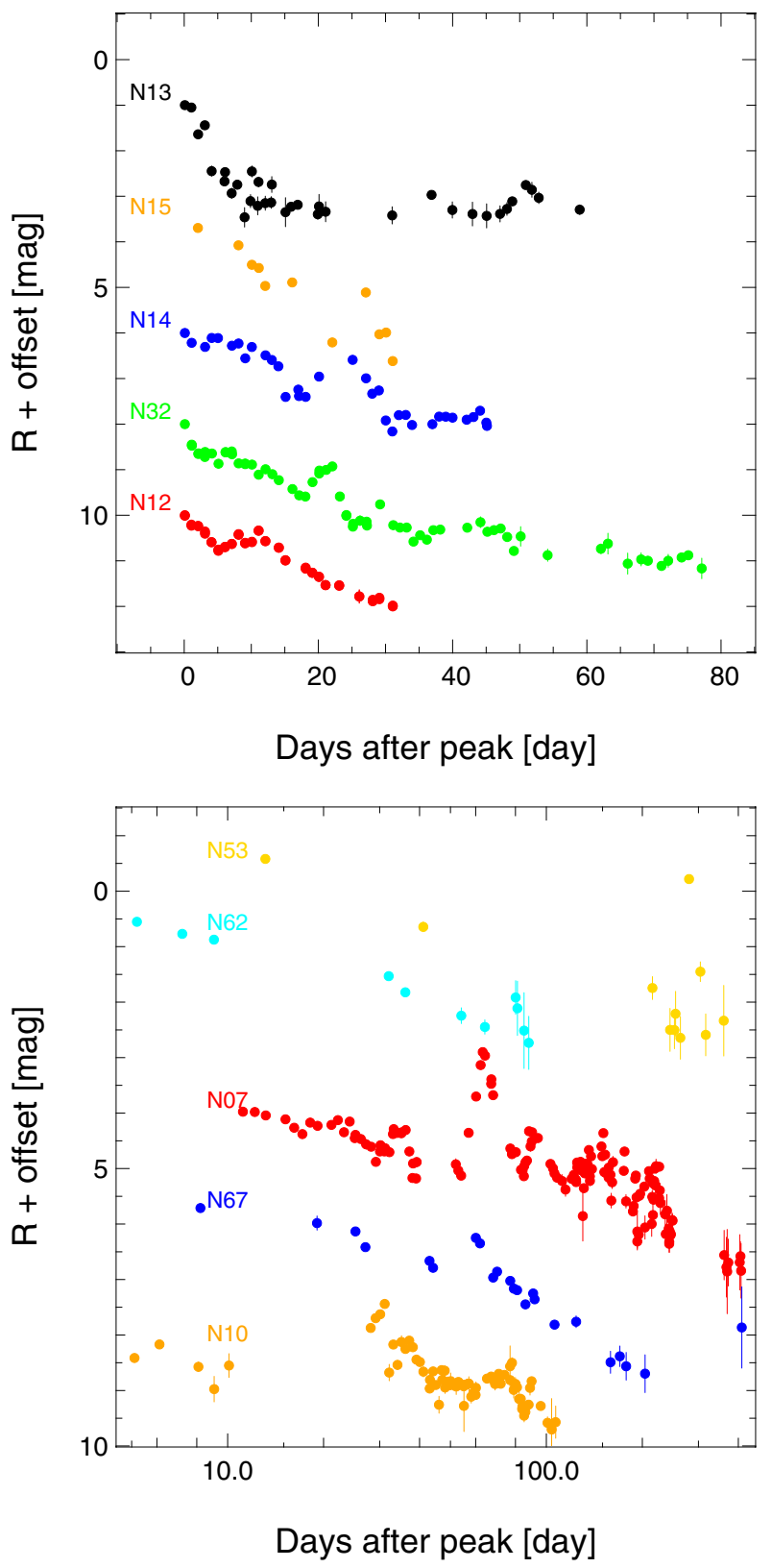

Fig. 7. C Class novae. The offsets applied to the magnitudes are -15.07 for N07, -11.88 for N10, -8.26 for N12, -17.71 for N13, -11.58 for $\mathrm{N} 14,-13.79$ for $\mathrm{N} 15,-9.16$ for $\mathrm{N} 32,-19.19$ for $\mathrm{N} 53,-19.15$ for N62, and -13.04 for N67. Here we only show the decline part of the light curve. Full light curves can be found in the Appendix.

Among our candidates, however, we do not find evident light curves belonging to these classes. This could be partially attributed to the set-up of our observation campaign. For example, the dust dip for the extreme shallow dips in Strope et al. (2010) occur more than one month after the peak, with the dip to be about 6 mag dimmer than the light curve maximum. Such a magnitude variation can hardly be observed in M 31, because it is too faint to be discerned. This implies that we might misclassify the D-class novae into other classes. The non-detection of the P-class novae can be explained by the filter system we used. Hachisu \& Kato (2006) point out that the true plateau from the continuum radiation is best observed in the $y$-band filter. Since we are using the $R$ and $I$-filter, it is possible that the plateau phase

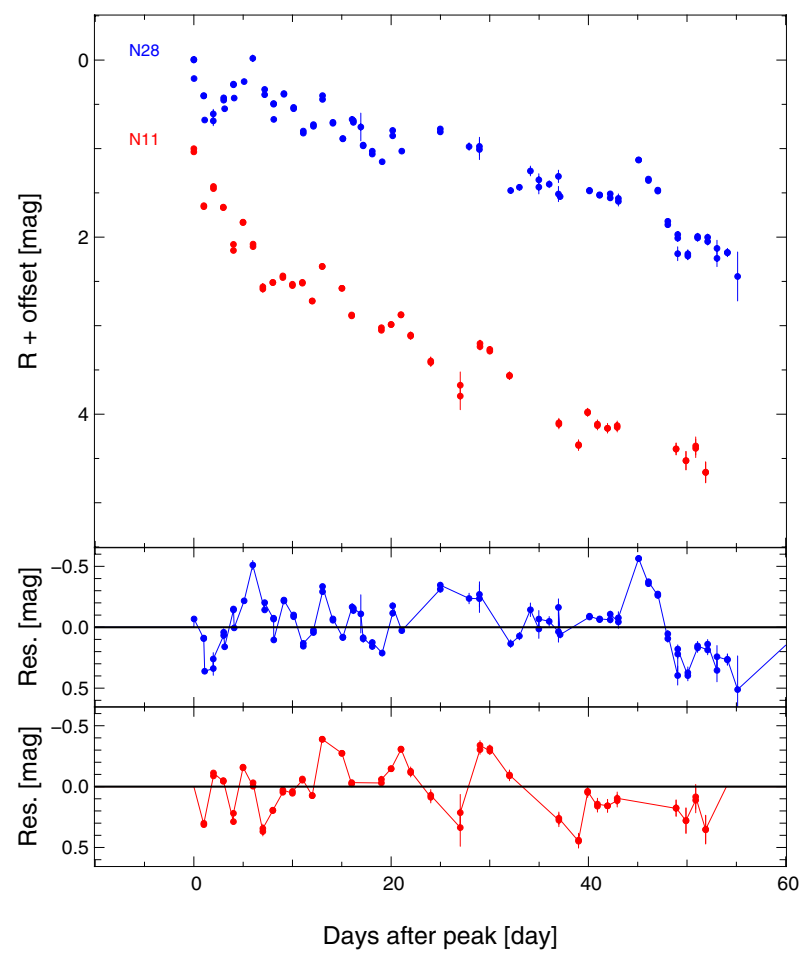

Fig. 8. O Class novae. The offset is -15.77 for N11 and -17.61 for N28. Here we only show the decline part of the light curve. Full light curves can be found in the Appendix.

does not exist in the $R$ and $I$-bands due to the influence of the emission lines during the course of decline.

To summarize, we have classified 42 nova candidates and find $69 \%$ to be S-class, $24 \%$ to be C-class, $5 \%$ to be O-class, and $2 \%$ to be J-class, while Strope et al. (2010) find $38 \%$ to be $\mathrm{S}$-class, $1 \%$ to be C-class, $4 \%$ to be $\mathrm{O}$-class, and $16 \%$ to be $\mathrm{J}$-class in their sample.

\section{Recurrent novae}

Recurrent novae are potential supernovae progenitors (Schaefer 2010). We compared the position of our nova candidates with the catalogue by Pietsch et al. (2007) and Pietsch (2010). We have found four recurrent nova candidates by selecting novae in the literature that are located within 1 arcsec to our nova candidates (see Table 6 and Fig. 11). Among the potential recurrent nova candidates, N29 had three outbursts in 12 years, which would be an unprecedented short period. As pointed out by Henze et al. (2009), the outburst appears earlier in UV and $\mathrm{H} \alpha$ than in the $R$-band, which does not fit very well to the nova scheme. They thus suggest an alternative scheme, where this event could be a dwarf nova in the Milky Way. N19 has four outbursts detected so far. Because of the short time separation between the first two outbursts, Sharov \& Alksnis (1989) have doubted its nova nature and suggested it to be a U Gem type foreground Galactic dwarf nova. However, the spectroscopic observations of the fourth outburst in 2010 (Shafter et al. 2010) have confirmed it as an He/N spectroscopic class nova located in M 31. In addition, Pietsch et al. (2010) also report the SSS turn-on $\sim 15$ days after the first optical detection in 2010.

To test how likely it is that an uncorrelated nova falls into the 1 arcsec area, we performed a test by using the upper-right quarter of our pointing F1, which has the highest M 31 light contribution from the bulge and contains 42 novae. The ratio 
Table 6. Recurrent nova candidates.

\begin{tabular}{|c|c|c|c|c|c|c|c|}
\hline WeCAPP ID & RA & Dec & Name & RA & Dec & Separation & $\Delta_{\mathrm{M} 31}$ \\
\hline N02 (M31N-1997-10f) & $00: 42: 52.35$ & $+41: 16: 13.2$ & M31N-2008-08b & $00: 42: 52.38$ & $+41: 16: 12.9$ & 0.54 & 1.50 \\
\hline N19 (M31N-2001-07b) & $00: 42: 57.75$ & $+41: 08: 12.3$ & M31N-1963-09c & $00: 42: 57.73$ & $+41: 08: 12.4$ & 0.32 & $8: 29$ \\
\hline N19 (M31N-2001-07b) & $00: 42: 57.75$ & $+41: 08: 12.3$ & M31N-1968-09a & $00: 42: 57.71$ & $+41: 08: 11.9$ & 0.72 & $8: 29$ \\
\hline N19 (M31N-2001-07b) & $00: 42: 57.75$ & $+41: 08: 12.3$ & M31N-2010-10e & $00: 42: 57.76$ & $+41: 08: 12.3$ & 0.15 & $8: 29$ \\
\hline N29 (M31N-2001-12b) & $00: 42: 39.59$ & $+41: 09: 02.9$ & M31N-1997-11k & $00: 42: 39.59$ & $+41: 09: 02.9$ & 0.00 & $7 ! 12$ \\
\hline N29 (M31N-2001-12b) & $00: 42: 39.59$ & $+41: 09: 02.9$ & M31N-2009-11b & $00: 42: 39.61$ & $+41: 09: 03.2$ & $0 . \prime 42$ & $7: 12$ \\
\hline N83 (M31N-2007-07a) & 00:43:04.05 & $+41: 17: 08.3$ & M31N-1990-10a & 00:43:04.05 & $+41: 17: 07.5$ & 0.90 & $3: 82$ \\
\hline
\end{tabular}

Notes. We give the WeCAPP name, the positions (also see Fig. 4), the corresponding novae fulfilling the 1'.0 criterion, the separation, and the distance from the centre of M $31\left(\Delta_{\mathrm{M} 31}\right)$.

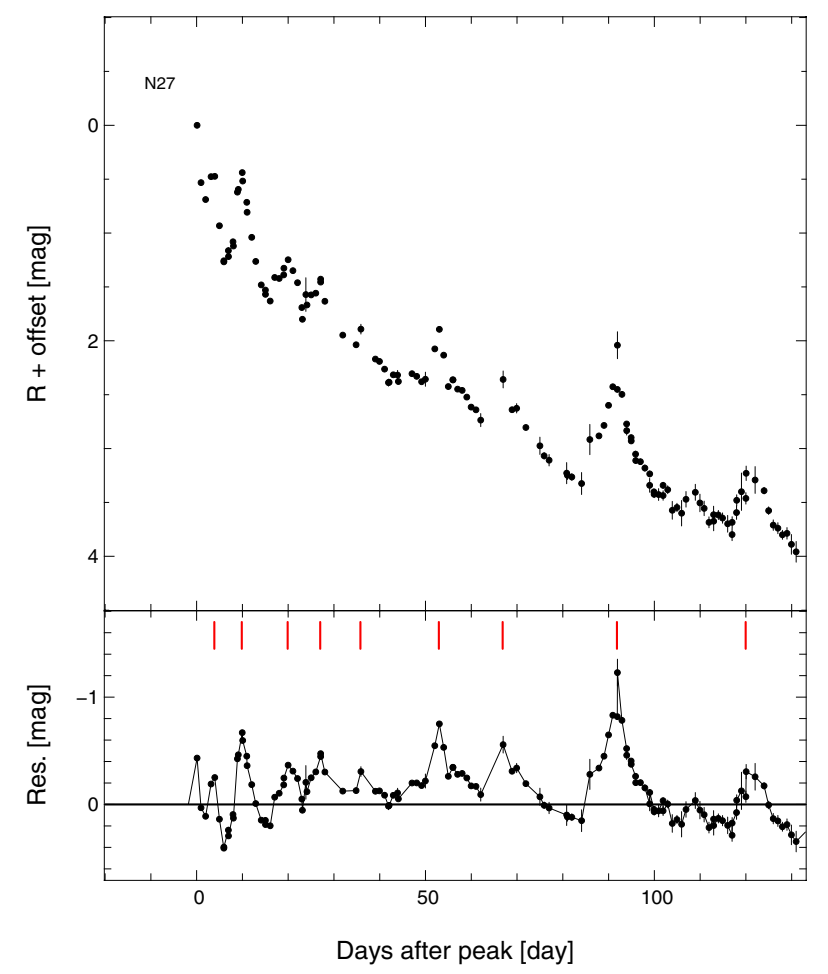

Fig. 9. J Class nova. The single offsets are -16.99 for N27. Here we only show the decline part of the light curve. Full light curves can be found in the Appendix.

of the area occupied by the 1 .' 0 circle of these 42 novae to the total area of this quarter $\left(300 \times 300 \operatorname{arcsec}^{2}\right)$ implies the chance that an uncorrelated nova coincides with an existing nova is low (1.5:1000). As most of the recurrent novae are not found in this quadrant (see Fig. 4 and Table 6), the chance of coincidence is even less for the majority of the recurrent nova candidates. We use stricter selection criteria to search for recurrent novae, thus we have fewer candidates than presented by Pietsch et al. (2007) and Pietsch (2010).

Hachisu et al. (2006) suggest that recurrent novae all bear the plateau light curve. However, in our light curve we did not detect any evident plateau. The main reason is we do not have comprehensive coverage of the light curves. Despite the lack of highly sampled observation, it would be hard to find such plateaus because the light curves in $R$ or $I$ are contaminated by the bright emission lines. Hachisu et al. (2008) thus advocate observations in Strömgren $y$-band (centred at $547 \mathrm{~nm}$ ) since it is designed to cut the strong emission lines in the wide $V$ bandpass filter and can follow the continuum flux more accurately. However, the Strömgren $y$-filter is narrow and requires a longer exposure

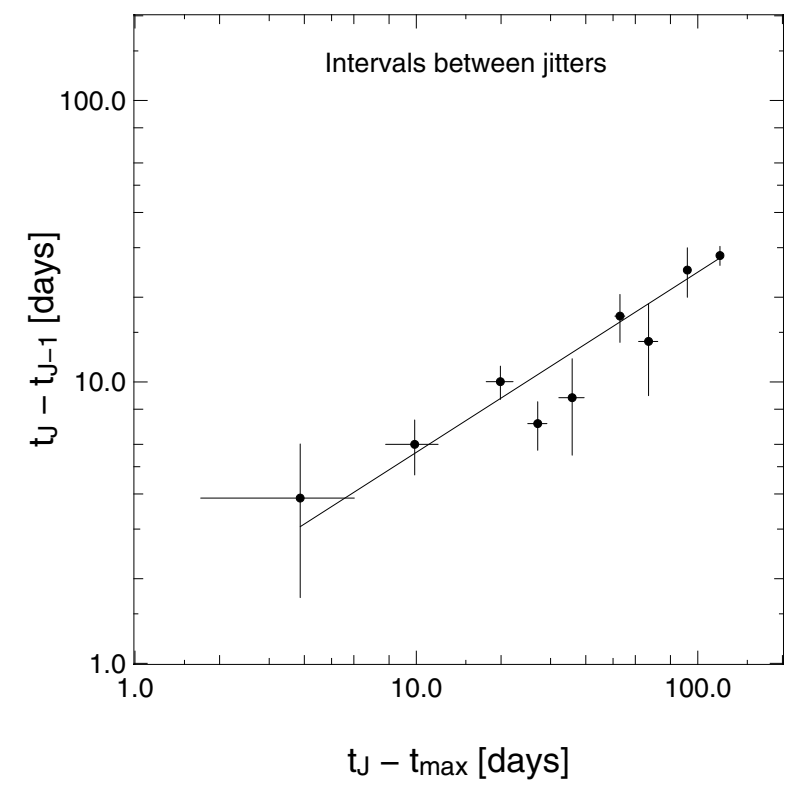

Fig. 10. J-class peak intervals for nova N27, using $R$-band data.
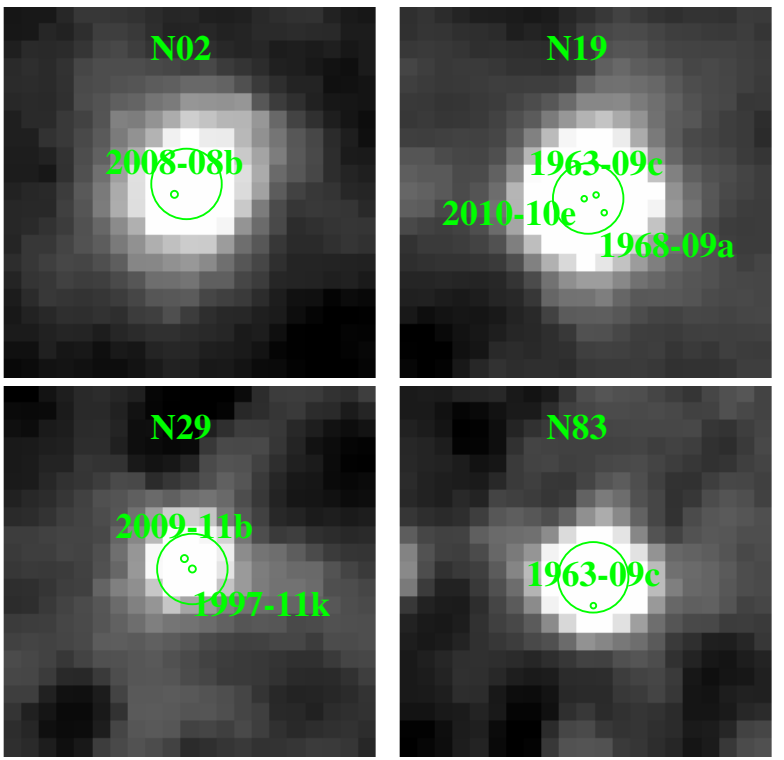

Fig. 11. Position of the recurrent novae. The larger circle in the centre indicates the $1^{\prime \prime} .0$ radius for our selection criteria. The position of potential recurrent nova candidates are marked by the smaller circles.

time, so we use the $I$-filter instead of the Strömgren $y$-filter for the confirmation of microlensing event from achromaticity when the WeCAPP was initiated. 


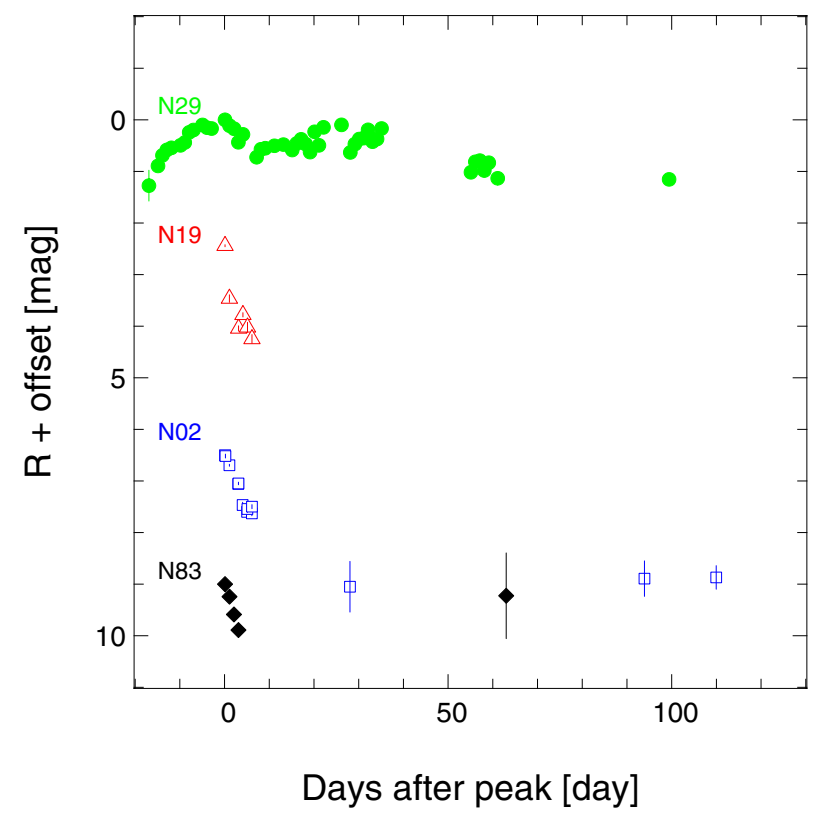

Fig. 12. Recurrent nova candidates' light curves. The single offsets are -11.76 for $\mathrm{N} 02,-16.76$ for N19, -18.86 for N29, and -9.86 for N83. Here we only show the decline part of the light curve. Full light curves can be found in the Appendix.

\section{Rate of decline and the speed class}

In this section we present the rate of decline for our nova sample. Due to the observing gaps before some of the apparent maxima, it is hard to recover the true maximum fluxes during the nova eruption. Nevertheless, the apparent maxima can serve as a lower limit of the true maxima. One can derive upper limits for the $t_{2}$ values (the time required for the nova to faint by two magnitudes) if the apparent maxima are taken as the true maxima. We retrieved the $t_{2}$ values for our sample as follows: we performed a linear fitting on the decline part of each light curve and determined the rate of decline, $\mathrm{d} m / \mathrm{d} t$ (in units of magnitude/day). We then used $\mathrm{d} m / \mathrm{d} t$ to derive the $t_{2}$ value relative to the observed maximum magnitude for all the novae. The result is shown in Fig. 13. Besides the linear fitting, we also applied the universal decline law proposed by Hachisu \& Kato (2006) to retrieve the $t_{2}$ value from the observed magnitude at the apparent maximum. This procedure can only be done for $30 \mathrm{~S}$-class novae, because the fitting routine fails to find a solution for other classes. The result is shown in Fig. 14. The reader should keep in mind, that Figs. 13 or 14 does not give the exact MMRD relation, but serves as an upper limit in the $t_{2}$ and lower limit in the magnitude.

As pointed out by Warner (1989), the value of $\mathrm{d} m / \mathrm{d} t$ is also an indicator of the speed class. We thus use the $\mathrm{d} m / \mathrm{d} t$ values of our sample to derive the frequency of different speed classes of nova in M 31. A comparison of the $\mathrm{d} m / \mathrm{d} t$ distribution in our sample to the novae presented by Darnley et al. (2004) is shown in Fig. 15, the two M 31 samples agree rather well within the statistical errors (shown are only the $\sqrt{n}$ number count errors). The Milky Way data set of Strope et al. (2010) differs significantly by presenting a much higher fraction of very fast novae than the M 31 data. For a fair comparison, one would have to correct the Strope et al. (2010) sample for its severe observational selection effects as being observed from inside the (dusty) Milky Way disk. A more detailed comparison is beyond the scope of this
Linear fit in magnitudes

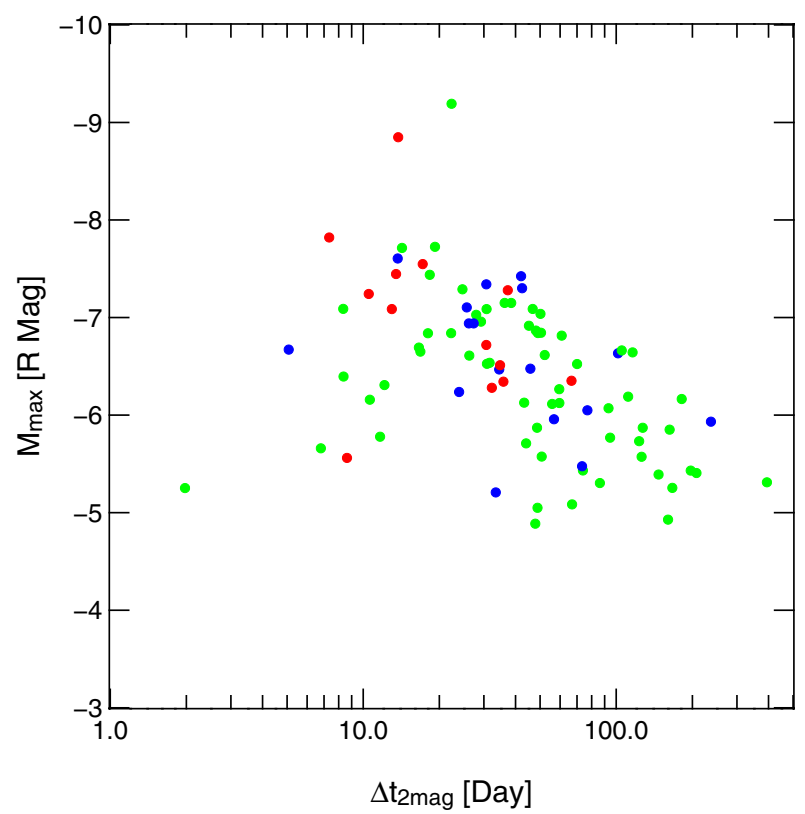

Fig. 13. Distribution of the observed apparent maximum brightness in $R$-band and the fitted $t_{2}$ for all nova candidates. The red and blue points refer to Eq. (3) with $t_{0}$ as free parameter and Eq. (4) with $t_{0}$ as fixed parameter, respectively. The green points are novae that belong to other classes. The $t_{2}$ value is derived from the $\mathrm{d} m / \mathrm{d} t$ and the observed apparent maximum in the light curves. See the main text for a detailed description.

Fit from universal decline law

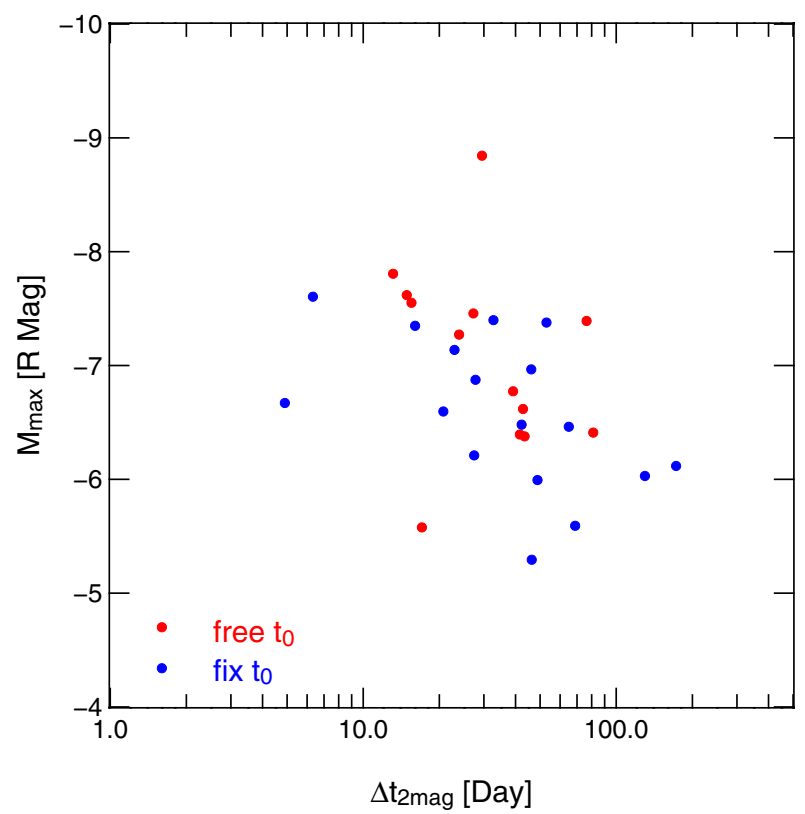

Fig. 14. Distribution of the observed apparent maximum and the fitted $t_{2}$ for S-class novae. The $t_{2}$ value is derived from the universal decline law (Hachisu \& Kato 2006) and the observed apparent maximum in the light curves. See the main text for detailed description. The red and blue points refer to Eq. (3) with $t_{0}$ as a free parameter and Eq. (4) with $t_{0}$ as a fixed parameter, respectively.

paper as it requires detailed modelling of the distribution of stellar and dust population of both galaxies. 
Table 7. Speed class of nova according to Warner (1989).

\begin{tabular}{|c|c|c|c|c|c|}
\hline \multirow[t]{2}{*}{ Speed class } & \multirow{2}{*}{$\begin{array}{c}t_{2} \\
{[\text { day] }}\end{array}$} & \multirow{2}{*}{$\begin{array}{c}\mathrm{d} V / \mathrm{d} t \\
{[\mathrm{mag} / \mathrm{day}]}\end{array}$} & \multicolumn{2}{|c|}{ M 31 sample } & \multirow{2}{*}{$\begin{array}{c}\text { MW sample } \\
\text { Strope et al. (2010) }\end{array}$} \\
\hline & & & this work & Darnley et al. (2004) & \\
\hline Very fast & $\leq 10$ & $\geq 0.2$ & 8 & 1 & 35 \\
\hline Fast & $11-25$ & $0.18-0.08$ & 18 & 3 & 27 \\
\hline Moderately fast & $26-80$ & $0.07-0.025$ & 46 & 11 & 23 \\
\hline Slow & $81-150$ & $0.024-0.013$ & 11 & 2 & 7 \\
\hline Very slow & $151-250$ & $0.013-0.008$ & 7 & 3 & 3 \\
\hline & & $\leq 0.08$ & 1 & 0 & 2 \\
\hline
\end{tabular}

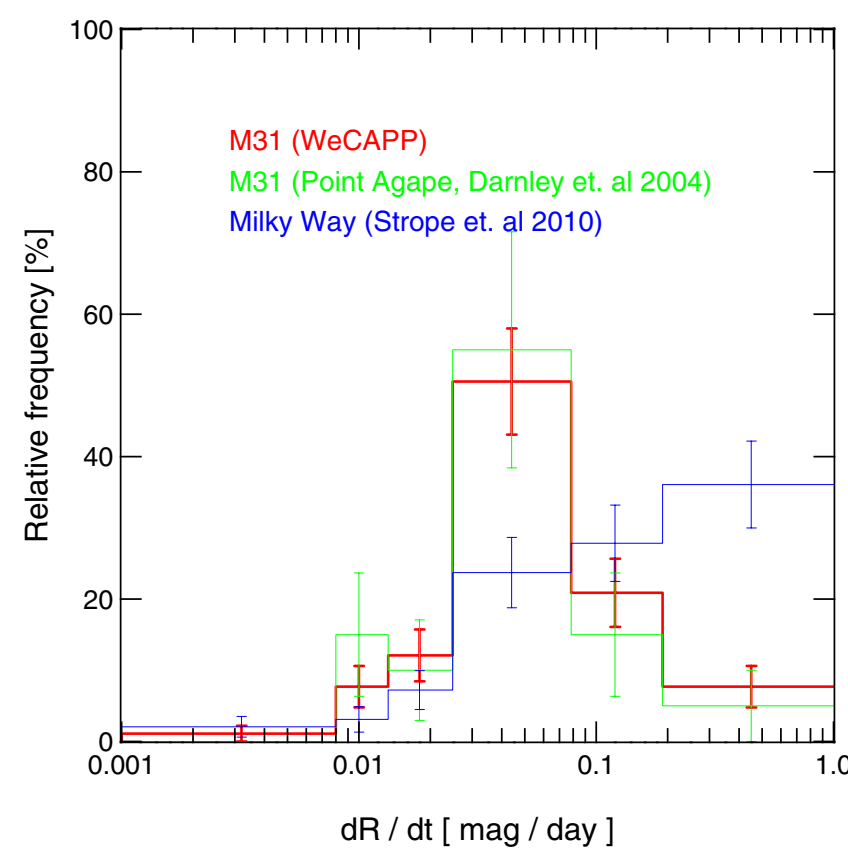

Fig. 15. The distribution of the speed class of novae in M 31 (see Table 7, for definition see Warner 1989). The red line is derived from our sample and the green line is from the M 31 novae presented by Darnley et al. (2004). The blue line represents the Milky Way novae by Strope et al. (2010).

\section{Conclusion and outlook}

We have presented the position, outburst time, and the maximum brightness of the 91 nova candidates discovered during the time span of the WeCAPP project. Light-curve classifications under the taxonomic scheme of Strope et al. (2010) were shown, and the full $R$ and $I$-band light curves of each individual nova during the outburst are also presented in the Appendix.

In this work we successfully applied a scheme developed for a Milky Way nova sample that, because of observational selection effects, is certainly dominated by the galactic disk, to a nova sample of a different host galaxy that is mostly observed towards the bulge of this host. The differences in member ratios for the subclasses as defined by Strope et al. (2010) between the Milky Way and our M 31 WeCAPP sample probably reflect different observational selection effects to some extent, but can potentially lead to further conclusions on the differences between stellar populations in M 31 and the Milky Way, once the selection effects are proper accounted for.

We provide the full light curve data of the novae on request, as well as the postage stamps of the reduced, stacked, or difference-imaging frames.

Part of this catalogue has been used to find the X-ray counterpart by Pietsch et al. $(2005,2007)$ to show that super soft X-ray sources (SSS) in M 31 are mostly constituted by the novae during eruption. The turn on and turn off of the SSS phase provide us the information of the ejected and accreted mass onto the surface of the white dwarf.

Besides the X-ray monitoring campaign, there is also a survey of M 31 novae in infrared using Spitzer Space Telescope (Shafter et al. 2011a), which indicates a correlation between the dust formation timescales and the nova speed class. Such studies would not be possible without the speed class determined by the optical observations. Ground-based optical surveys, such as PTF (Law et al. 2009; Rau et al. 2009), PanSTARRS (Kaiser et al. 2002), and LSST (Tyson 2002), will continue to play an important role in the regime of multi-wavelength novae observation and help us to gain insight of the underlying physical mechanism of novae.

Acknowledgements. We are grateful for the comments from the anonymous referee. We thank Sara Bühler and Silona Wilke for their contributions in observations. This work was supported by the DFG cluster of excellence 'Origin and Structure of the Universe' (www.universe-cluster.de).

\section{References}

Alard, C., \& Lupton, R. H. 1998, ApJ, 503, 325

An, J. H., Evans, N. W., Hewett, P., et al. 2004, MNRAS, 351, 1071

Ansari, R., Aurière, M., Baillon, P., et al. 2004, A\&A, 421, 509

Arp, H. C. 1956, AJ, 61, 15

Bianchini, A., Friedjung, M., \& Brinkmann, W. 1992, A\&A, 257, 599

Bode, M. F., Darnley, M. J., Shafter, A. W., et al. 2009, ApJ, 705, 1056

Calchi Novati, S., Covone, G., de Paolis, F., et al. 2007, A\&A, 469, 115

Calchi Novati, S., Dall'Ora, M., Gould, A., et al. 2010, ApJ, 717, 987

Ciardullo, R., Ford, H. C., Neill, J. D., Jacoby, G. H., \& Shafter, A. W. 1987, ApJ, 318, 520

Ciardullo, R., Shafter, A. W., Ford, H. C., et al. 1990, ApJ, 356, 472

Csák, B., Kiss, L. L., Retter, A., Jacob, A., \& Kaspi, S. 2005, A\&A, 429, 599

Darnley, M. J., Bode, M. F., Kerins, E., et al. 2004, MNRAS, 353, 571

Darnley, M. J., Bode, M. F., Kerins, E., et al. 2006, MNRAS, 369, 257

della Valle, M., \& Livio, M. 1995, ApJ, 452, 704

Fliri, J., Riffeser, A., Seitz, S., \& Bender, R. 2006, A\&A, 445, 423

Gössl, C. A., \& Riffeser, A. 2002, A\&A, 381, 1095

Hachisu, I., \& Kato, M. 2006, ApJS, 167, 59

Hachisu, I., \& Kato, M. 2009, ApJ, 694, L103

Hachisu, I., Kato, M., Kiyota, S., et al. 2006, ApJ, 651, L141

Hachisu, I., Kato, M., Kiyota, S., et al. 2008, in RS Ophiuchi (2006) and the Recurrent Nova Phenomenon, ed. A. Evans, M. F. Bode, T. J. O’Brien, \& M. J. Darnley (San Francisco: ASP), ASP Conf. Ser., 401, 206

Henze, M., Pietsch, W., Podigachoski, P., et al. 2009, The Astronomer's Telegram, 2286, 1

Henze, M., Pietsch, W., Haberl, F., et al. 2010a, A\&A, 523, A89

Henze, M., Pietsch, W., Haberl, F., et al. 2010b, Astron. Nachr., 331, 193

Henze, M., Pietsch, W., Haberl, F., et al. 2011, A\&A, 533, A52

Hubble, E. P. 1929, ApJ, 69, 103

José, J., \& Hernanz, M. 2007, J. Phys. G Nucl. Phys., 34, 431

Joshi, Y. C., Pandey, A. K., Narasimha, D., et al. 2004, A\&A, 415, 471

Kaiser, N., Aussel, H., Burke, B. E., et al. 2002, in SPIE Conf. Ser. 4836, ed. J. A. Tyson, \& S. Wolff, SPIE, Bellingham, 154

Kato, T., Nakajima, K., Maehara, H., \& Kiyota, S. 2009, Variable Star Bulletin, 49, 1

Law, N. M., Kulkarni, S. R., Dekany, R. G., et al. 2009, PASP, 121, 1395

Livio, M. 1992, ApJ, 393, 516 
Lynch, D. K., Woodward, C. E., Gehrz, R., et al. 2008, AJ, 136, 1815 Mclaughlin, D. B. 1945, PASP, 57, 69

Orio, M. 2006, ApJ, 643, 844

Payne-Gaposchkin, C. 1964, The galactic novae, ed. C. H. P. Gaposchkin, New York, Dover

Pejcha, O. 2009, ApJ, 701, L119

Pietsch, W. 2010, Astron. Nachr., 331, 187

Pietsch, W., Fliri, J., Freyberg, M. J., et al. 2005, A\&A, 442, 879

Pietsch, W., Haberl, F., Sala, G., et al. 2007, A\&A, 465, 375

Pietsch, W., Henze, M., Haberl, F., \& Burwitz, V. 2010, The Astronomer's Telegram, 3038, 1

Rau, A., Kulkarni, S. R., Law, N. M., et al. 2009, PASP, 121, 1334

Rector, T. A., Jacoby, G. H., Corbett, D. L., \& Denham, M. 1999a, BAAS, 31, 1420

Rector, T. A., Jacoby, G. H., Corbett, D. L., Denham, M., \& RBSE Nova Search Team 1999b, in Am. Astron. Soc. Meeting Abstracts, $195,36.08$

Riffeser, A., Fliri, J., Gössl, C. A., et al. 2001, A\&A, 379, 362

Riffeser, A., Fliri, J., Bender, R., Seitz, S., \& Gössl, C. A. 2003, ApJ, 599, L17

Riffeser, A., Seitz, S., \& Bender, R. 2008, ApJ, 684, 1093

Rosino, L. 1964, Ann. Astrophys., 27, 498

Rosino, L. 1973, A\&AS, 9, 347

Rosino, L., Capaccioli, M., D’Onofrio, M., \& della Valle, M. 1989, AJ, 97, 83

Schaefer, B. E. 2010, ApJS, 187, 275
Shafter, A. W. 1997, ApJ, 487, 226

Shafter, A. W., \& Irby, B. K. 2001, ApJ, 563, 749

Shafter, A. W., Bode, M. F., Darnley, M. J., Ciardullo, R., \& Misselt, K. A. 2010, The Astronomer's Telegram, 3006, 1

Shafter, A. W., Bode, M. F., Darnley, M. J., et al. 2011a, ApJ, 727, 50

Shafter, A. W., Darnley, M. J., Hornoch, K., et al. 2011b, ApJ, 734, 12

Shara, M. M. 1981, ApJ, 243, 926

Sharov, A. S., \& Alksnis, A. K. 1989, SvA Lett., 15, 382

Sharov, A. S., \& Alksnis, A. 1991, Ap\&SS, 180, 273

Sharov, A. S., Alksnis, A., Zharova, A. V., \& Shokin, Y. A. 2000, Astron. Lett., 26,433

Simon, V., Hornoch, K., Kušnirák, P., Šarounová, L., \& Wolf, M. 2005, in The Astrophysics of Cataclysmic Variables and Related Objects, ed. J.-M. Hameury, \& J.-P. Lasota (San Francisco: ASP), ASP Conf. Ser., 330, 449

Strope, R. J., Schaefer, B. E., \& Henden, A. A. 2010, AJ, 140, 34

Tanaka, J., Nogami, D., Fujii, M., Ayani, K., \& Kato, T. 2011, PASJ, 63, 159

Tomaney, A. B., \& Shafter, A. W. 1992, ApJS, 81, 683

Tyson, J. A. 2002, in SPIE Conf. Ser. 4836, ed. J. A. Tyson, \& S. Wolff, SPIE, Bellingham, 10

Warner, B. 1989, in Classical Novae, ed. M. F. Bode, \& A. Evans (Cambridge: Cambridge Univ. Press), 1

Williams, R. E. 1992, AJ, 104, 725

Zwicky, F. 1936, PASP, 48, 191 
C.-H. Lee et al.: The Wendelstein Calar Alto Pixellensing Project (WeCAPP): the M 31 nova catalogue

\section{Appendix A: WeCAPP nova candidate light curves}

This Appendix contains light curves and postage stamps ( $R$-band in upper row and $I$-band in lower row) for all the candidates.
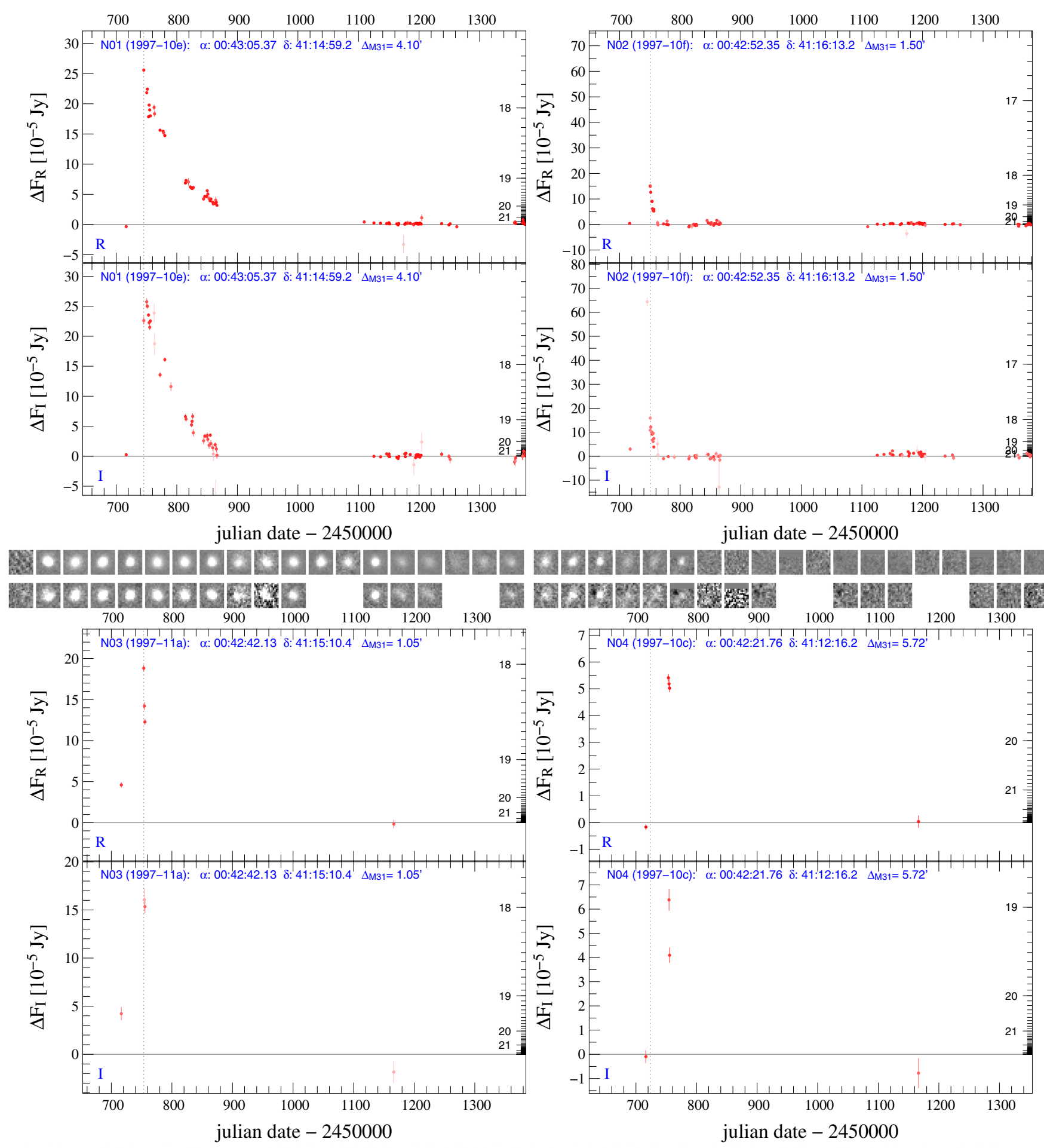

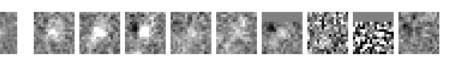
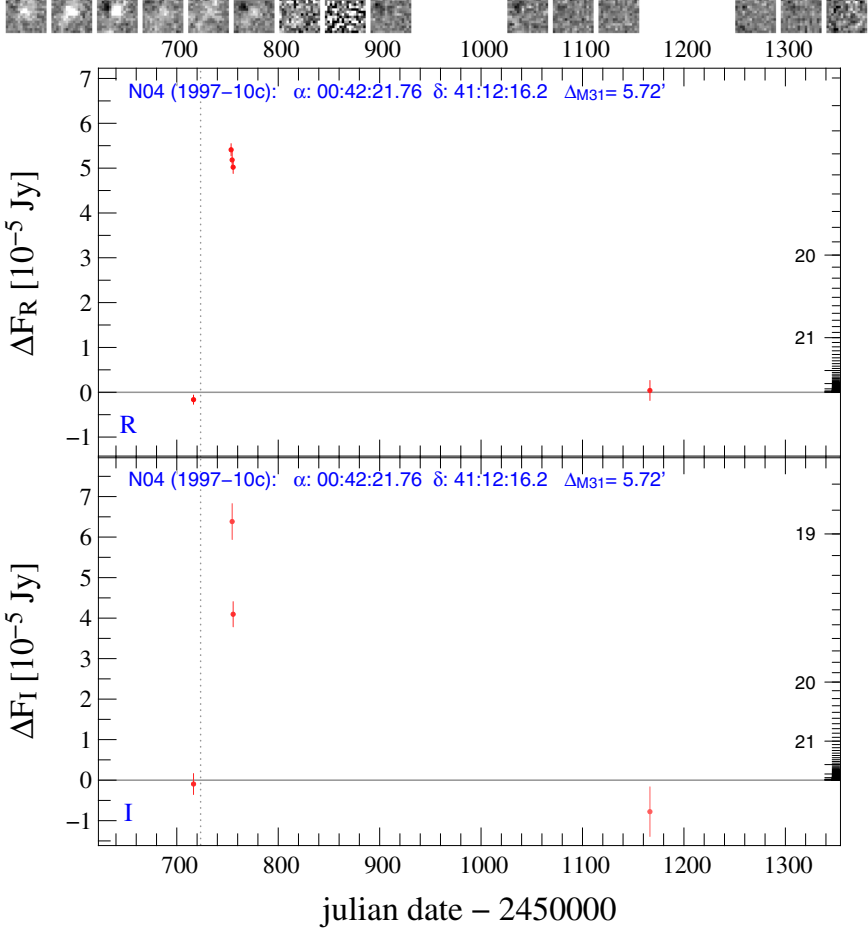

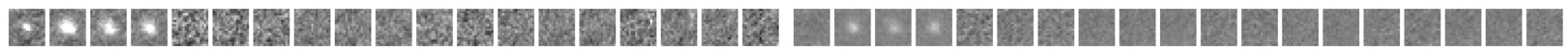

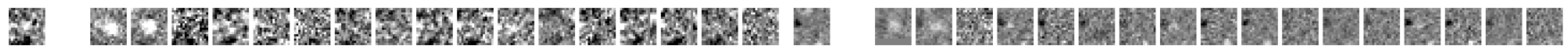

Fig. A.1. Light curve of WeCAPP nova candidates N01, N02, N03 and N04. The numbers on the right of the light curves are the corresponding $R$ and $I$-band magnitudes. 
A\&A 537, A43 (2012)
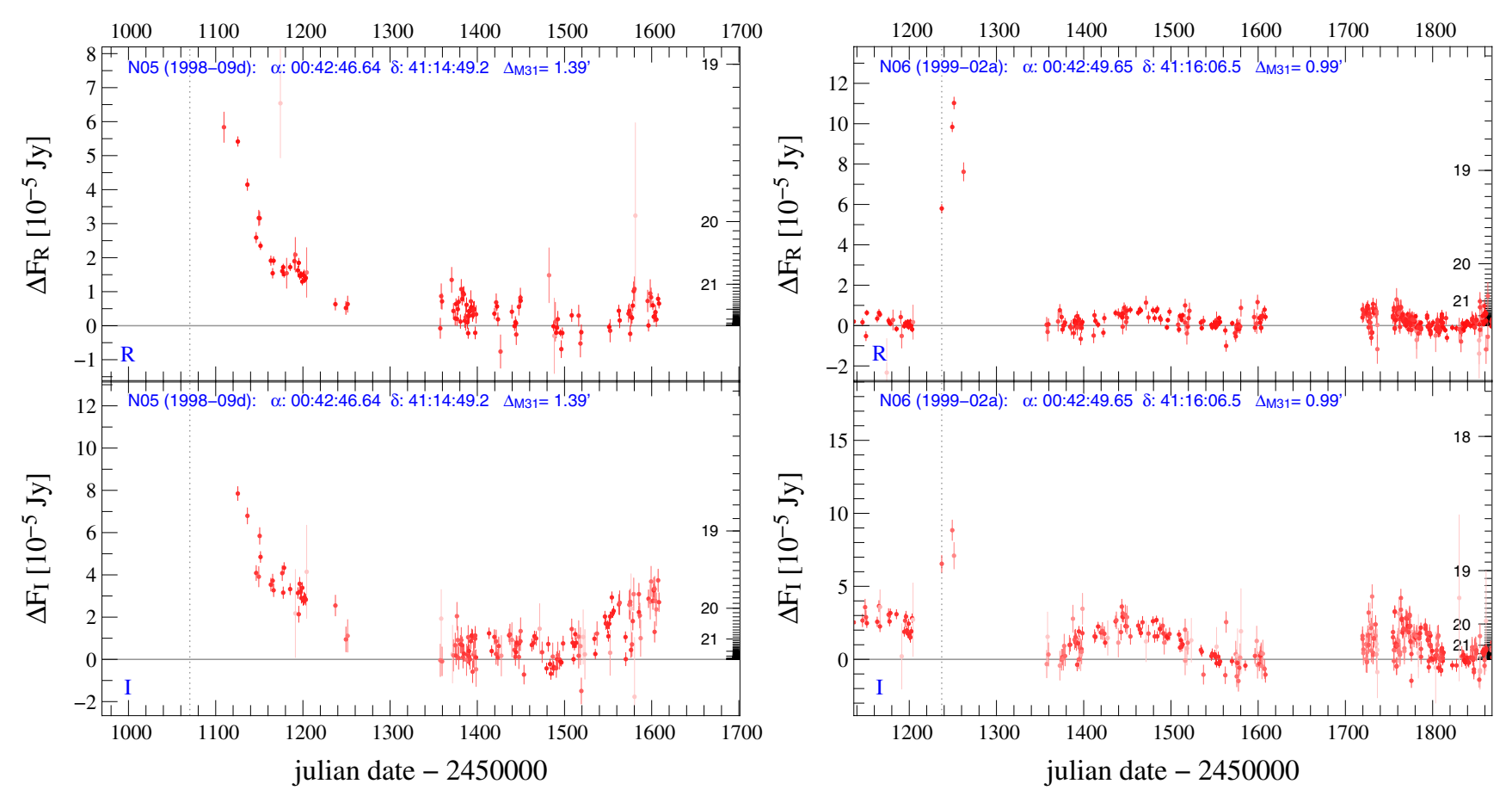

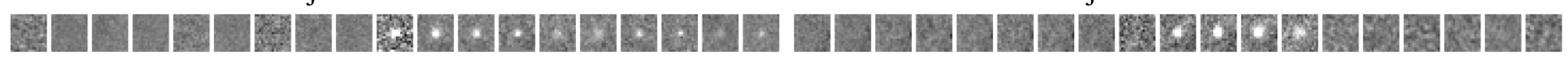

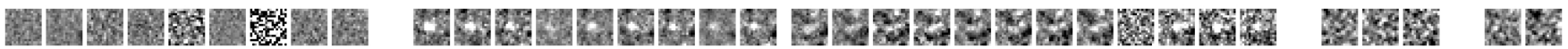
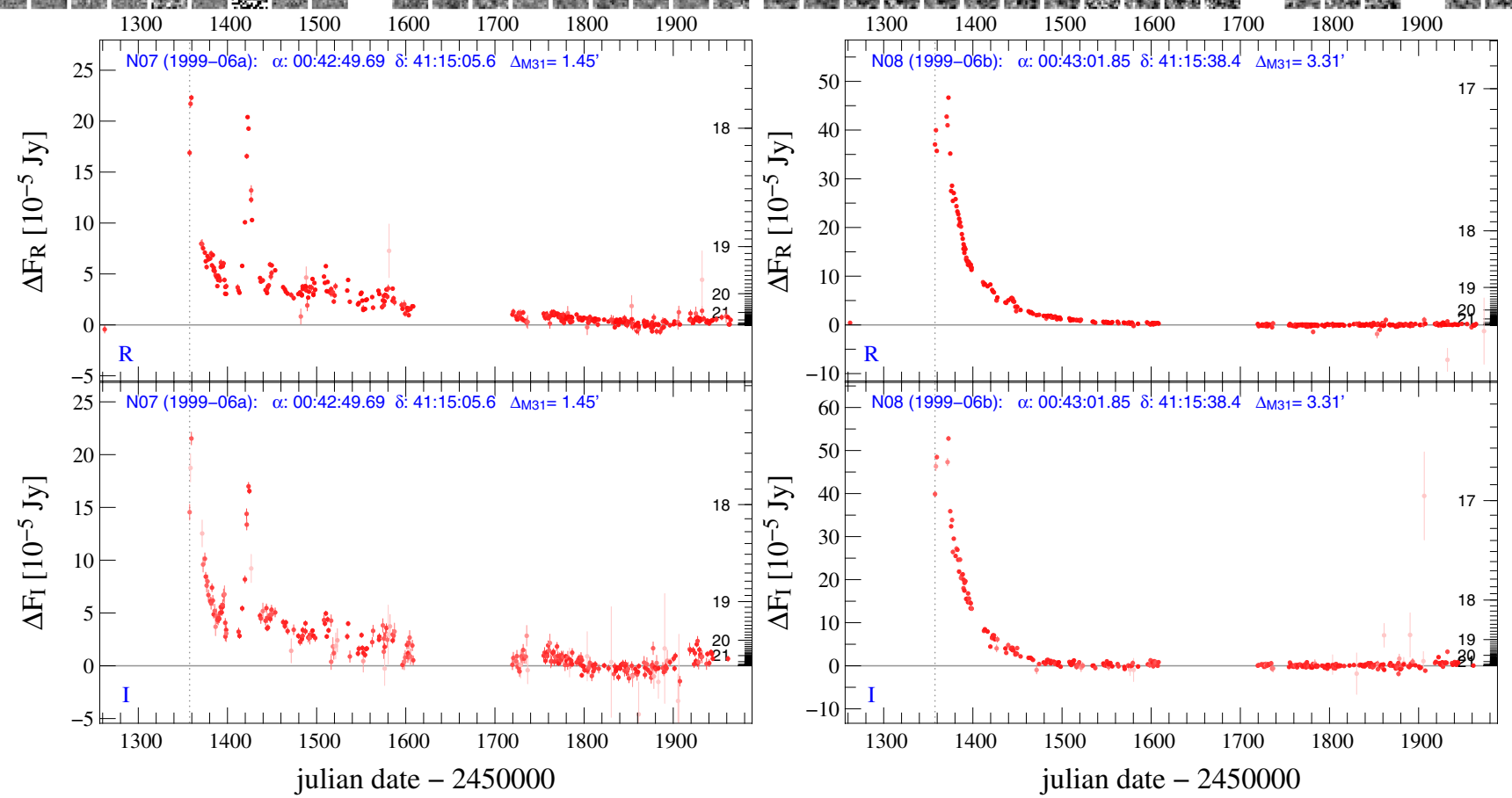

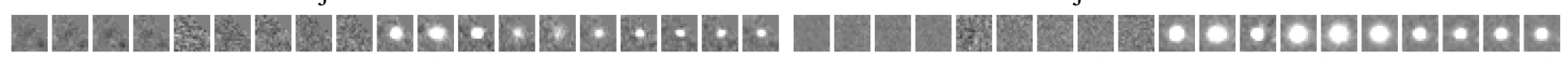

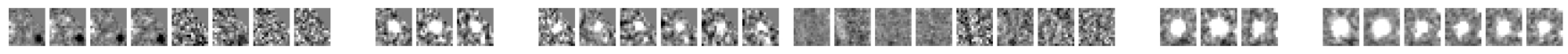

Fig. A.2. Light curve of WeCAPP nova candidates N05, N06, N07 and N08. 
C.-H. Lee et al.: The Wendelstein Calar Alto Pixellensing Project (WeCAPP): the M 31 nova catalogue
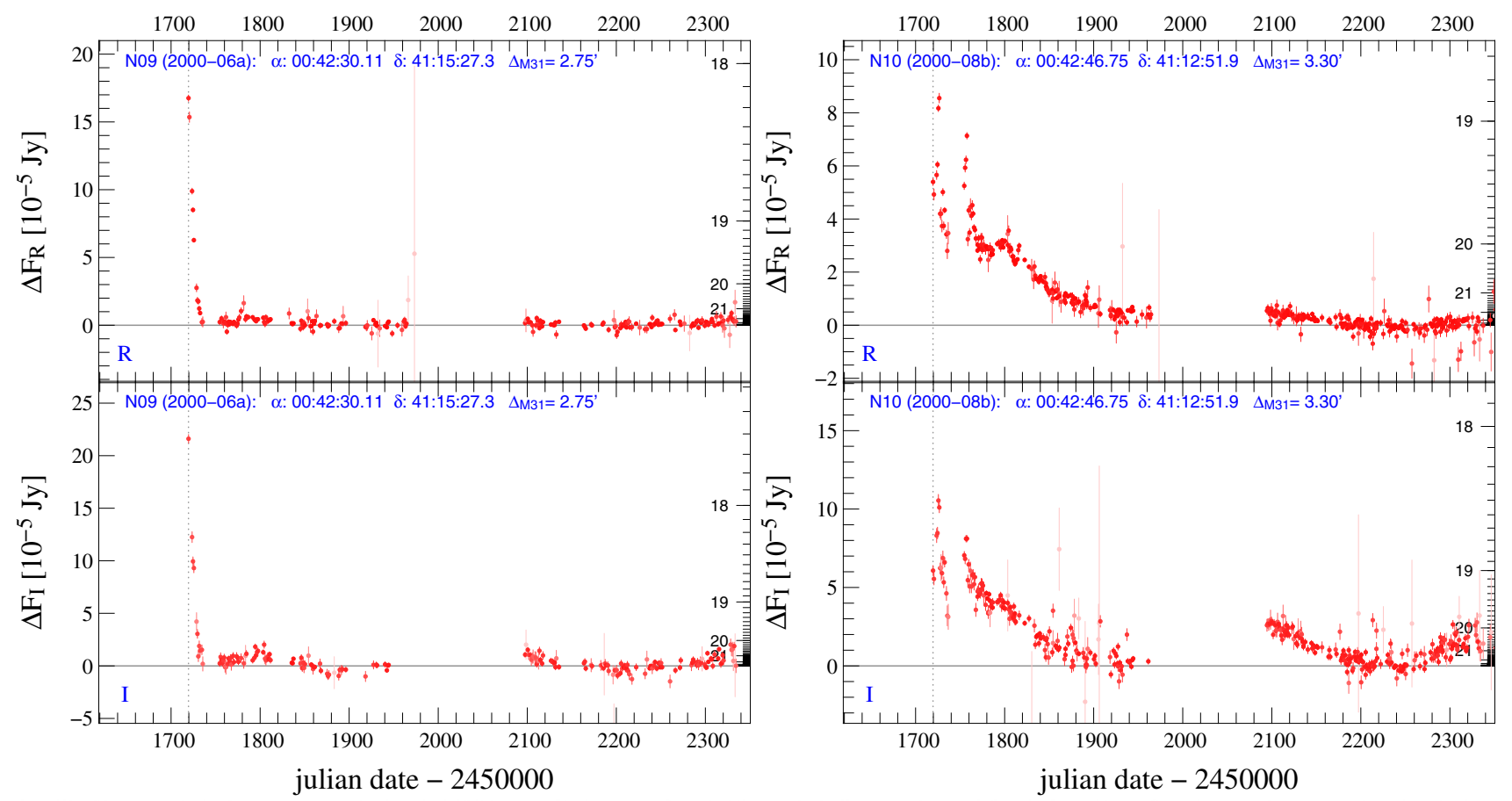

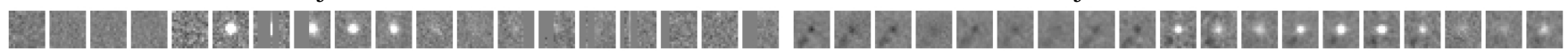
F
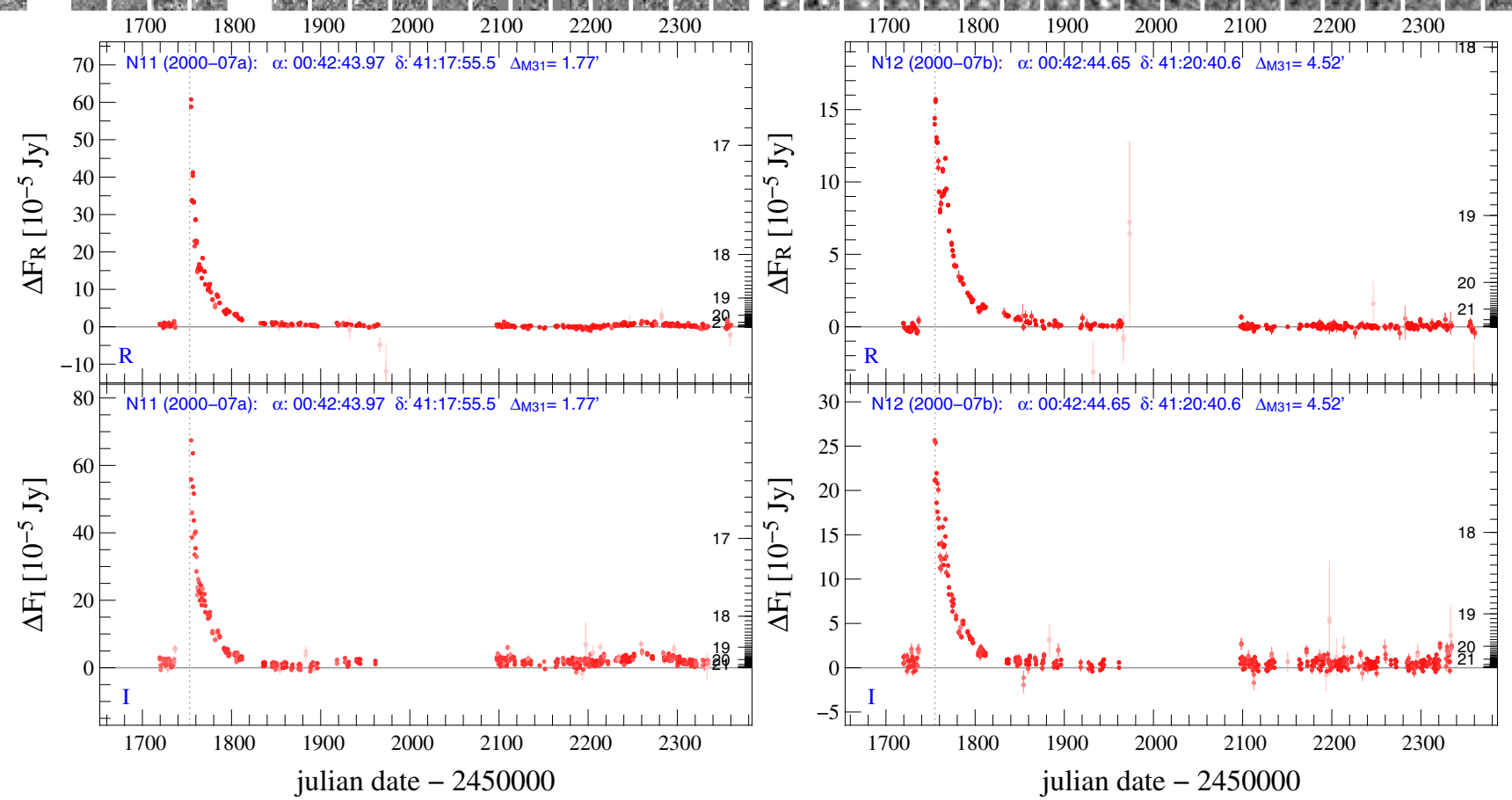

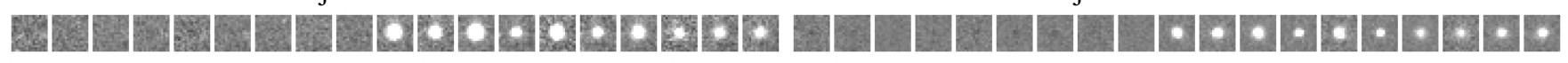
BA

Fig. A.3. Light curve of WeCAPP nova candidates N09, N10, N11 and N12. 
A\&A 537, A43 (2012)
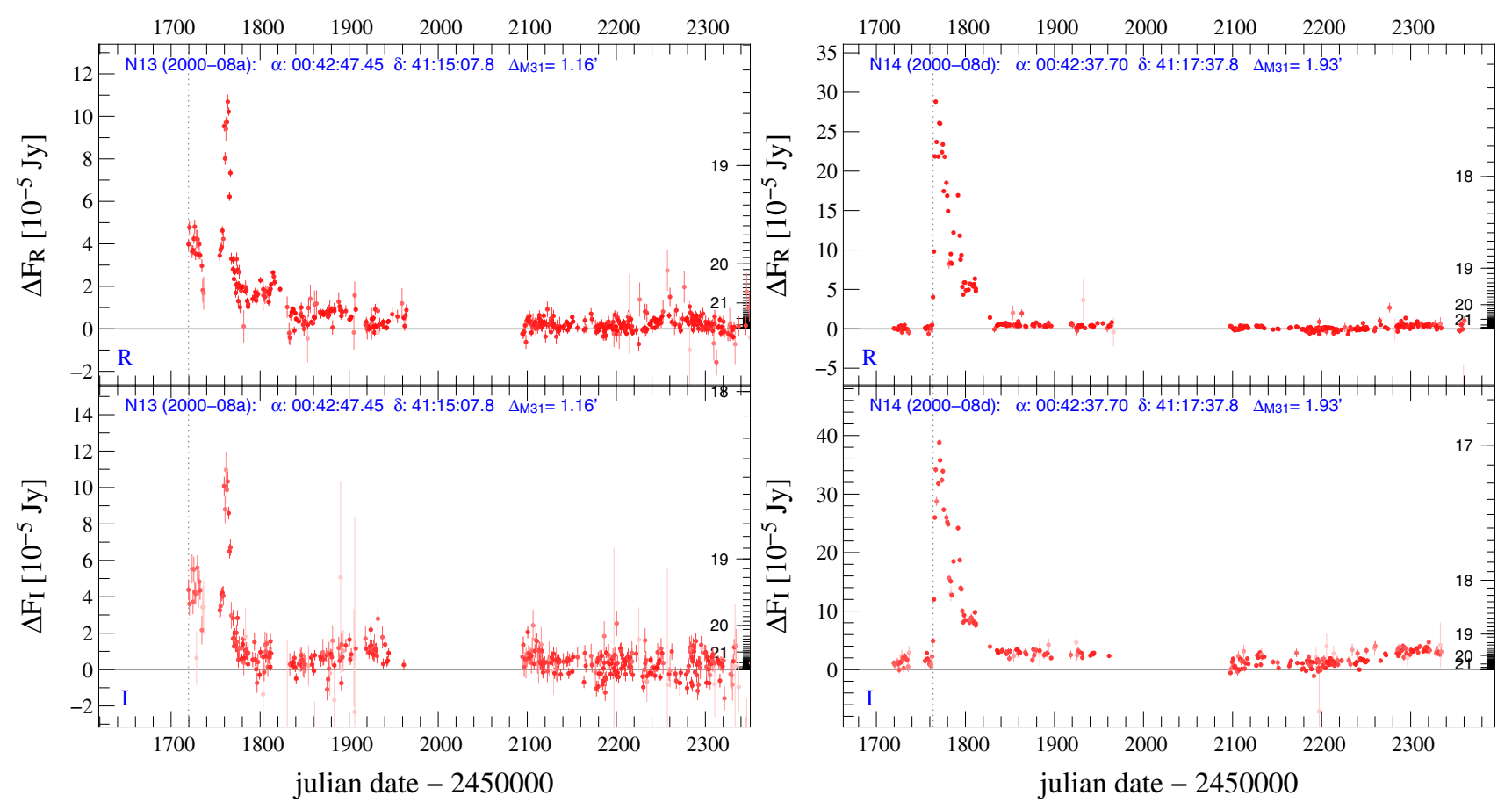

A2

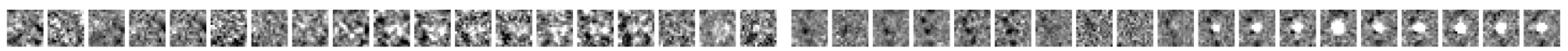
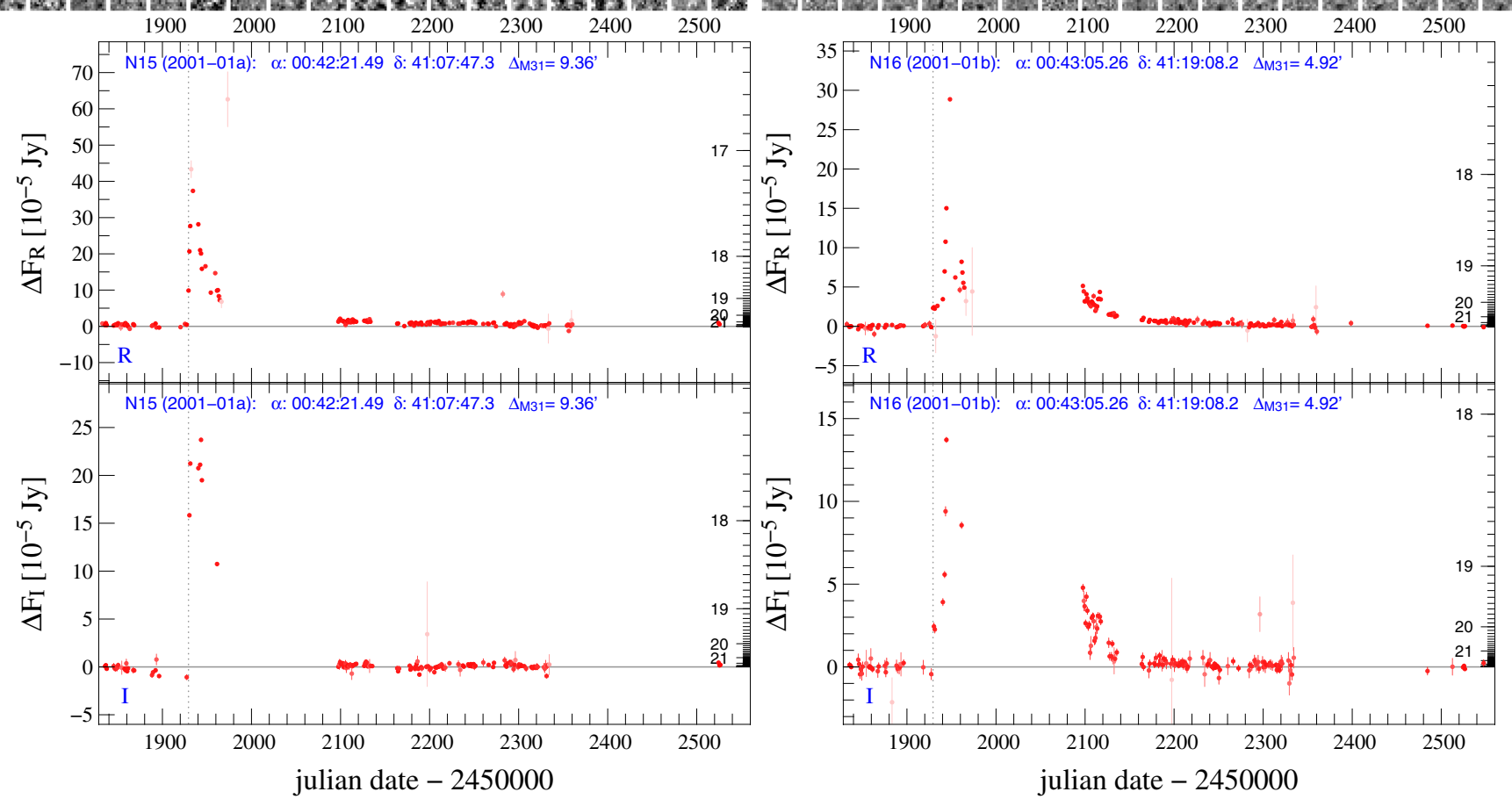

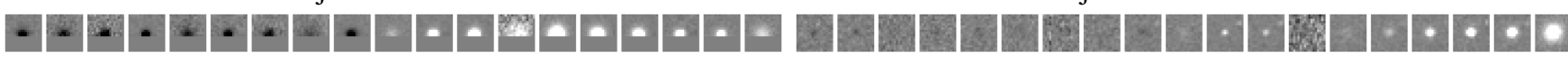

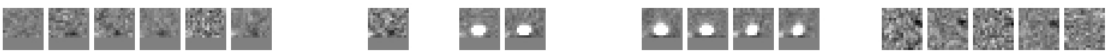

Fig. A.4. Light curve of WeCAPP nova candidates N13, N14, N15 and N16. 
C.-H. Lee et al.: The Wendelstein Calar Alto Pixellensing Project (WeCAPP): the M 31 nova catalogue
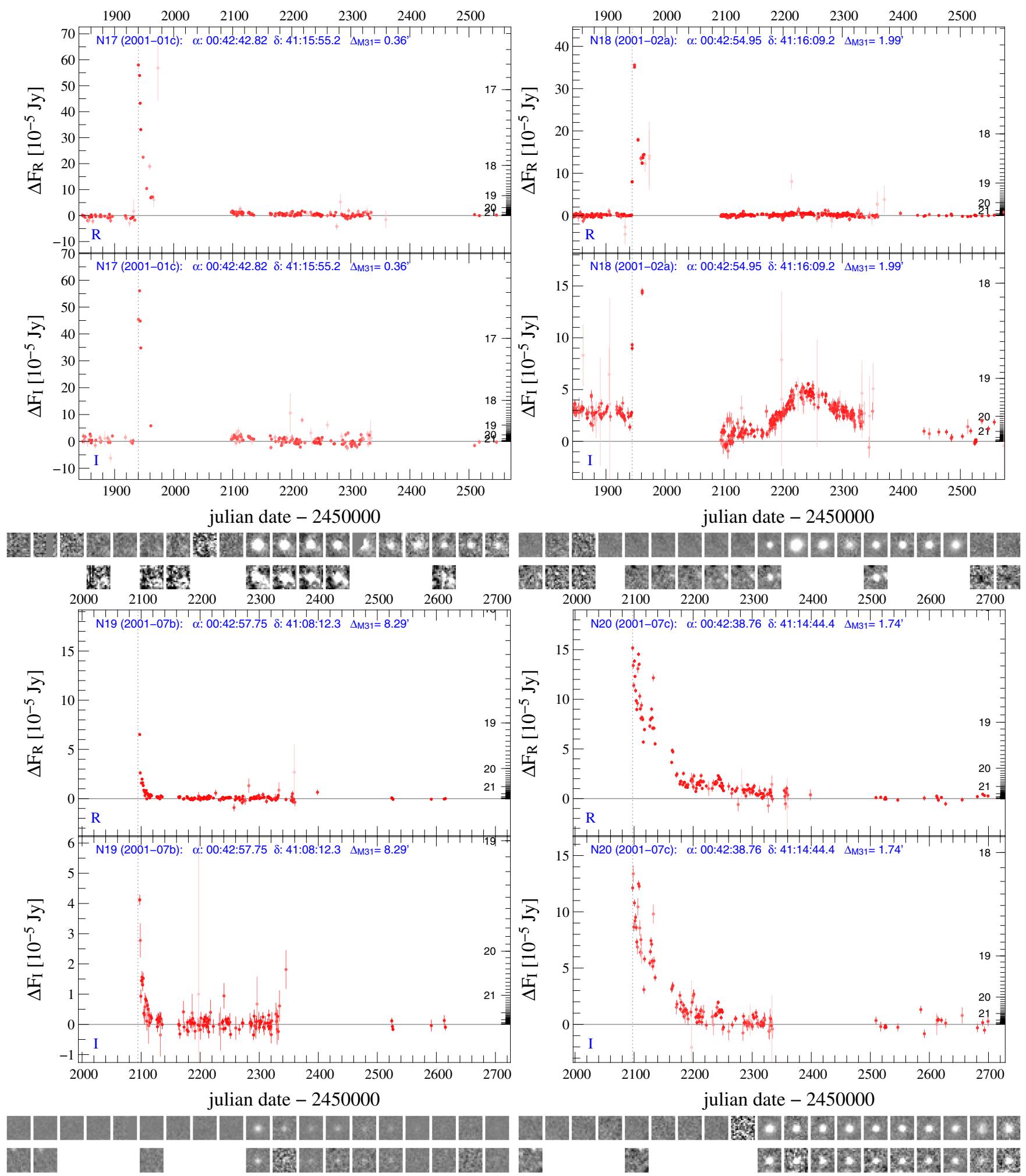

Fig. A.5. Light curve of WeCAPP nova candidates N17, N18, N19 and N20. 

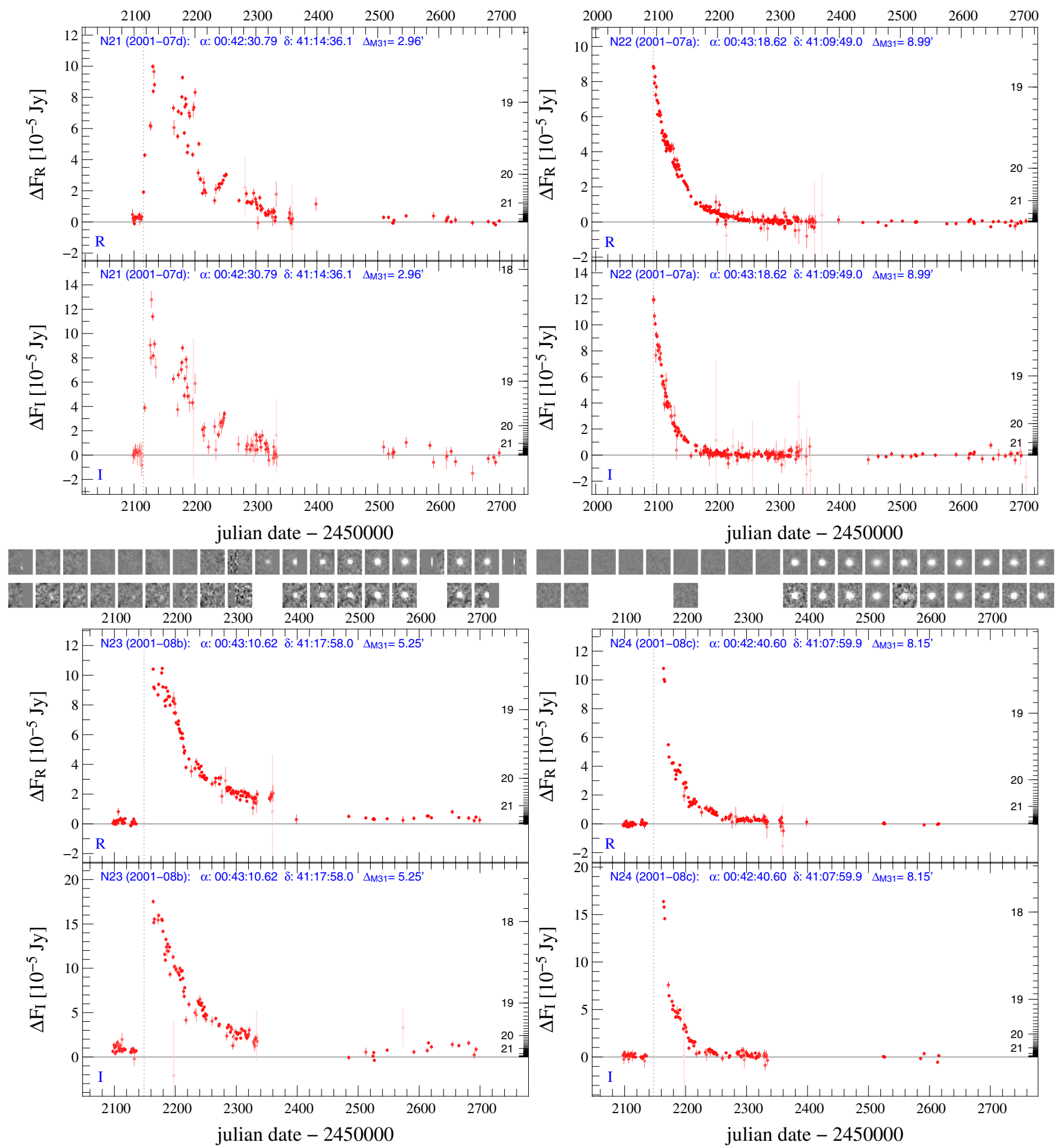

1
1

Fig. A.6. Light curve of WeCAPP nova candidates N21, N22, N23 and N24. 
C.-H. Lee et al.: The Wendelstein Calar Alto Pixellensing Project (WeCAPP): the M 31 nova catalogue
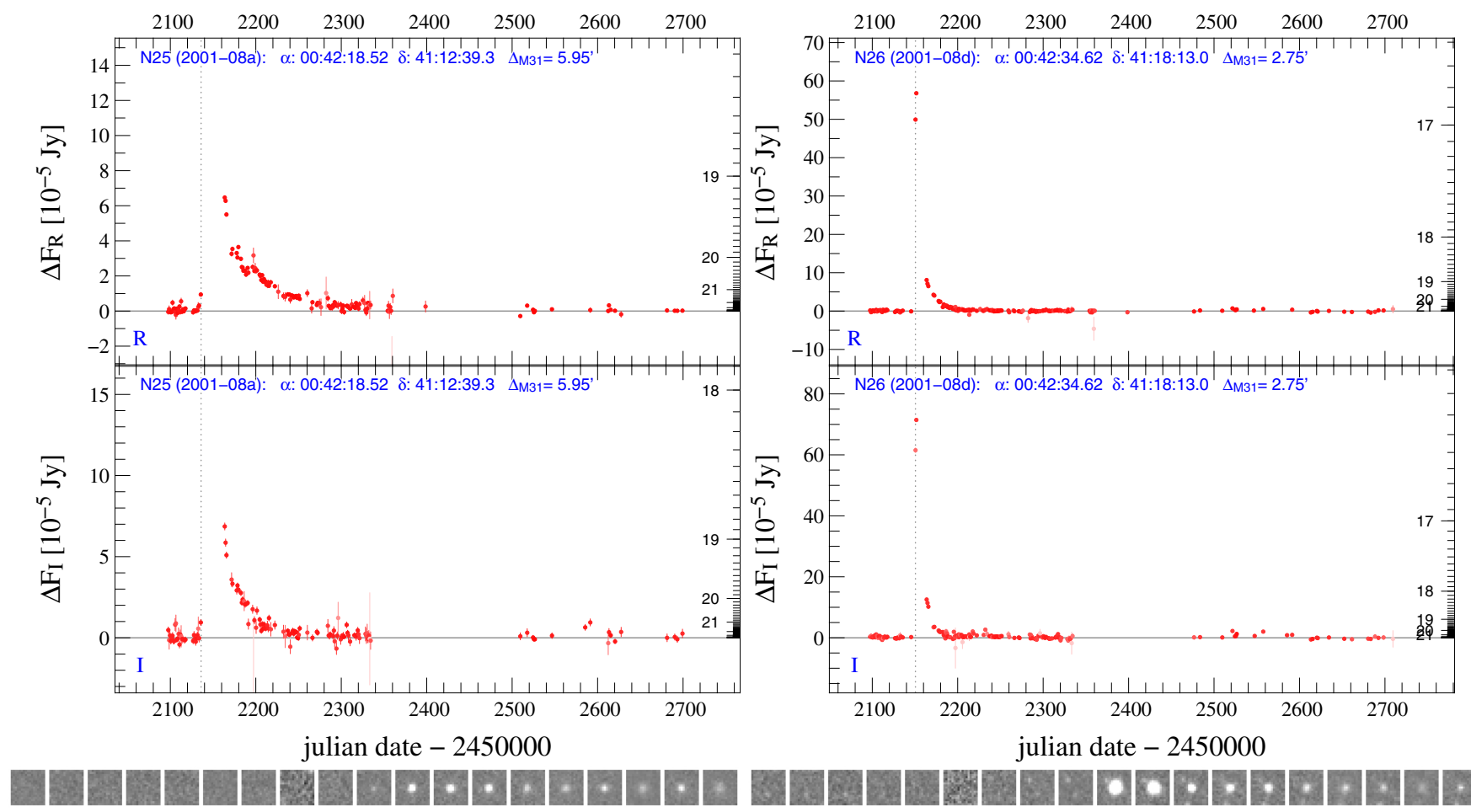

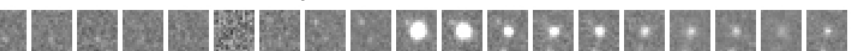
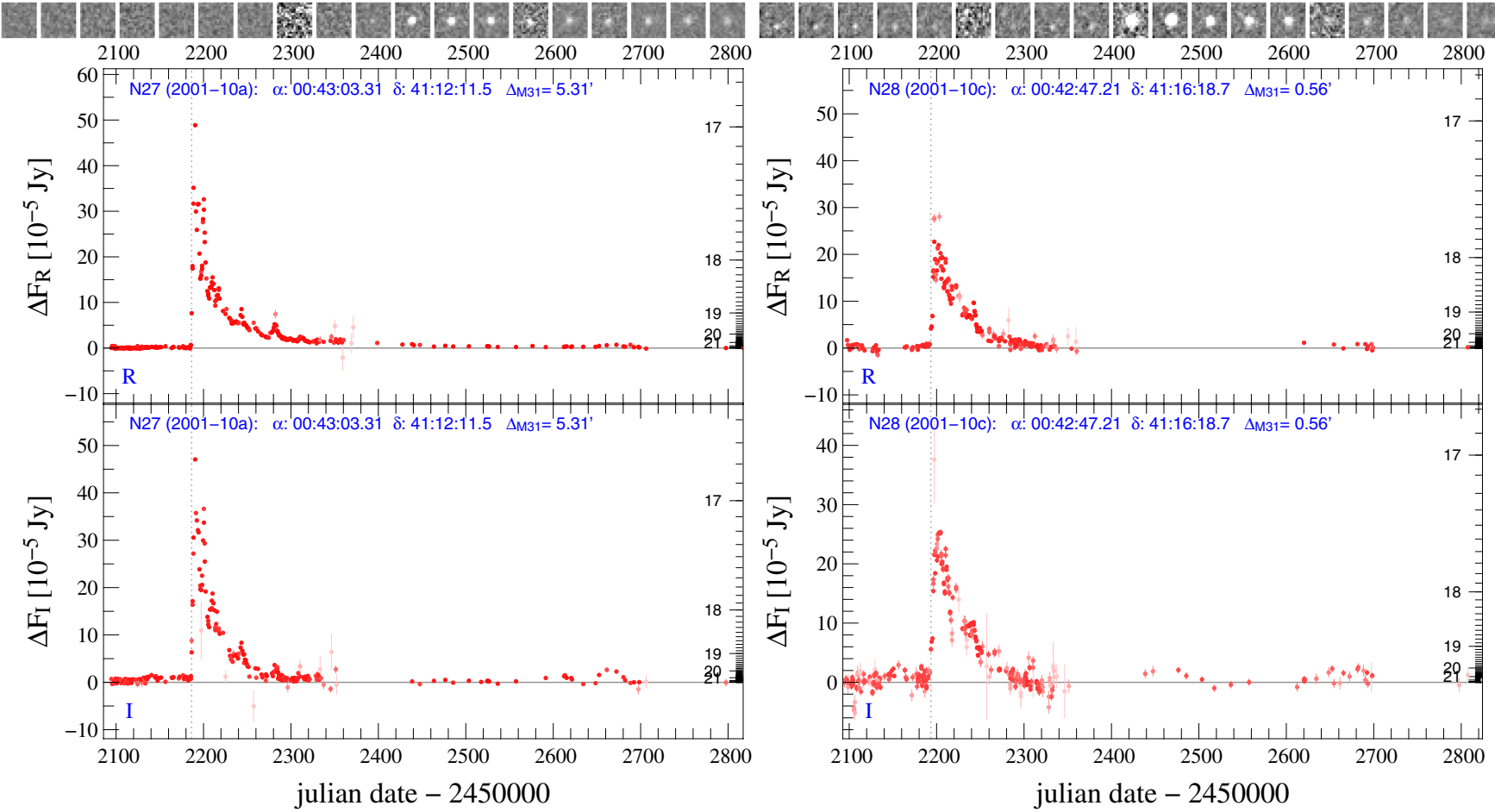

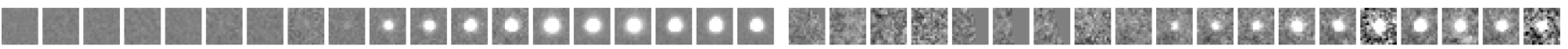

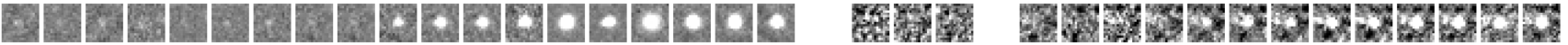

Fig. A.7. Light curve of WeCAPP nova candidates N25, N26, N27 and N28. 
A\&A 537, A43 (2012)
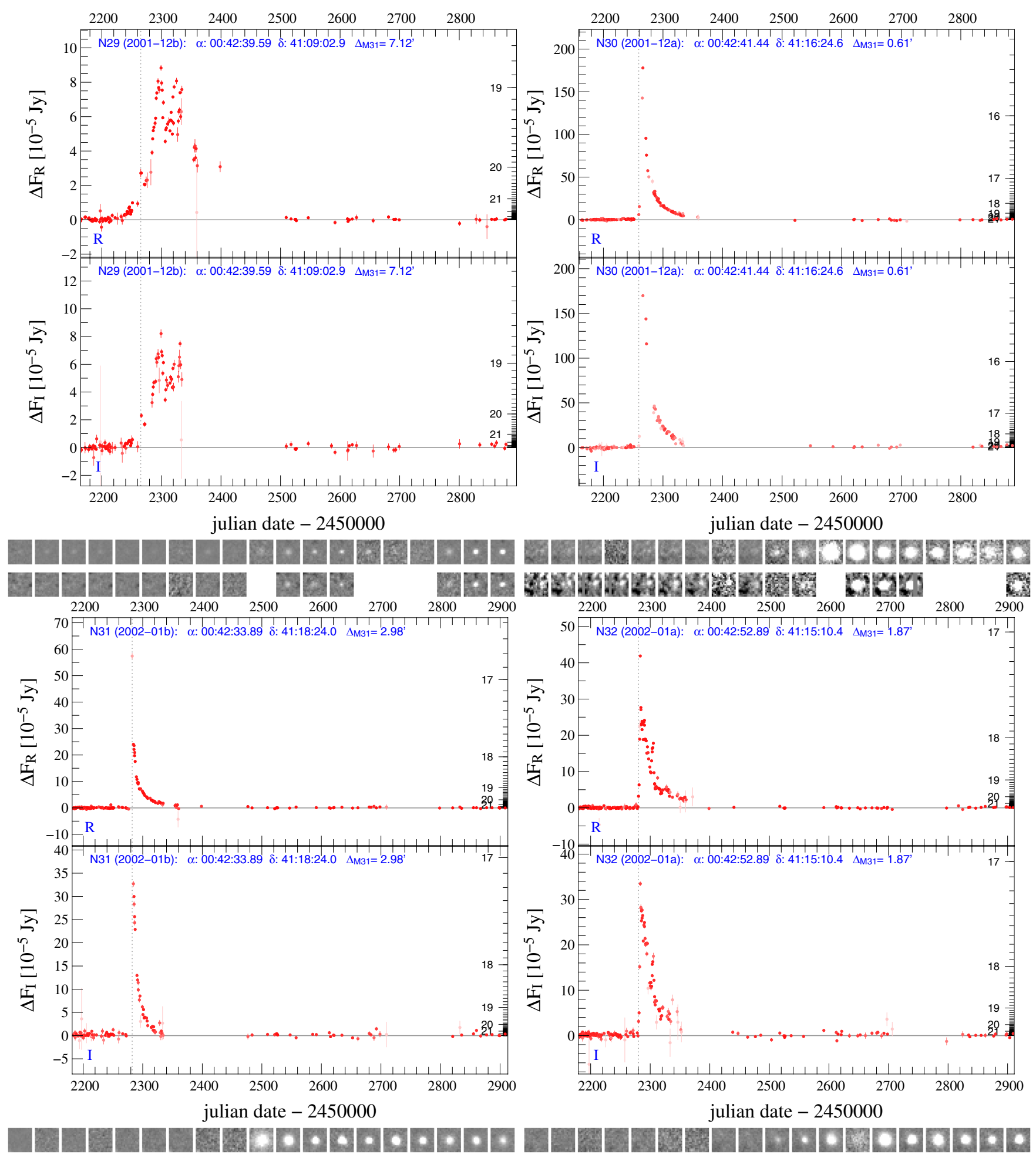

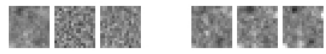

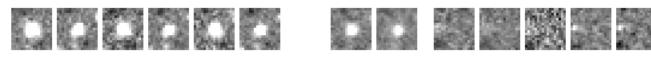

Fig. A.8. Light curve of WeCAPP nova candidates N29, N30, N31 and N32. 
C.-H. Lee et al.: The Wendelstein Calar Alto Pixellensing Project (WeCAPP): the M 31 nova catalogue
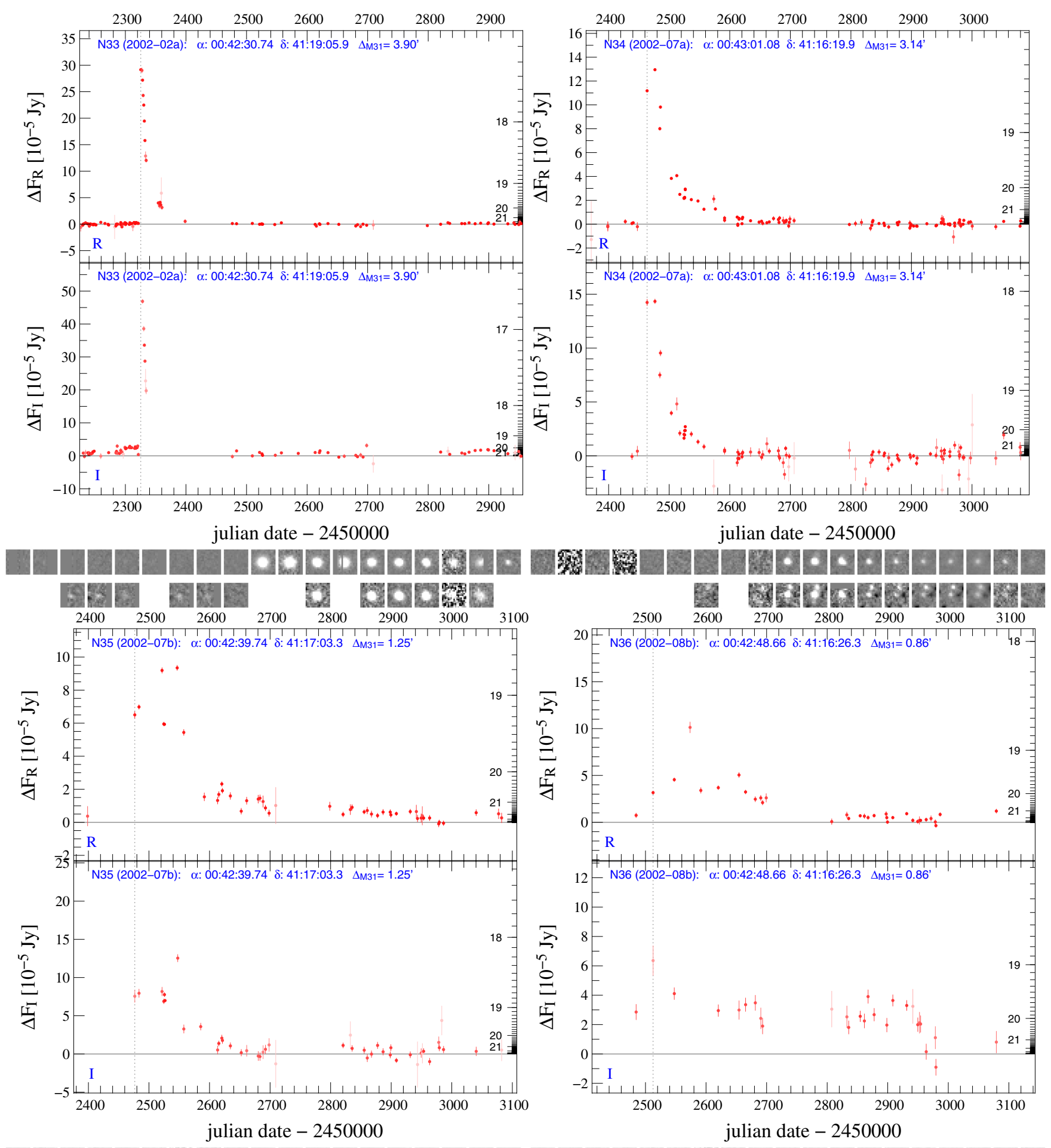

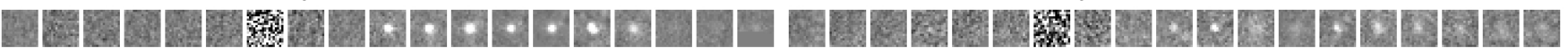

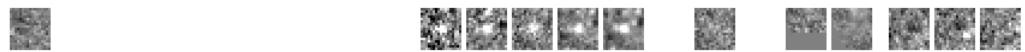

Fig. A.9. Light curve of WeCAPP nova candidates N33, N34, N35 and N36. 
A\&A 537, A43 (2012)
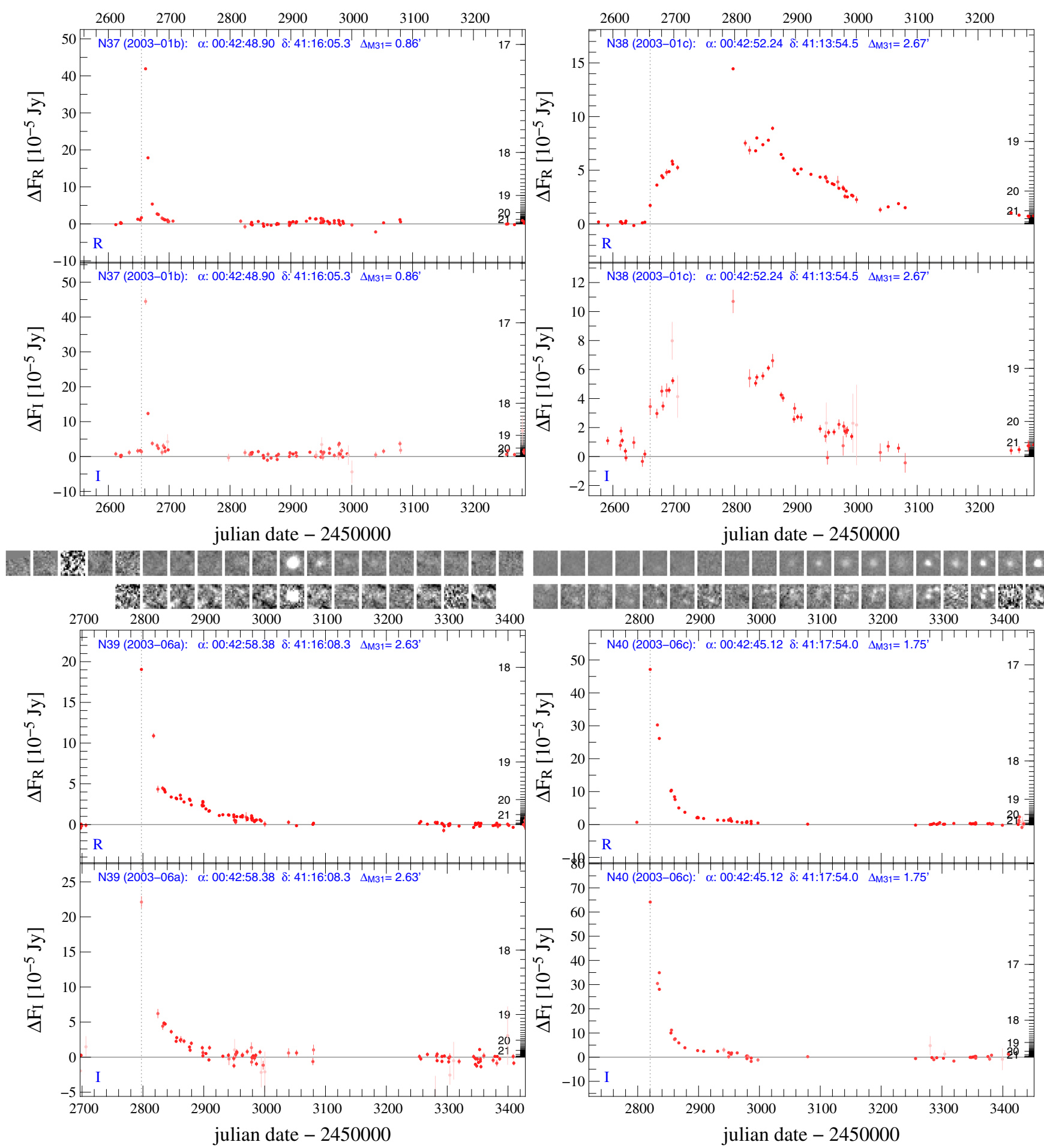

(10 I

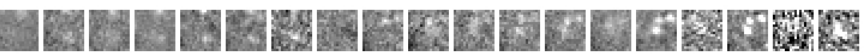

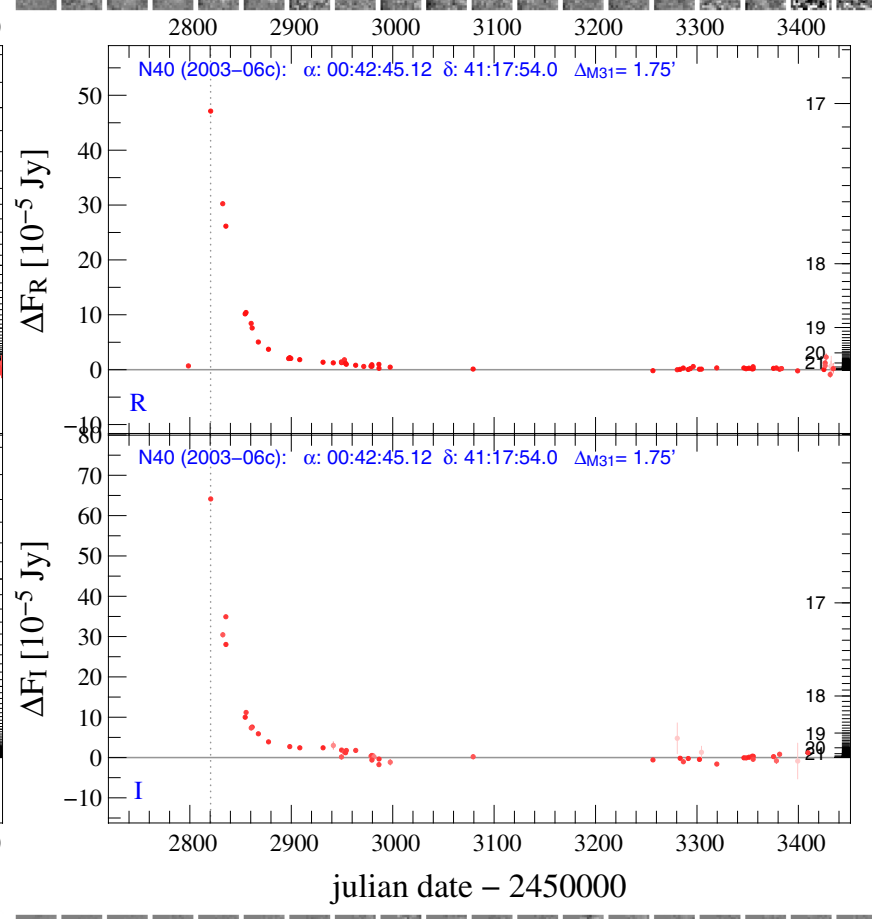

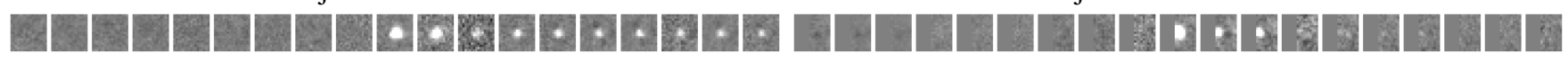

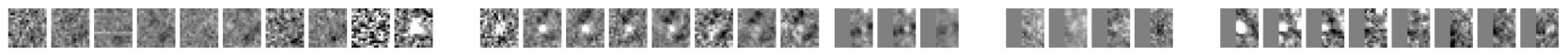

Fig. A.10. Light curve of WeCAPP nova candidates N37, N38, N39 and N40. 
C.-H. Lee et al.: The Wendelstein Calar Alto Pixellensing Project (WeCAPP): the M 31 nova catalogue
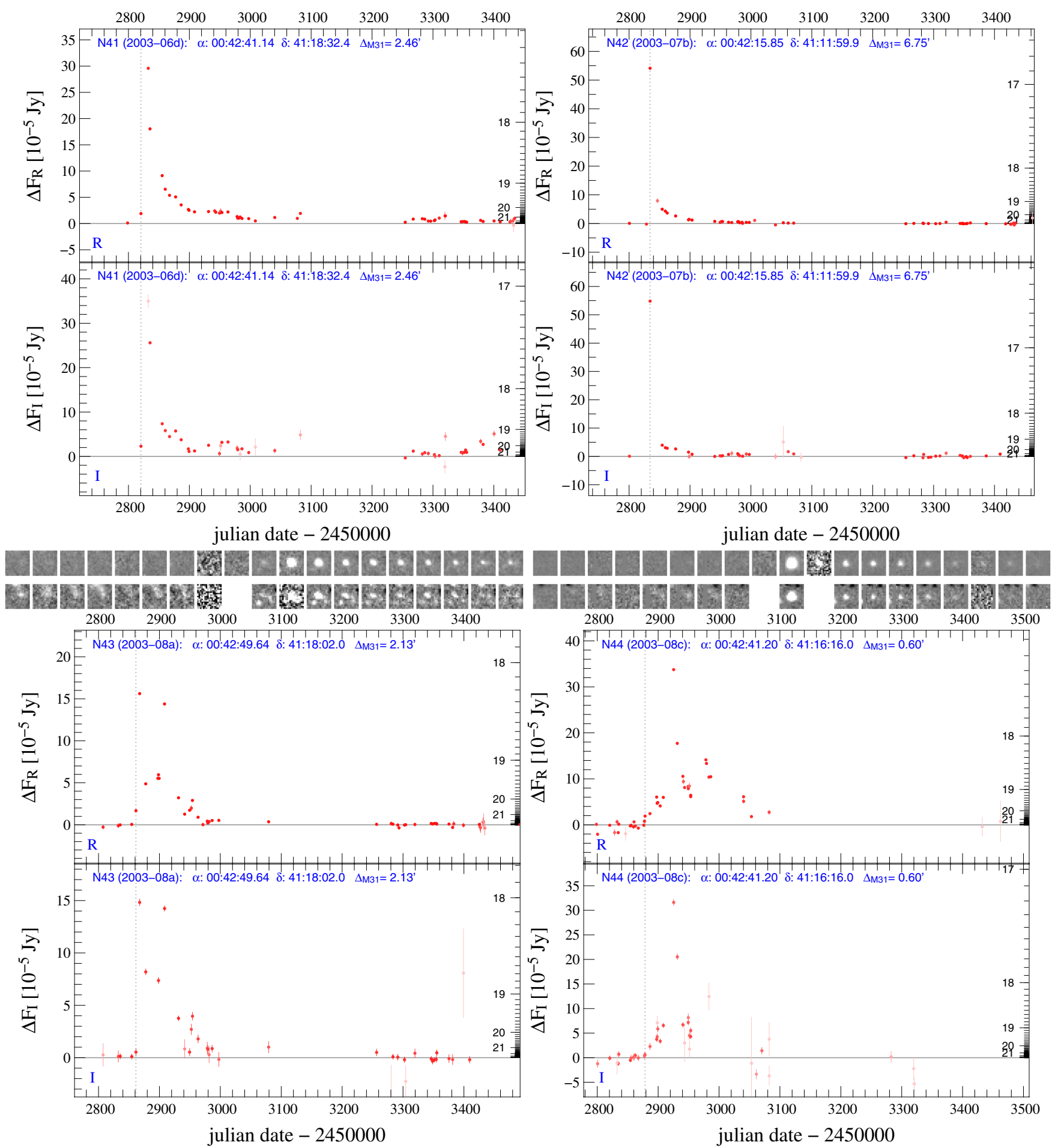

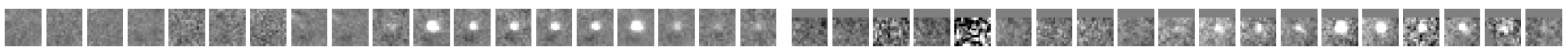

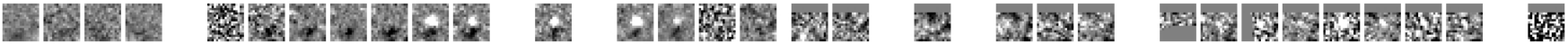

Fig. A.11. Light curve of WeCAPP nova candidates N41, N42, N43 and N44. 
A\&A 537, A43 (2012)
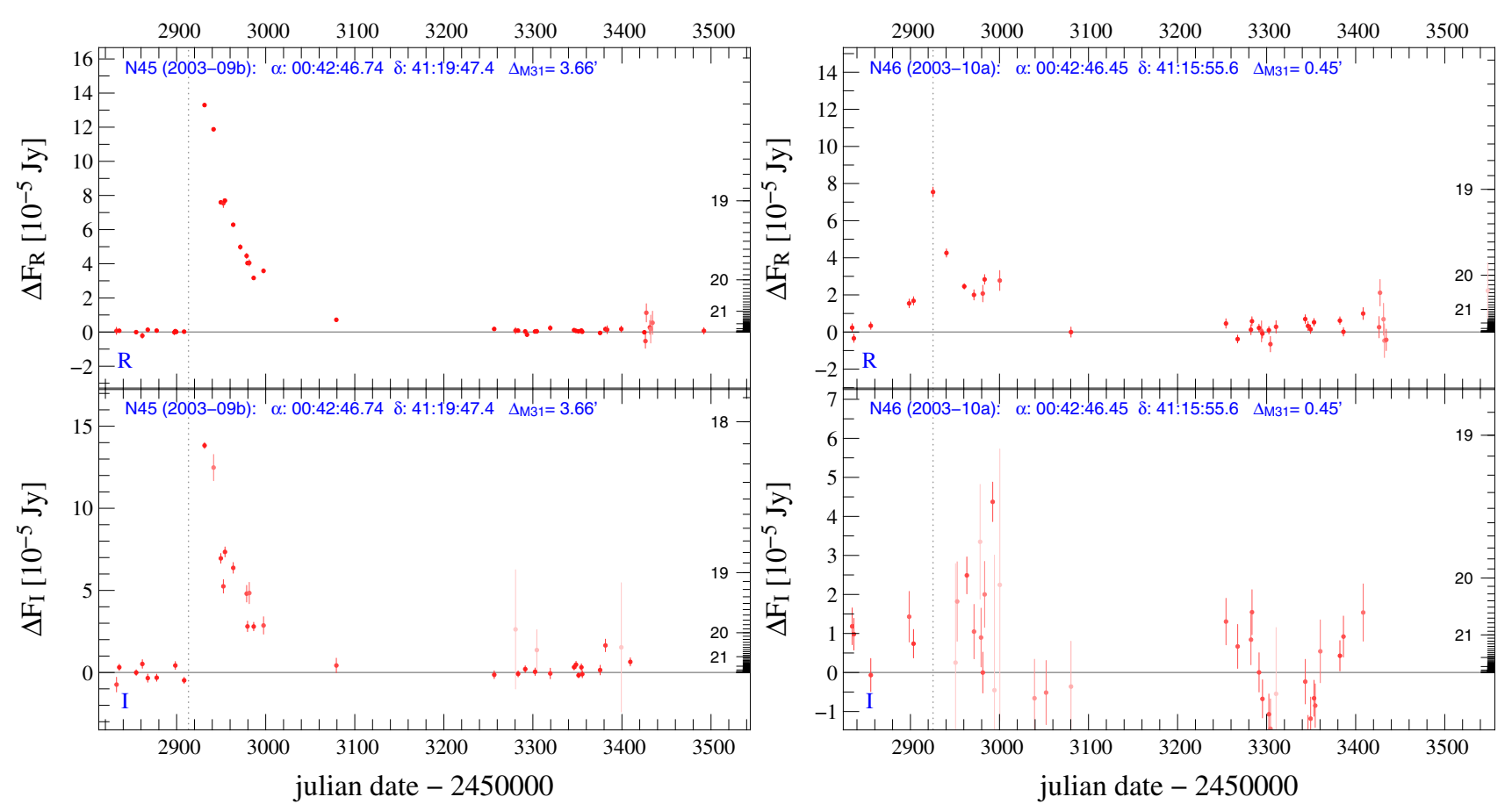

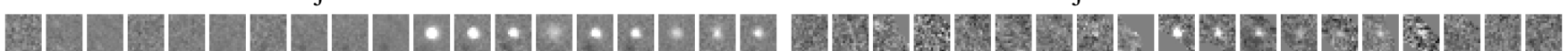

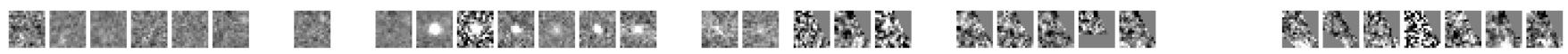
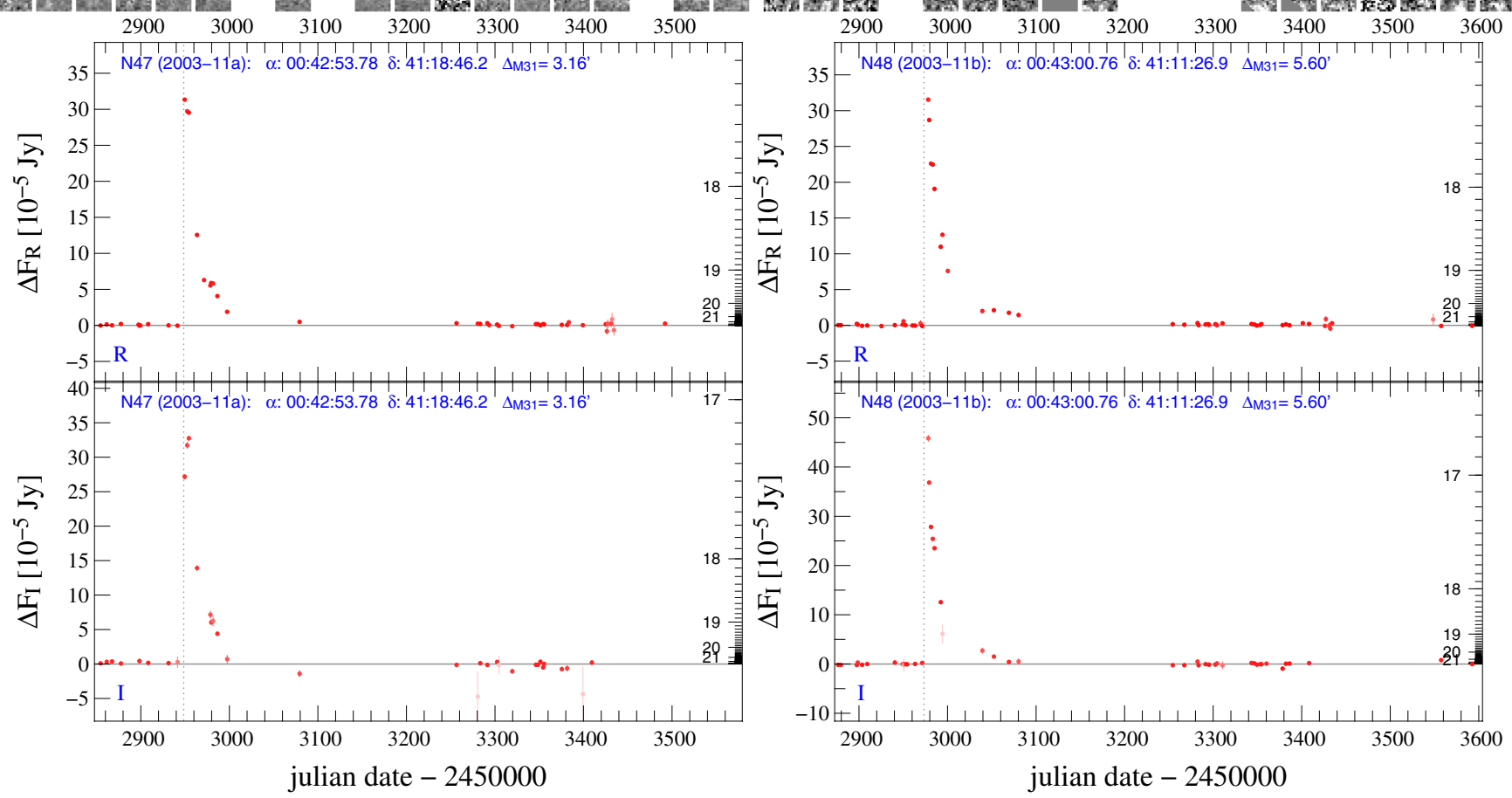

-

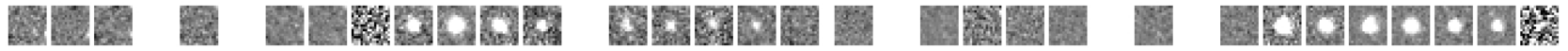

Fig. A.12. Light curve of WeCAPP nova candidates N45, N46, N47 and N48. 
C.-H. Lee et al.: The Wendelstein Calar Alto Pixellensing Project (WeCAPP): the M 31 nova catalogue
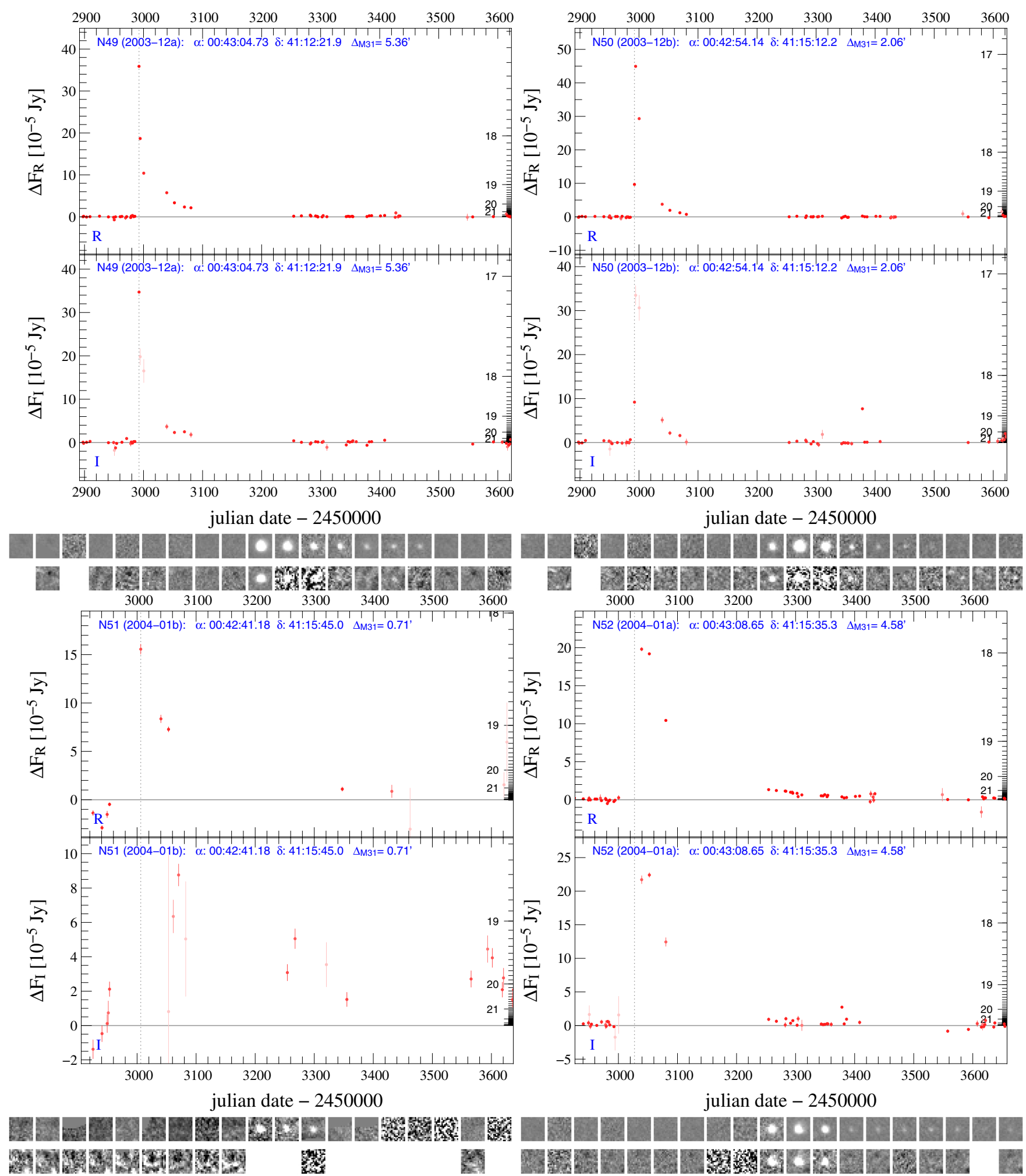

julian date -2450000

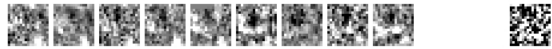

Fig. A.13. Light curve of WeCAPP nova candidates N49, N50, N51 and N52. 

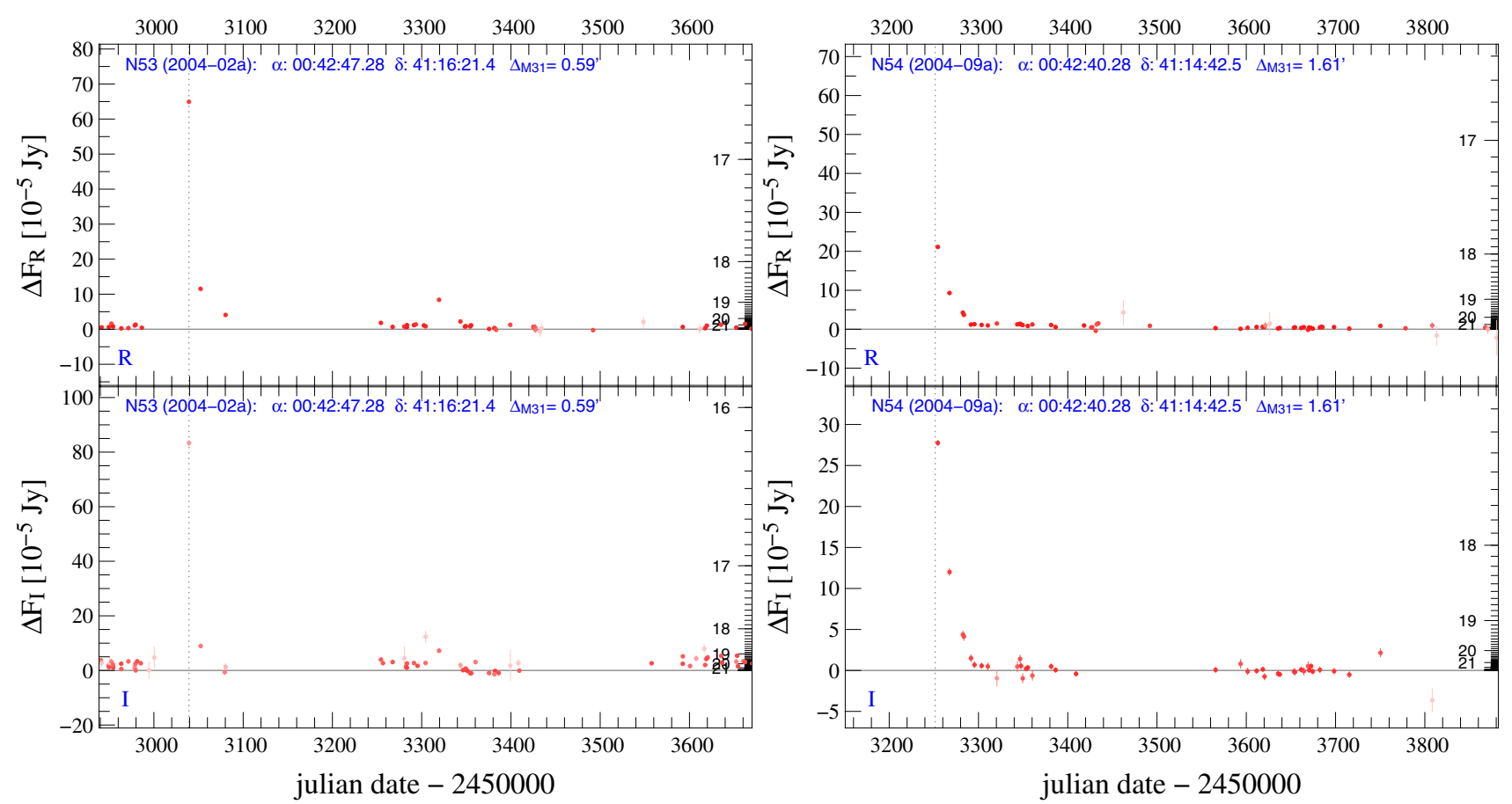

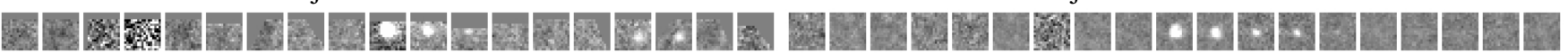
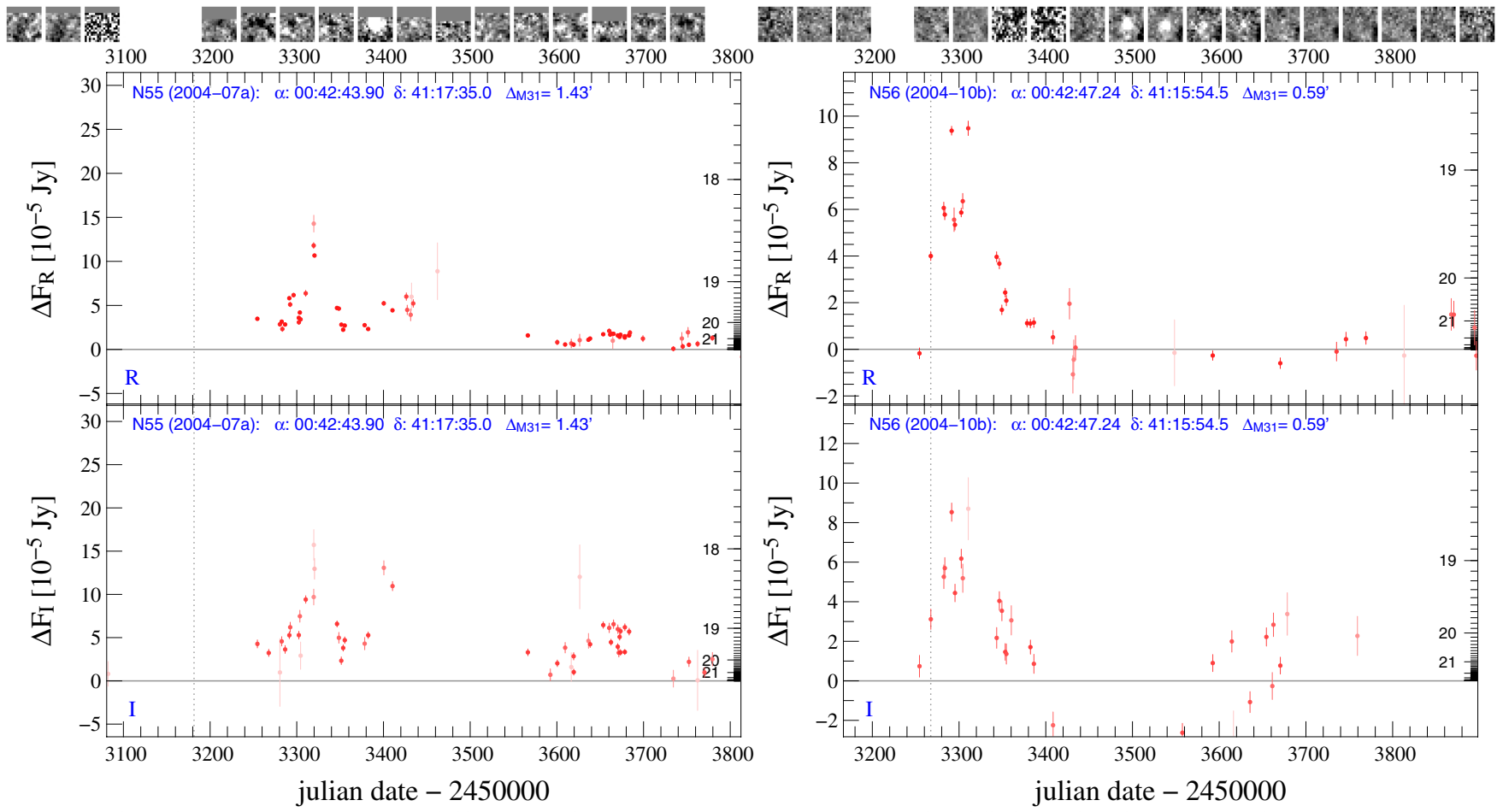

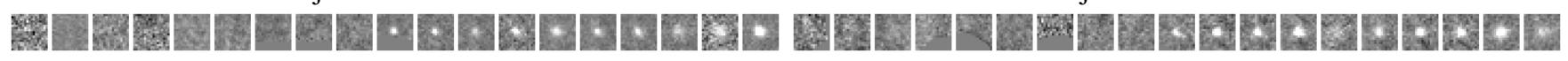

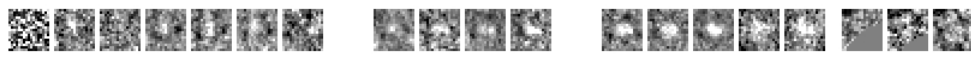

Fig. A.14. Light curve of WeCAPP nova candidates N53, N54, N55 and N56. 
C.-H. Lee et al.: The Wendelstein Calar Alto Pixellensing Project (WeCAPP): the M 31 nova catalogue
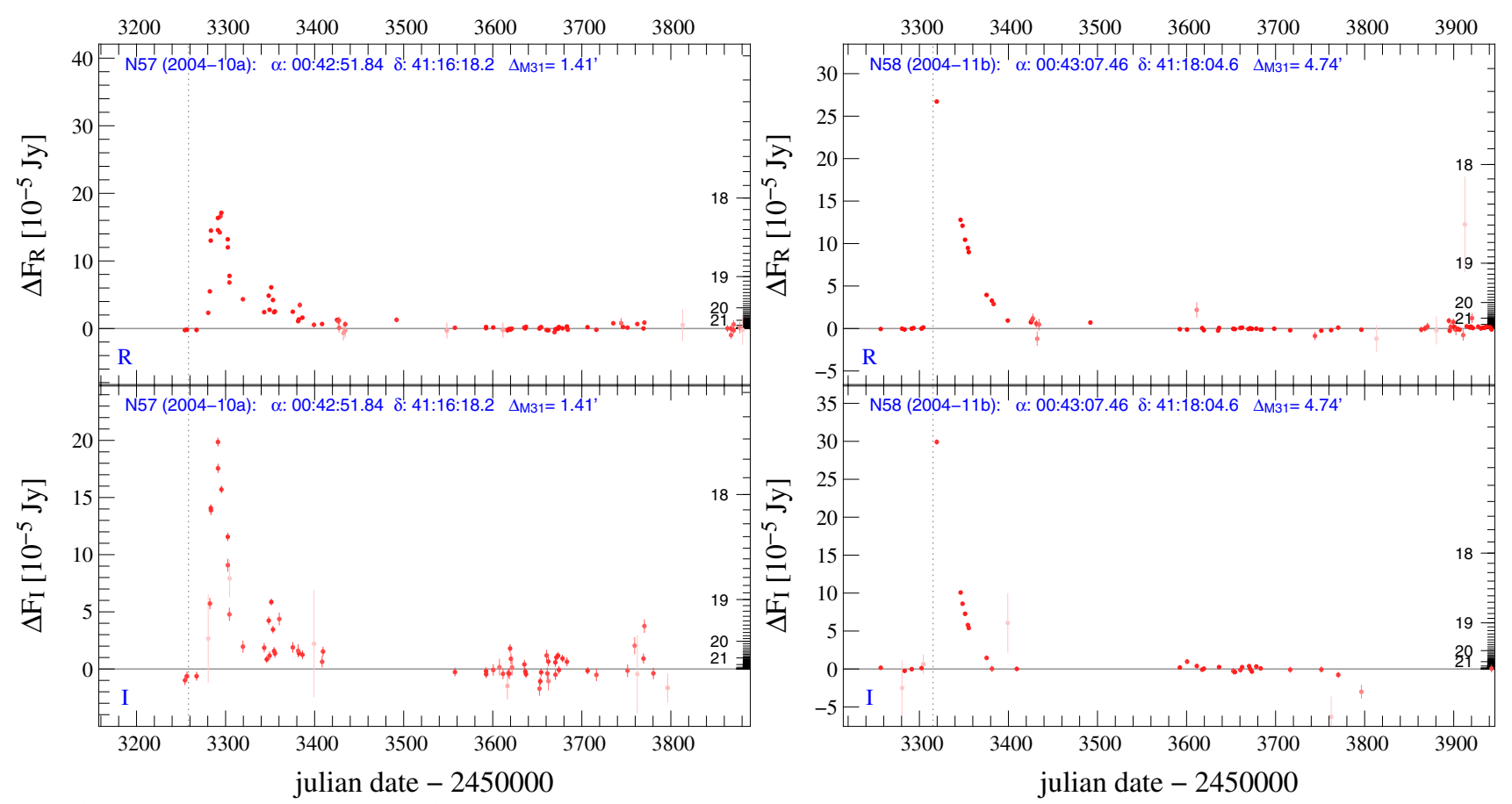

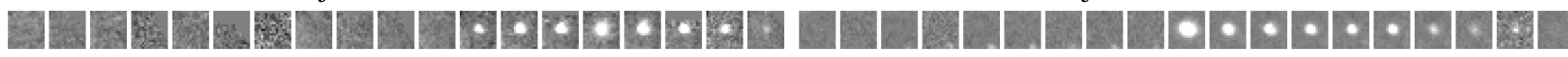
Ga
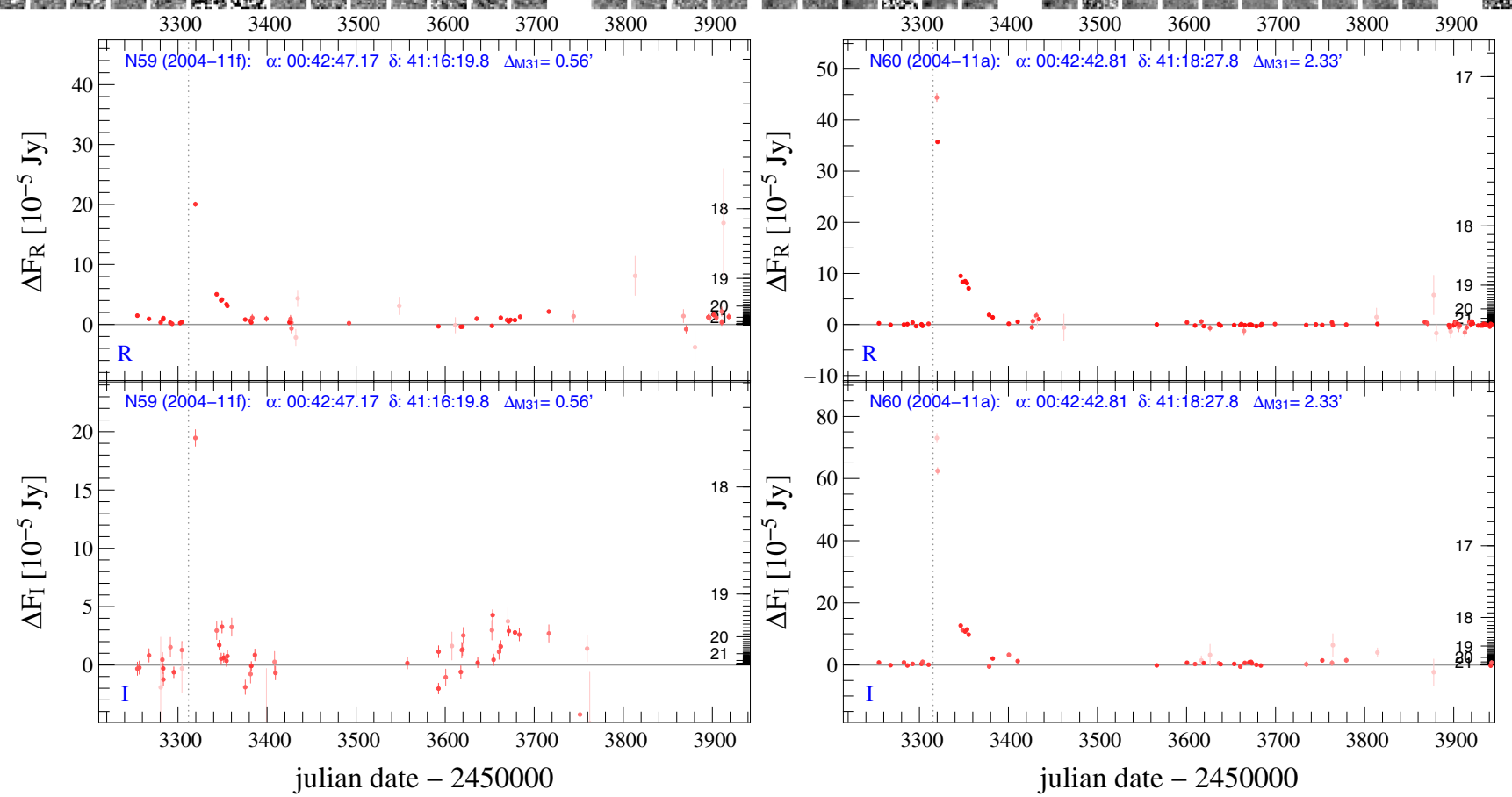

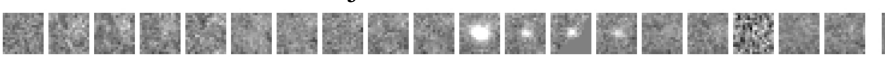

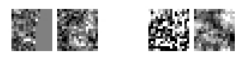
3)

Fig. A.15. Light curve of WeCAPP nova candidates N57, N58, N59 and N60. 
A\&A 537, A43 (2012)
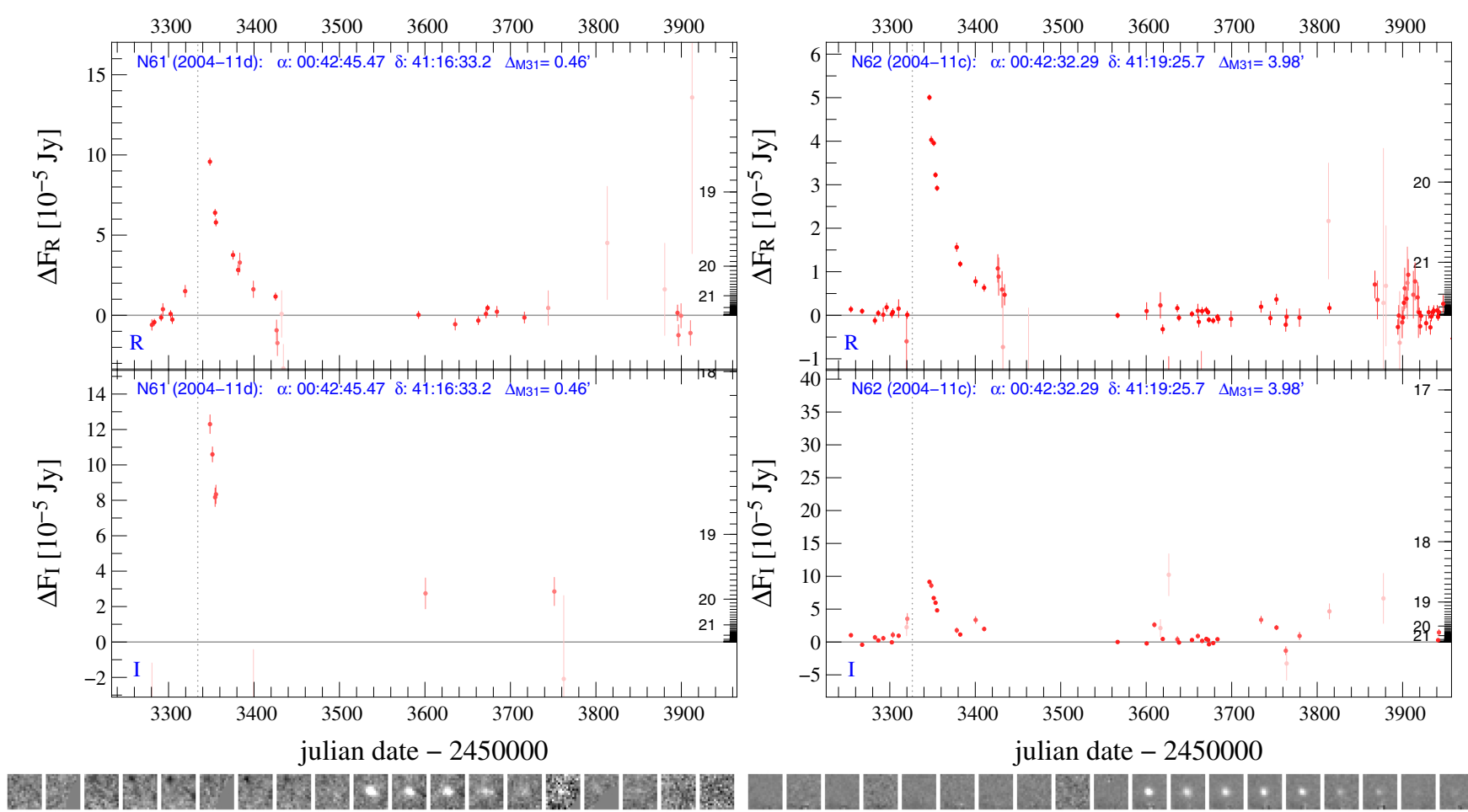

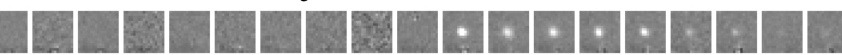
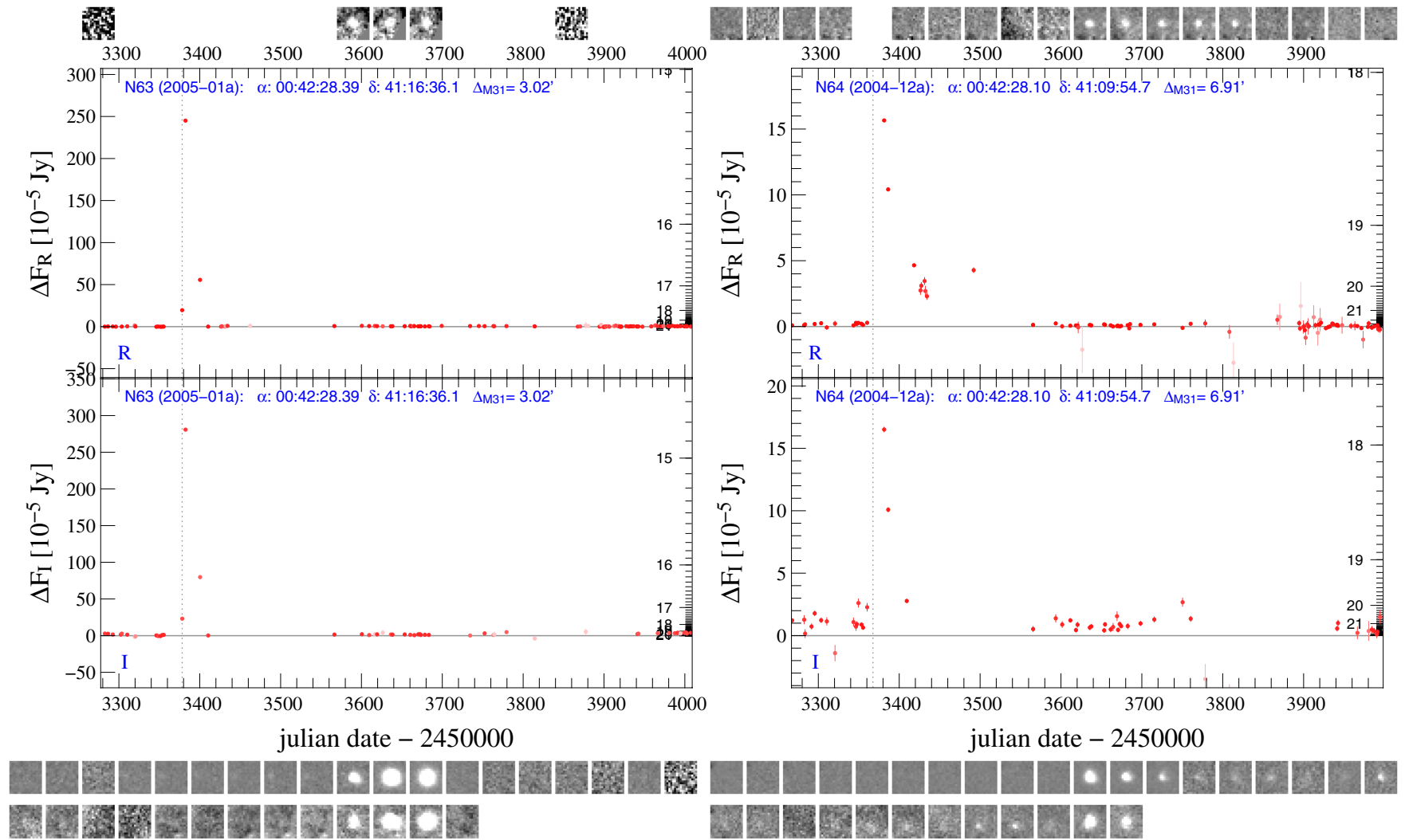

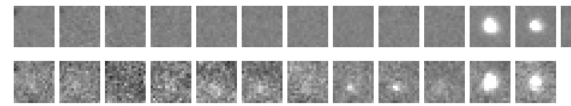

Fig. A.16. Light curve of WeCAPP nova candidates N61, N62, N63 and N64. 
C.-H. Lee et al.: The Wendelstein Calar Alto Pixellensing Project (WeCAPP): the M 31 nova catalogue
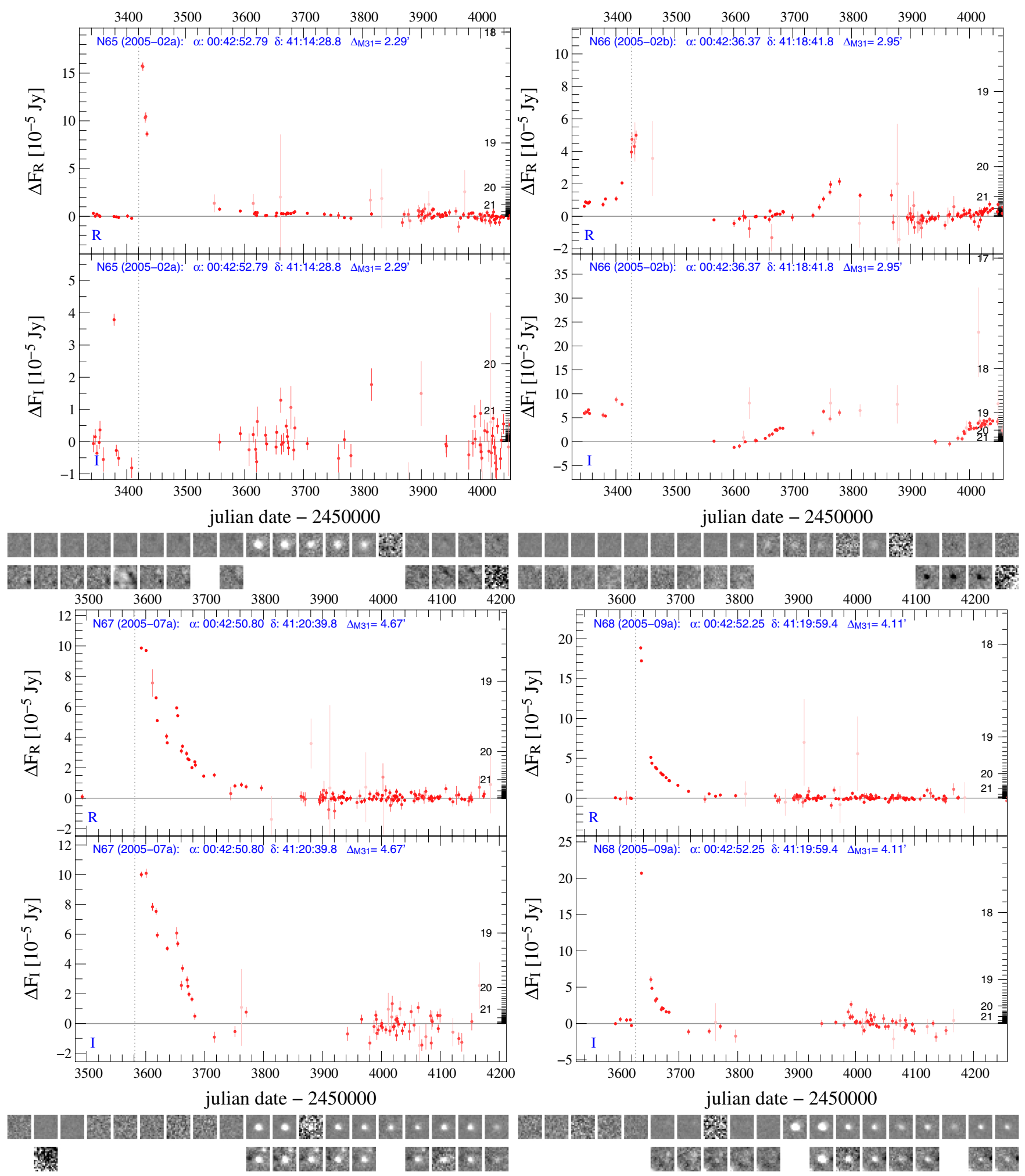

Fig. A.17. Light curve of WeCAPP nova candidates N65, N66, N67 and N68. 
A\&A 537, A43 (2012)
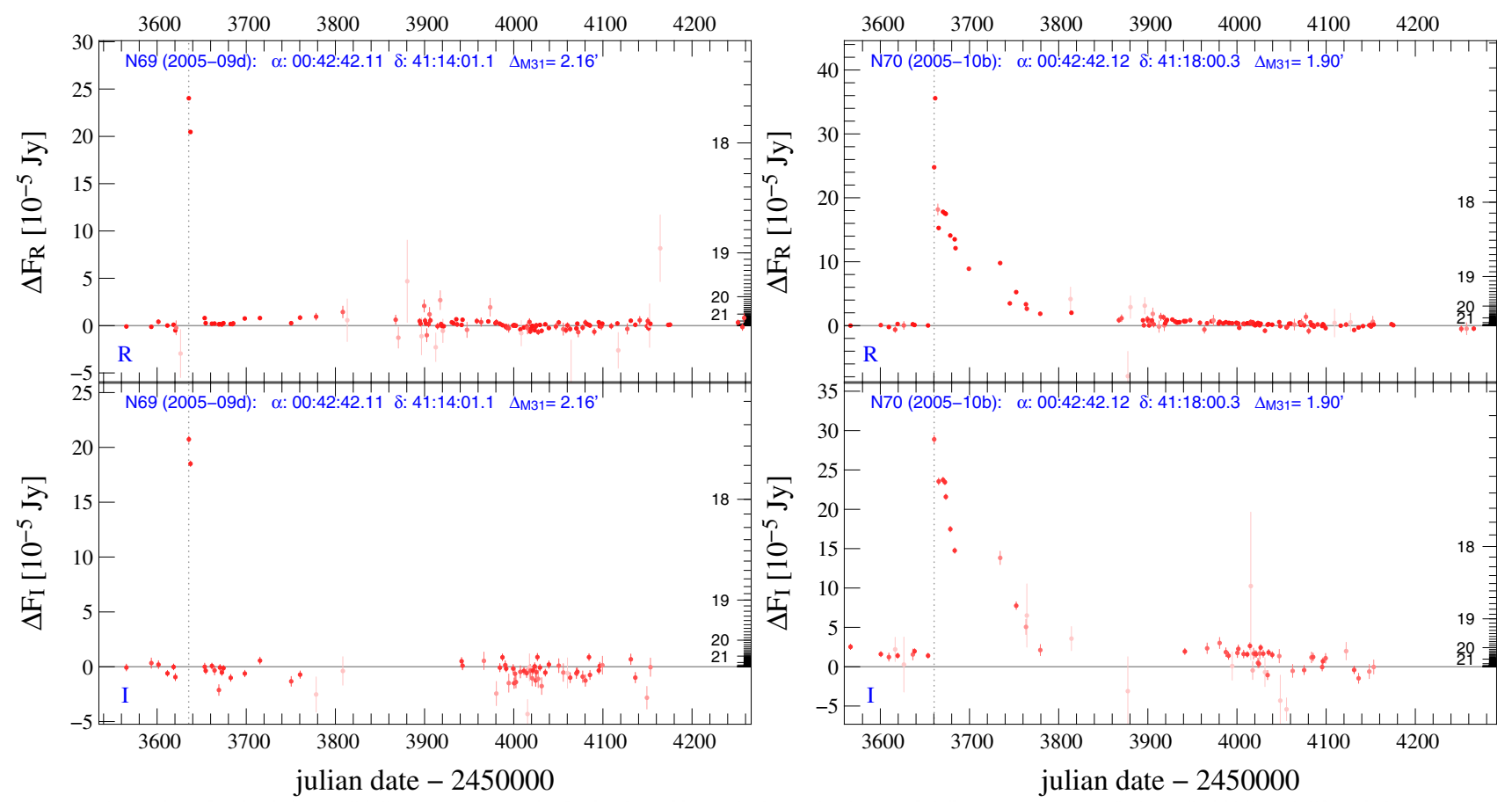

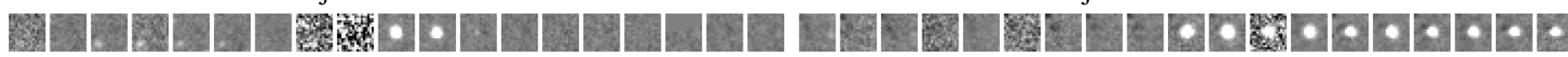

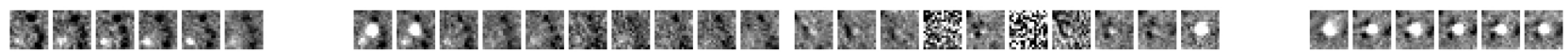
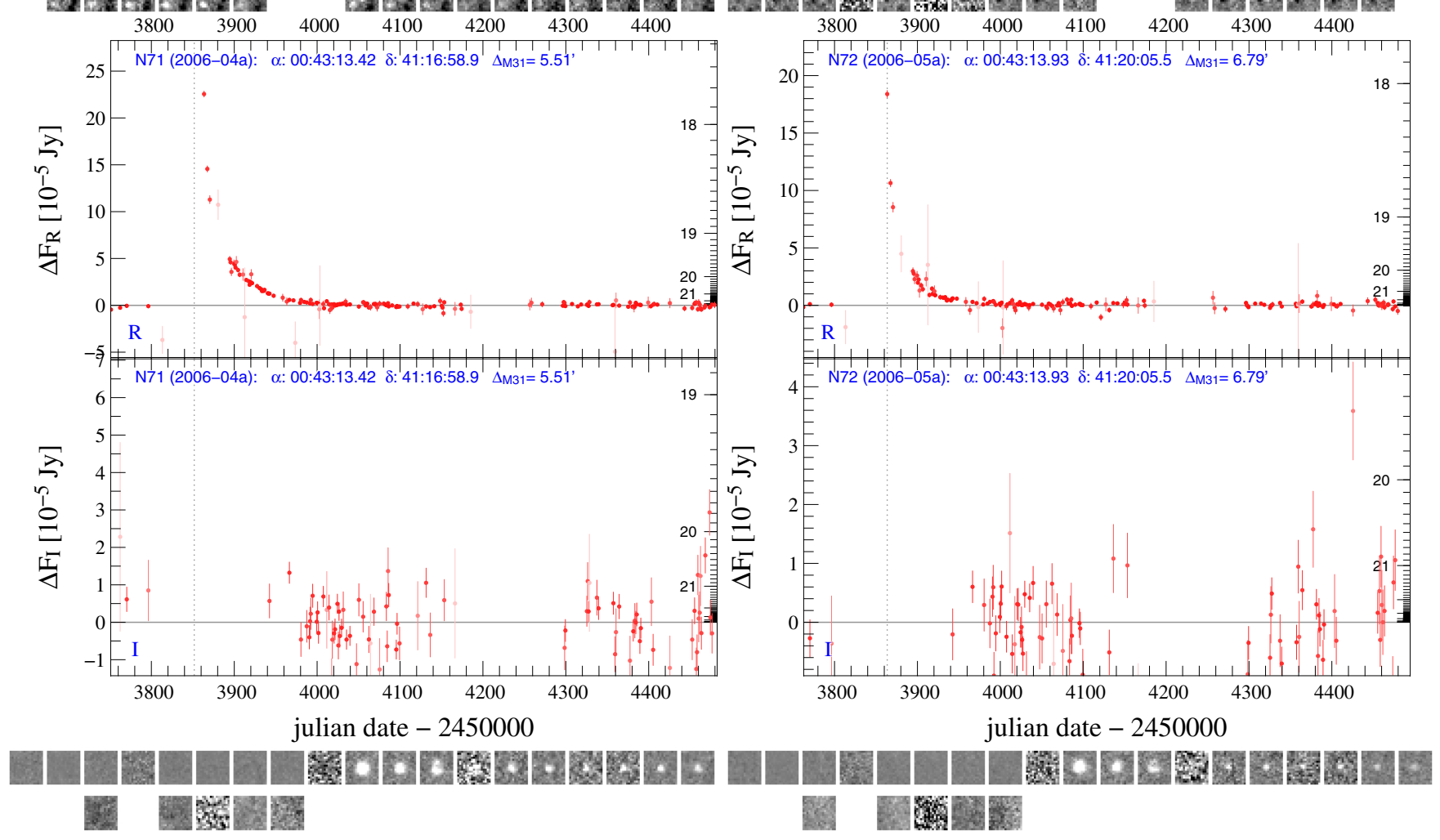

Fig. A.18. Light curve of WeCAPP nova candidates N69, N70, N71 and N72. 
C.-H. Lee et al.: The Wendelstein Calar Alto Pixellensing Project (WeCAPP): the M 31 nova catalogue
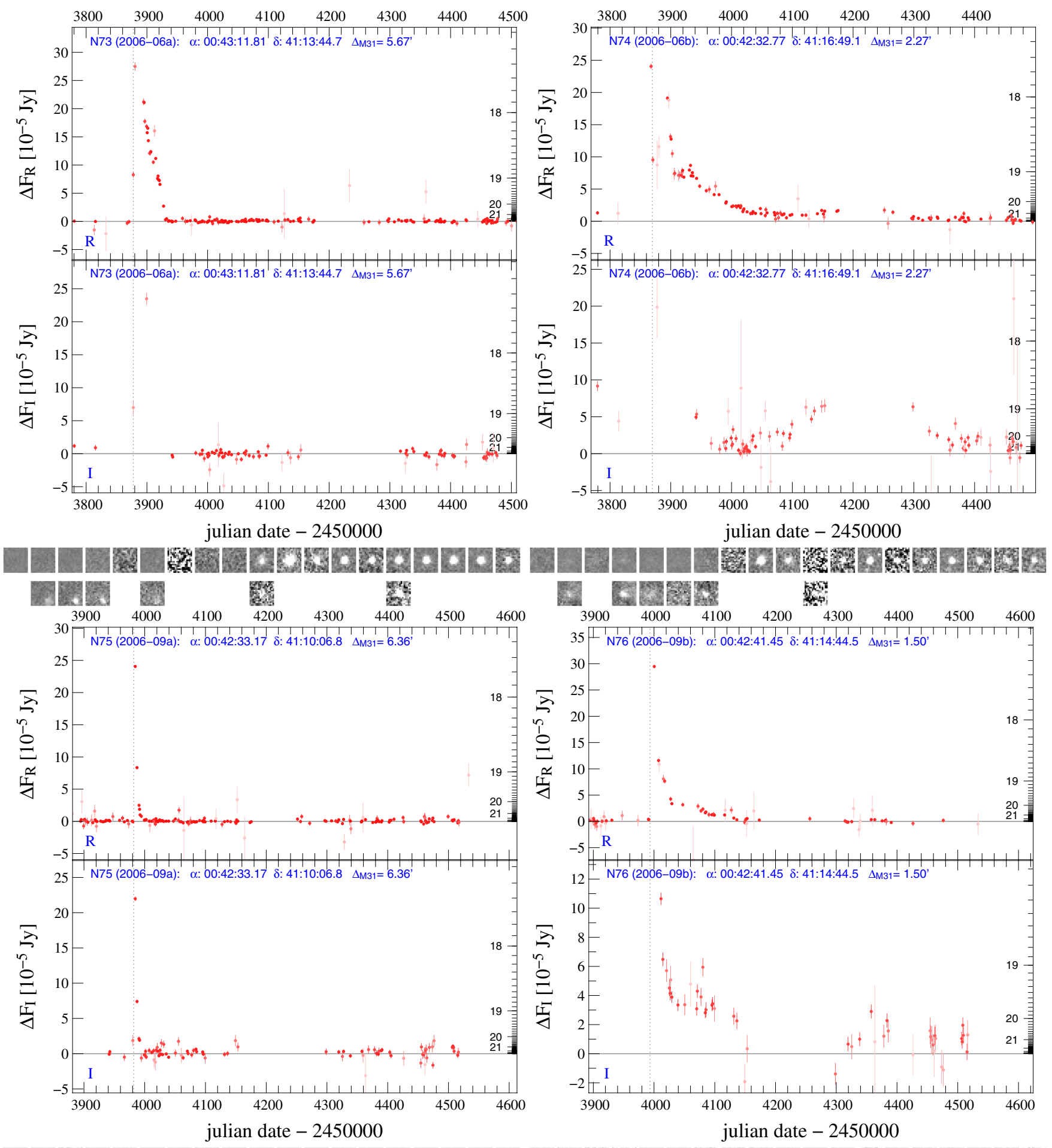

10 D D
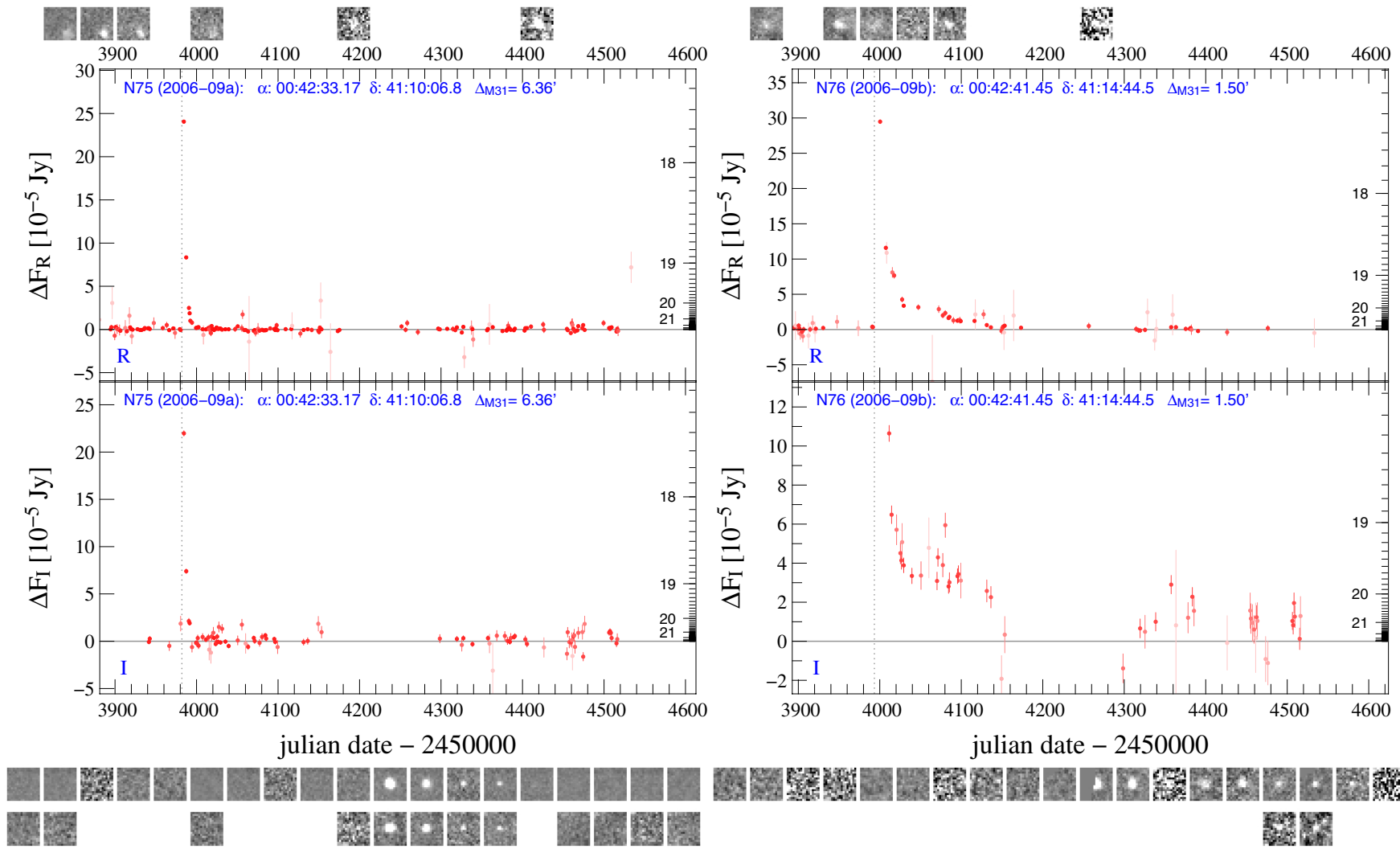

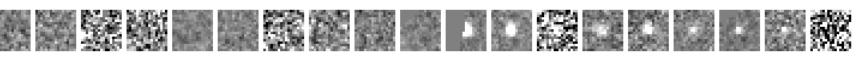

ig. A.19. Light curve of WeCAPP nova candidates N73, N74, N75 and N76. 
A\&A 537, A43 (2012)
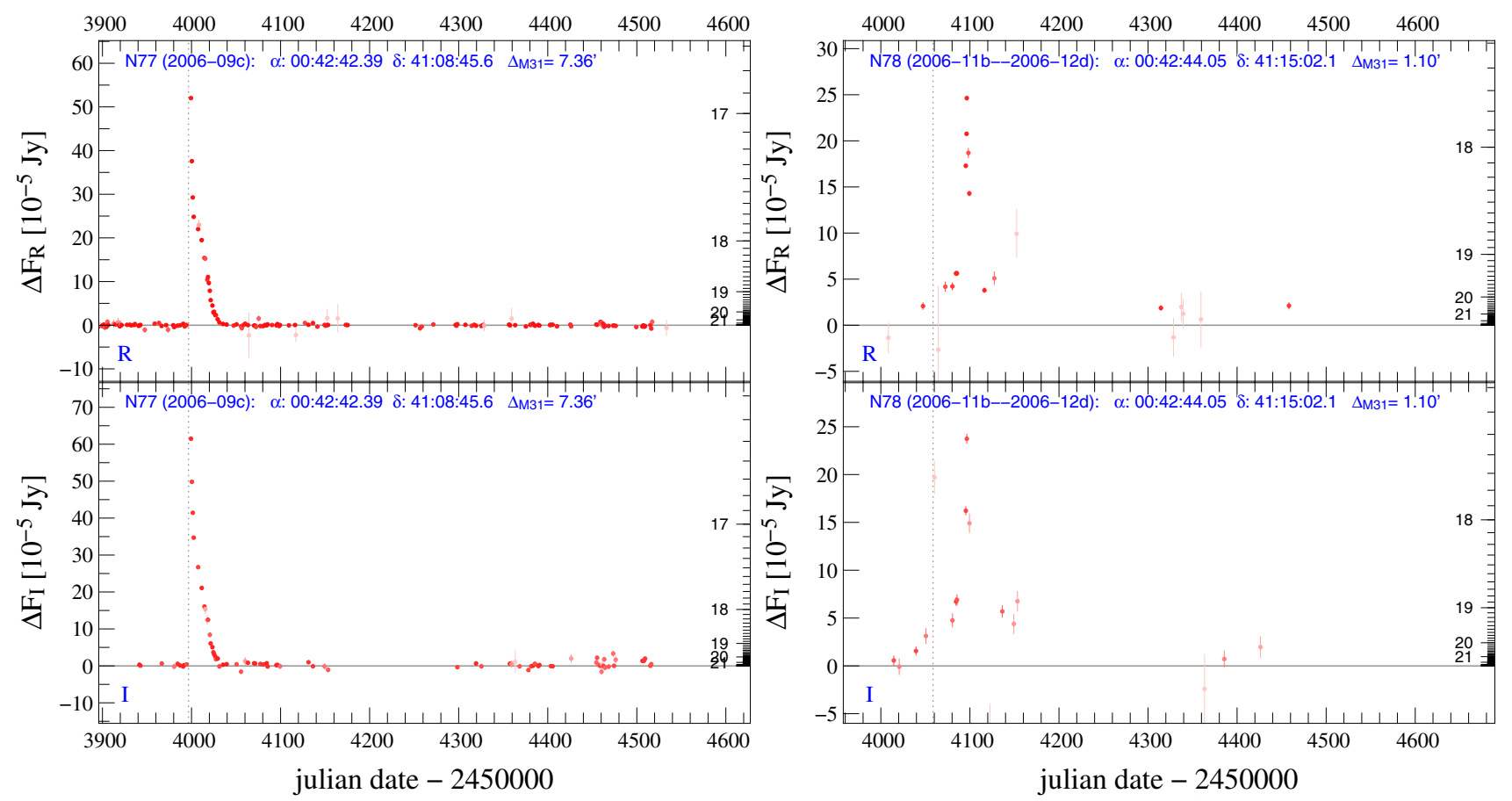

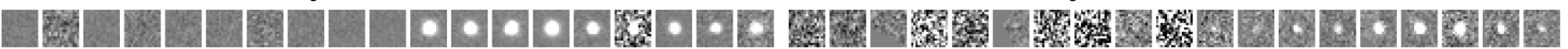
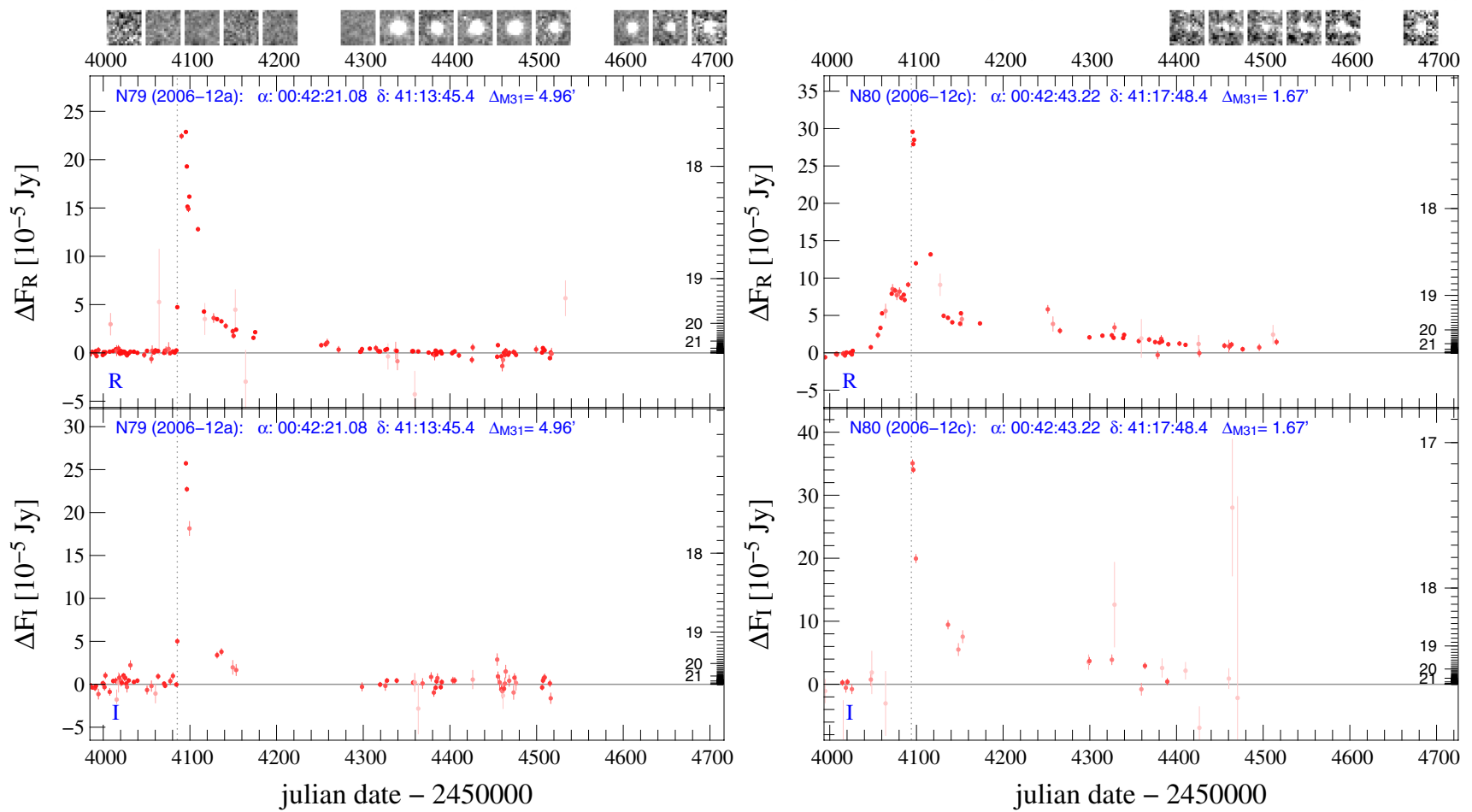

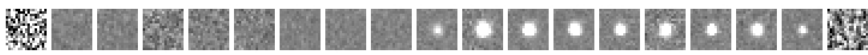

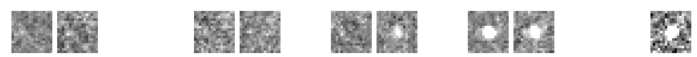

Fig. A.20. Light curve of WeCAPP nova candidates N77, N78, N79 and N80. 
C.-H. Lee et al.: The Wendelstein Calar Alto Pixellensing Project (WeCAPP): the M 31 nova catalogue
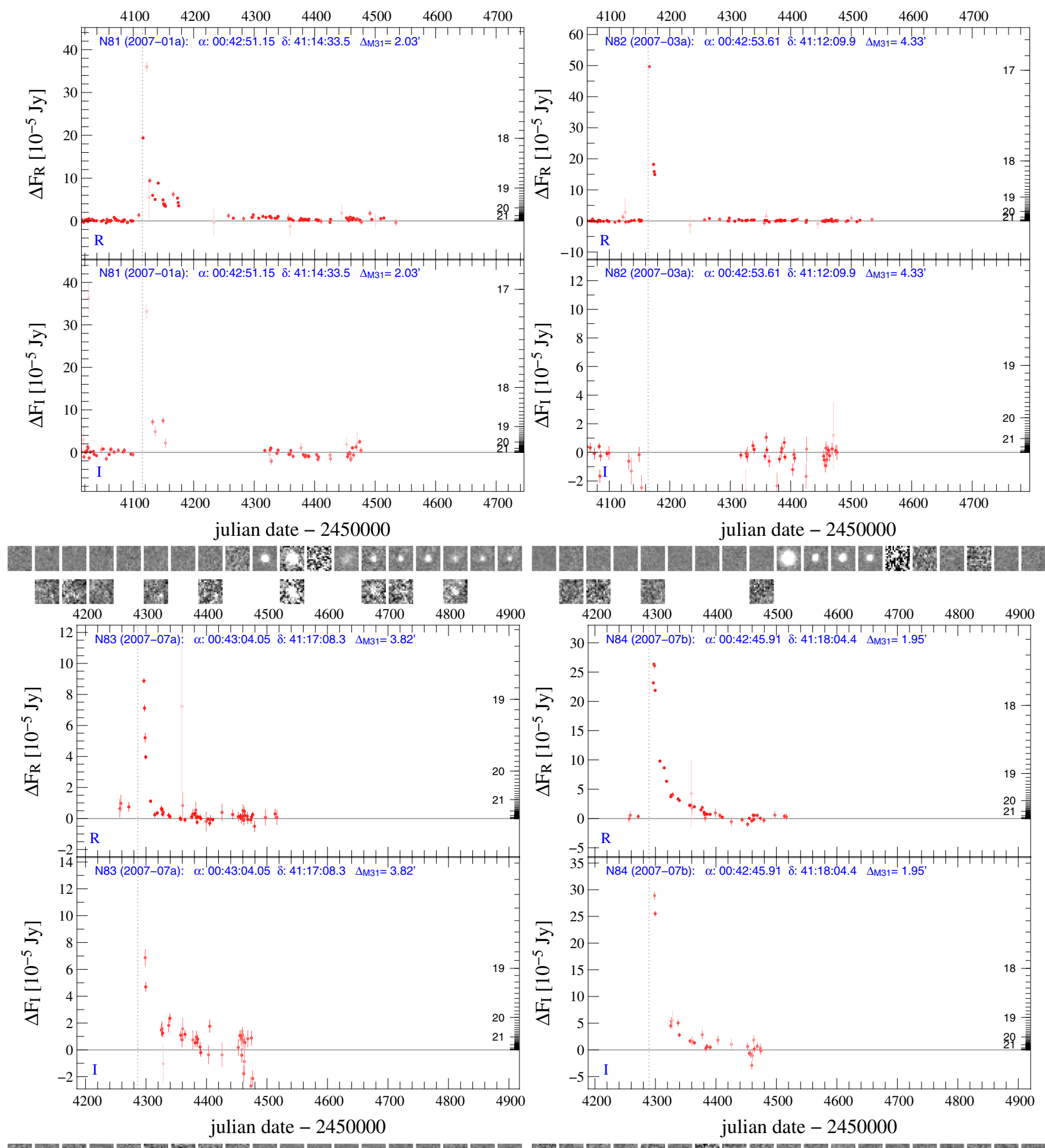

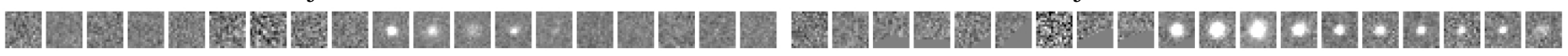

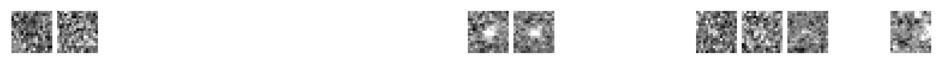

Fig. A.21. Light curve of WeCAPP nova candidates N81, N82, N83 and N84. 
A\&A 537, A43 (2012)
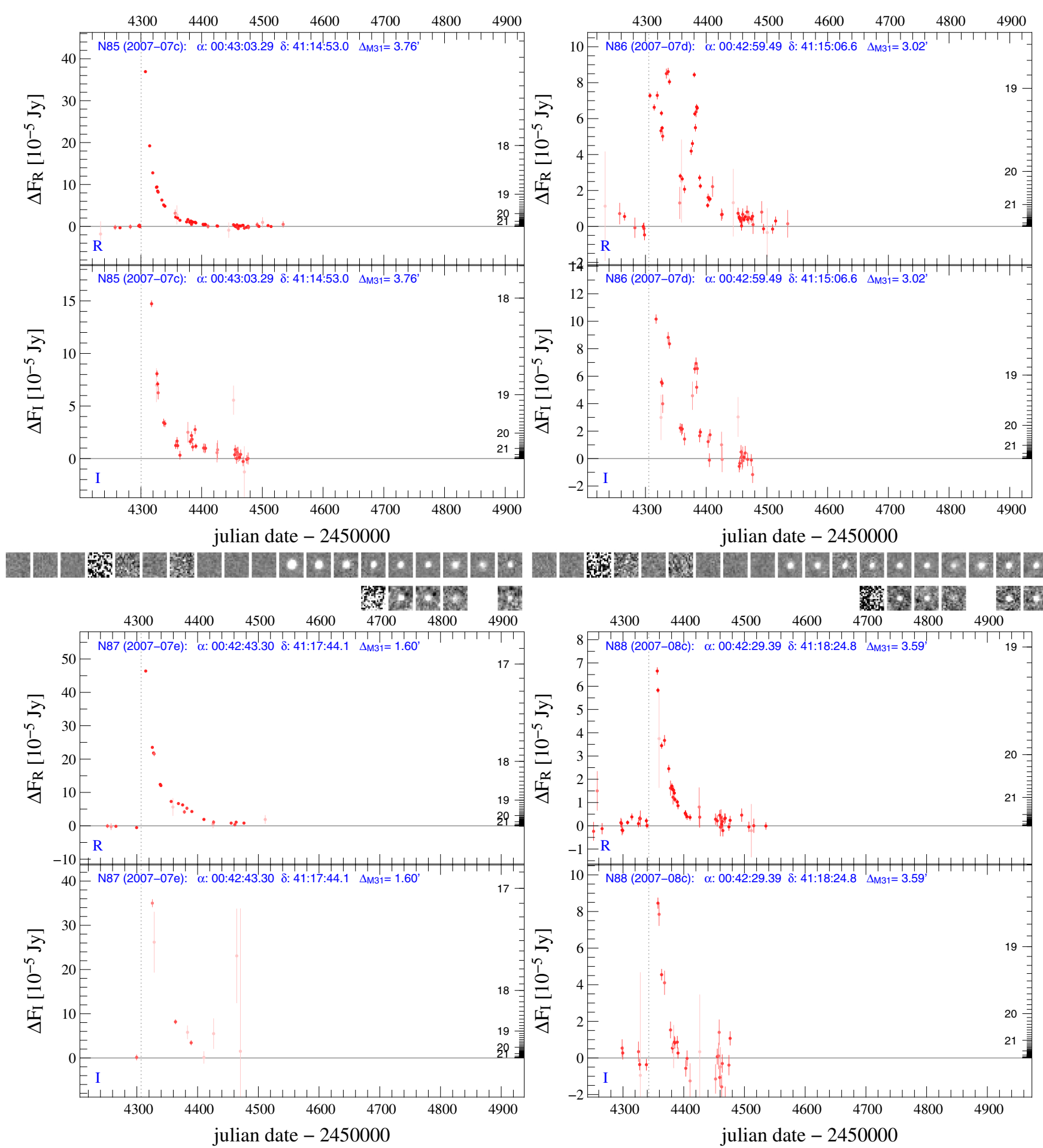

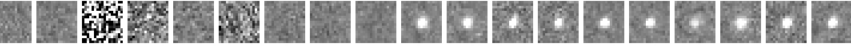

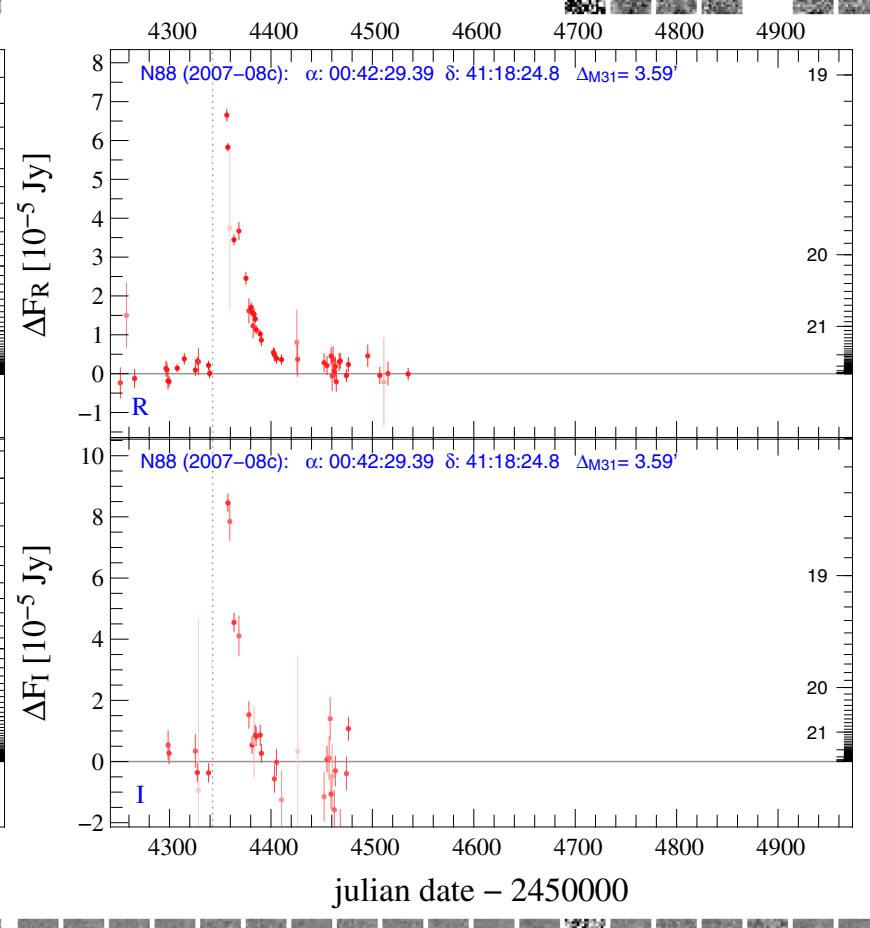

A a 12 程

Fig. A.22. Light curve of WeCAPP nova candidates N85, N86, N87 and N88. 
C.-H. Lee et al.: The Wendelstein Calar Alto Pixellensing Project (WeCAPP): the M 31 nova catalogue

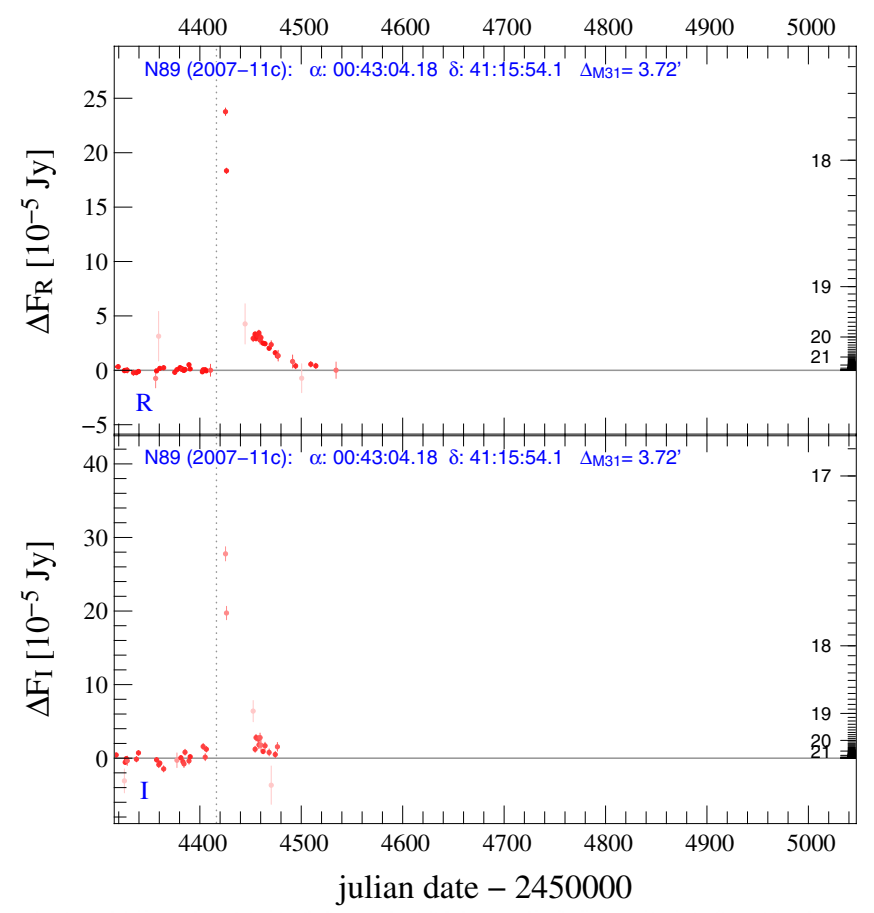

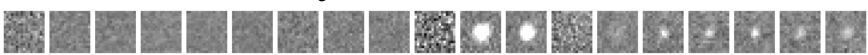
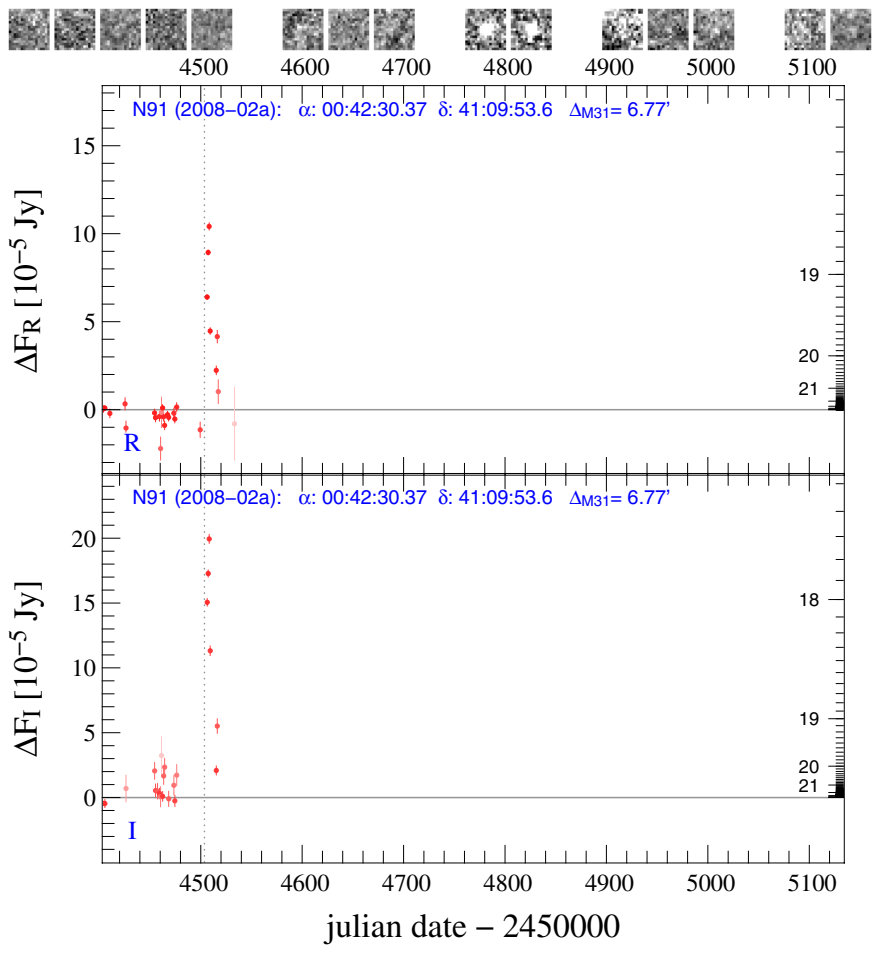

\section{4.}

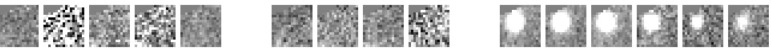

Fig. A.23. Light curve of WeCAPP nova candidates N89, N90, and N91.

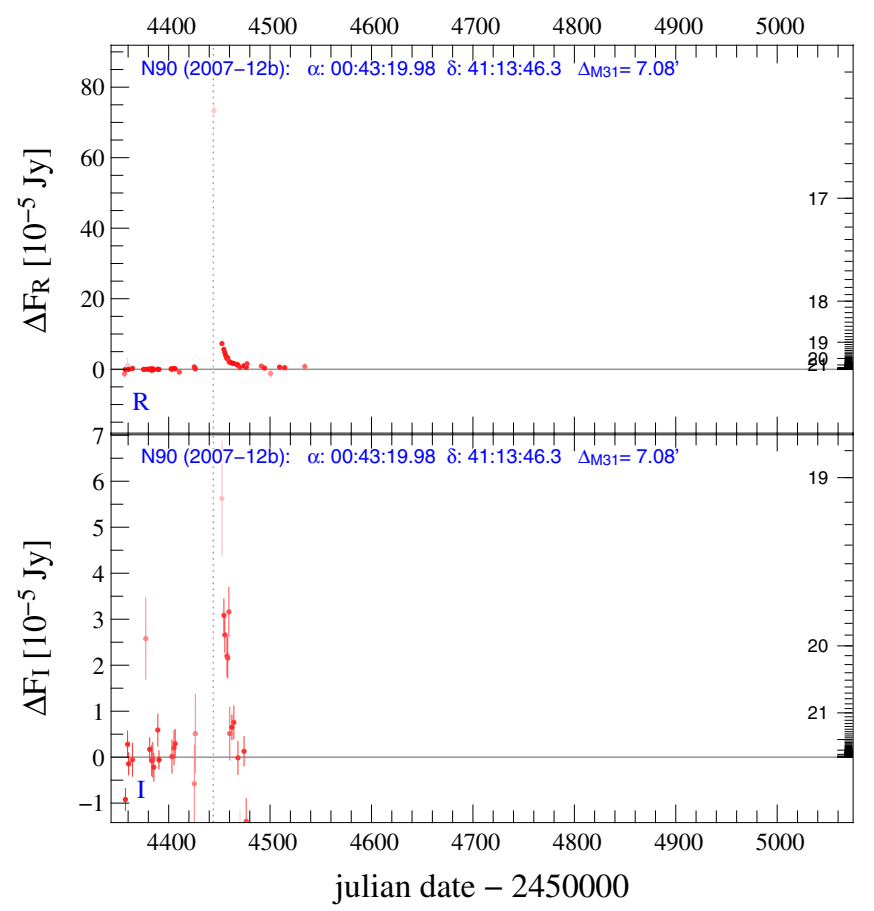

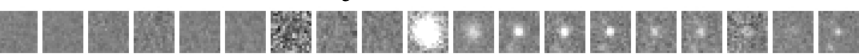

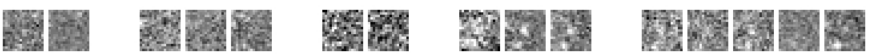




\section{Appendix B: Light curves of nova candidates from literature}

Here we present light curves for nova candidates that are reported by other group(s) in M 31 within the field of view and time span of WeCAPP project but which do not pass our nova detection criteria. The name, position, and distance to the centre of M 31 are shown in the figures. The time of outburst according to Pietsch et al. (2007) and Pietsch (2010) is indicated by the vertical grey line. For each nova candidate we investigated why it did not fulfil our nova detection criteria. We group the nova candidates according to our findings and summarize these findings for each of these groups in the following. For 3 nova candidates (2003-08b, 2004-09b, 2007-06a) we have no data points in the WeCAPP dataset.

B.1. Nova candidates that are not detected by WeCAPP 
C.-H. Lee et al.: The Wendelstein Calar Alto Pixellensing Project (WeCAPP): the M 31 nova catalogue
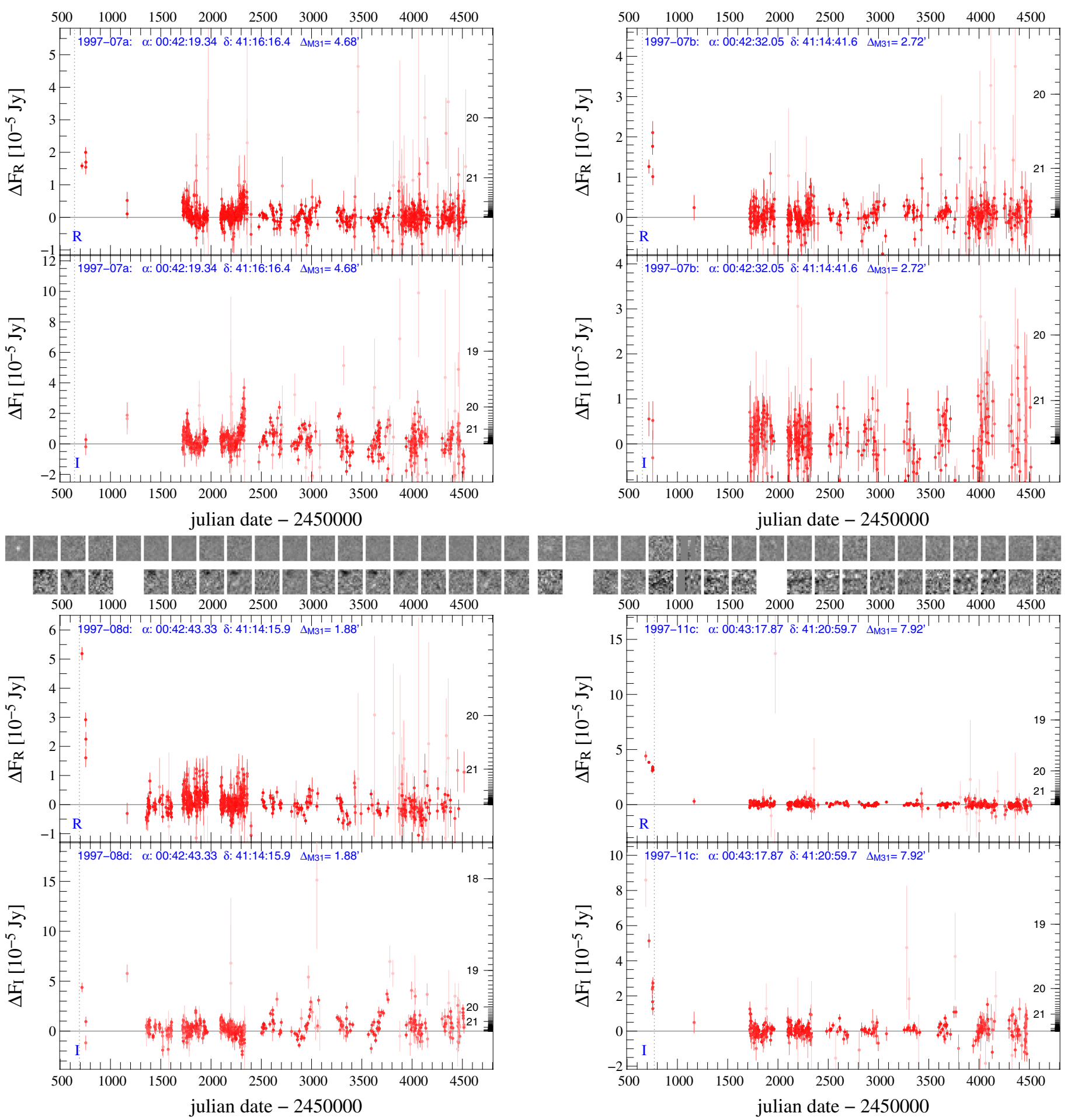

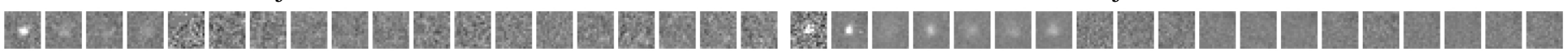

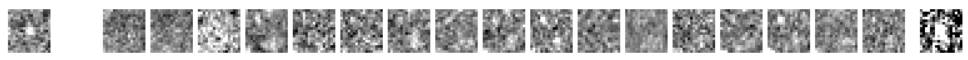

Fig. B.1. Light curve of 1997-07a, 1997-07b, 1997-08d, and 1997-11c from WeCAPP data. 

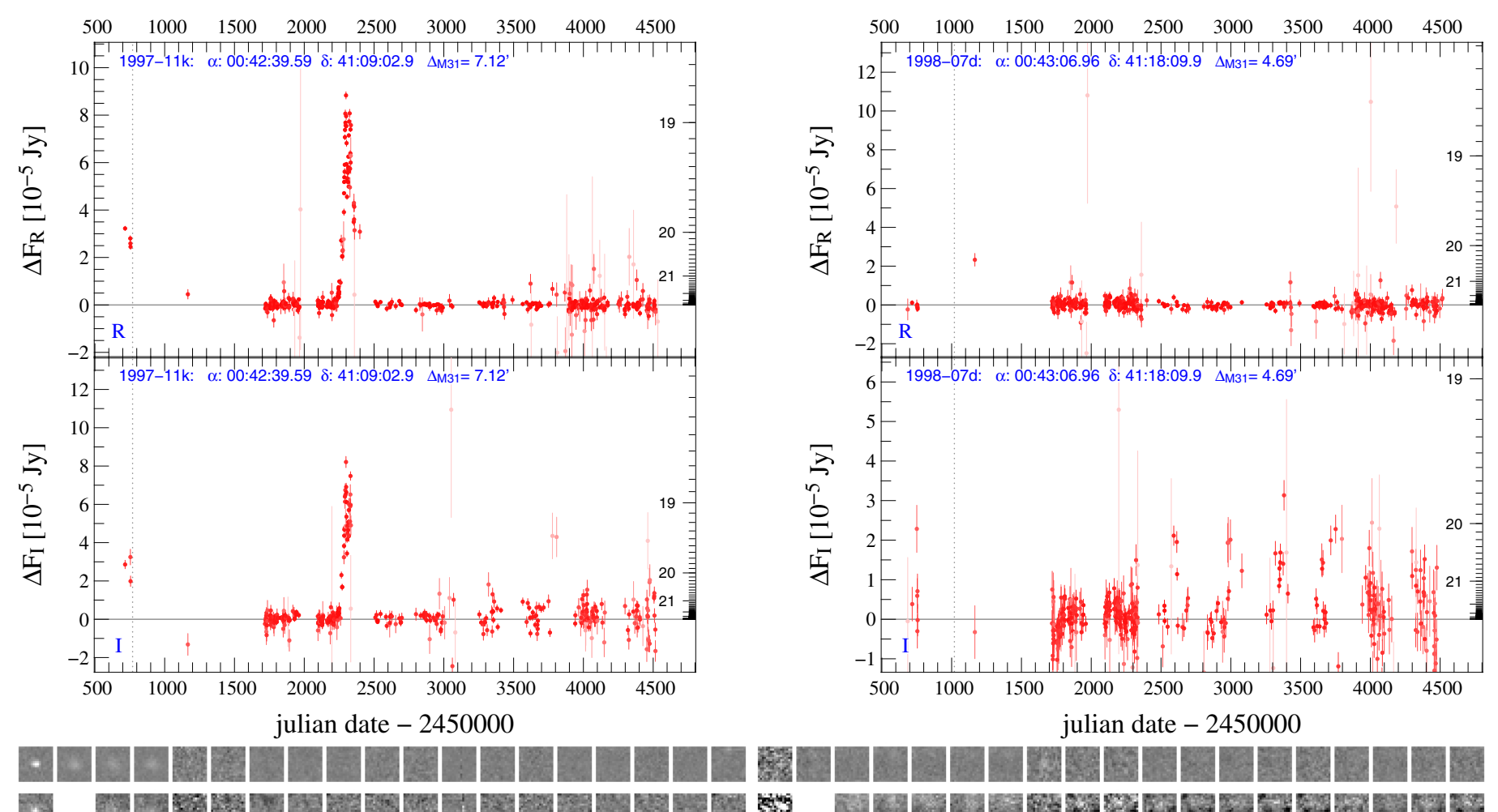

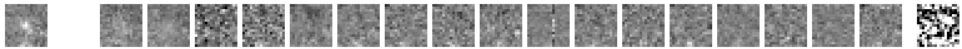
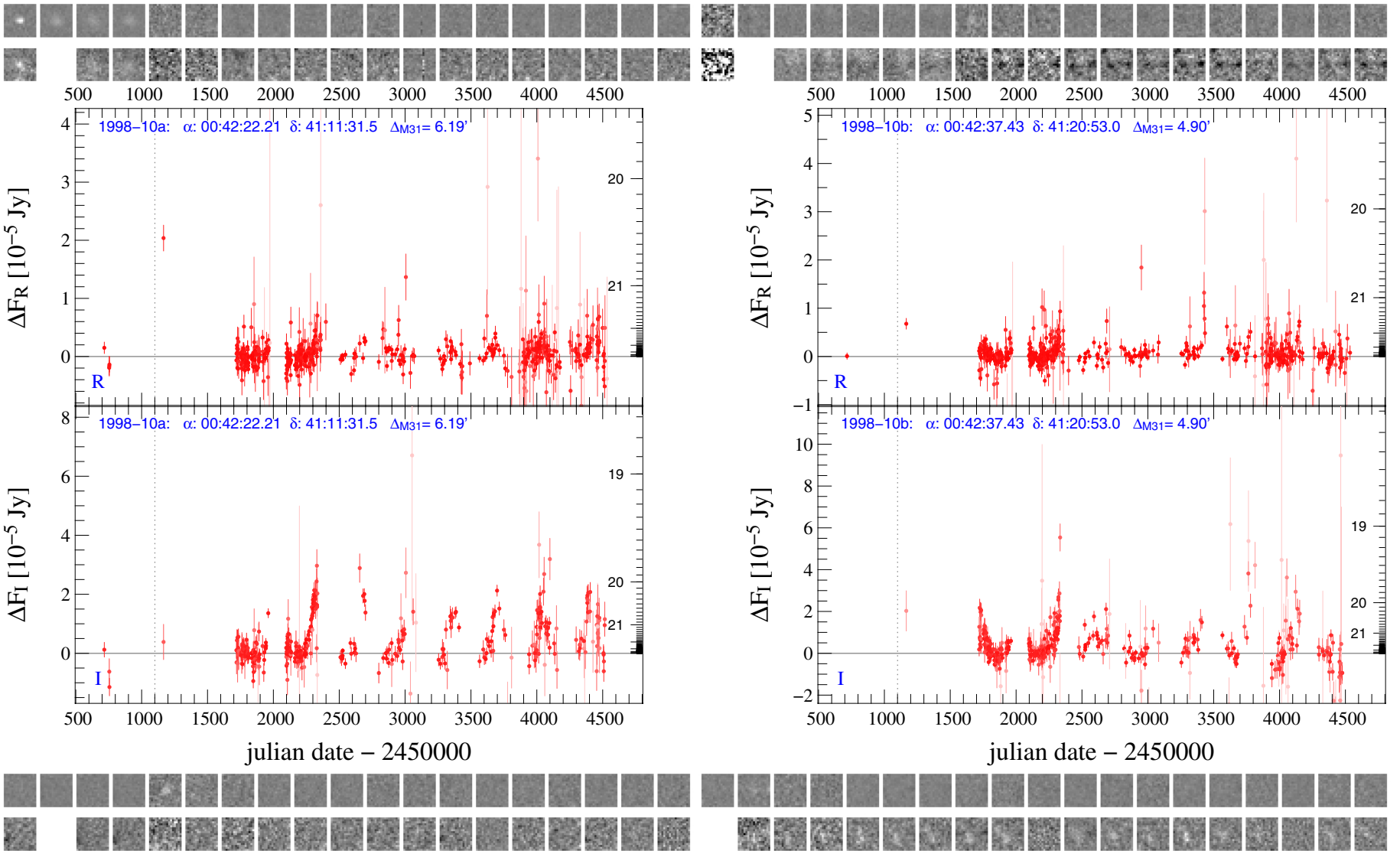

Fig. B.2. Light curve of 1997-11k, 1998-07d, 1998-10a, and 1998-10b from WeCAPP data. 
C.-H. Lee et al.: The Wendelstein Calar Alto Pixellensing Project (WeCAPP): the M 31 nova catalogue
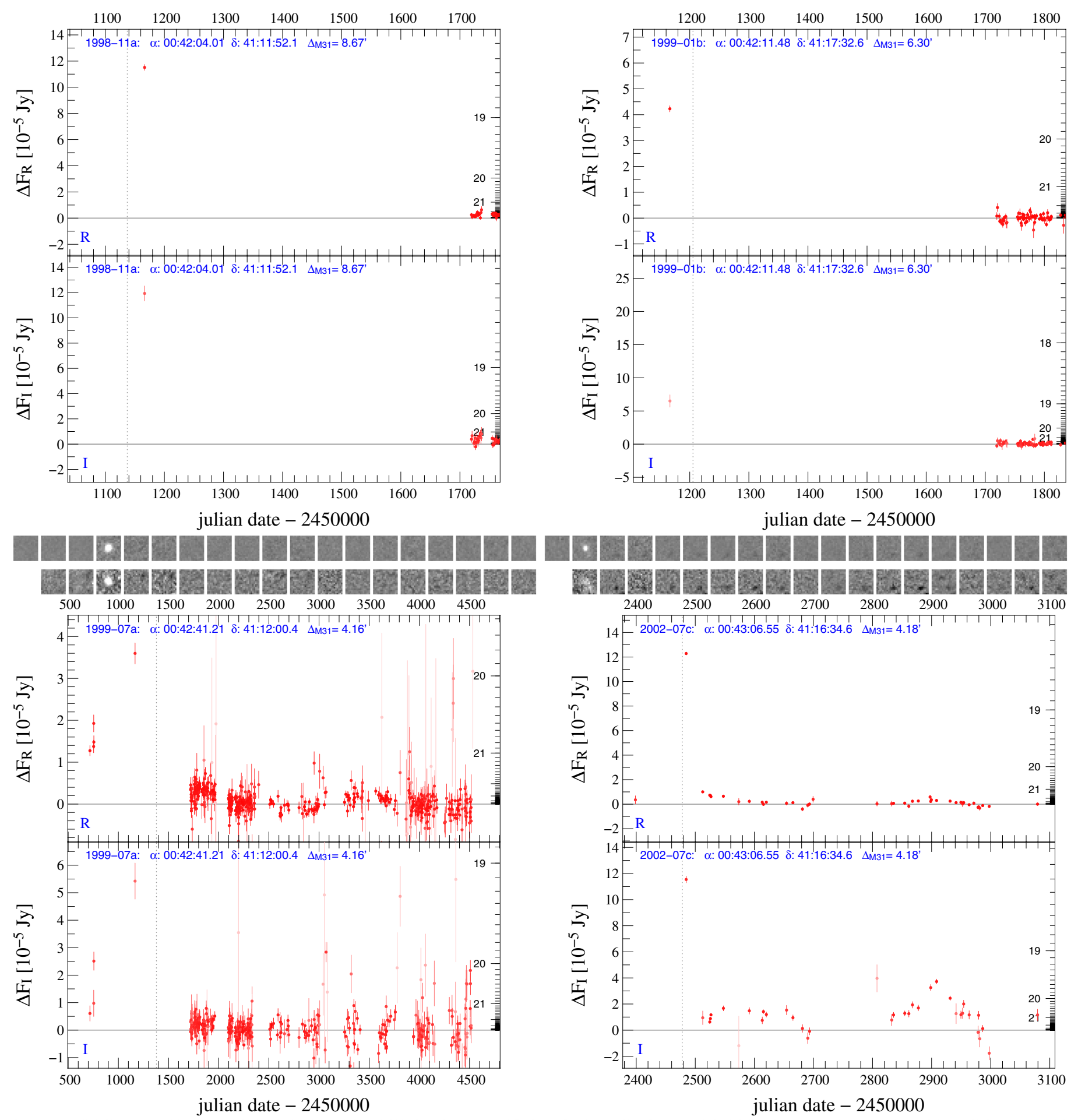

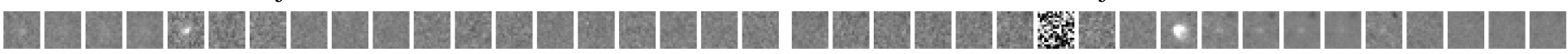

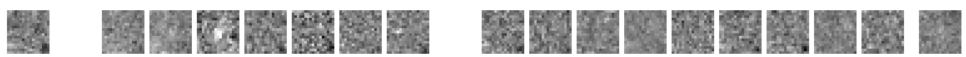

Fig. B.3. Light curve of 1998-11a, 1999-01b, 1999-07a, and 2002-07c from WeCAPP data. 
A\&A 537, A43 (2012)
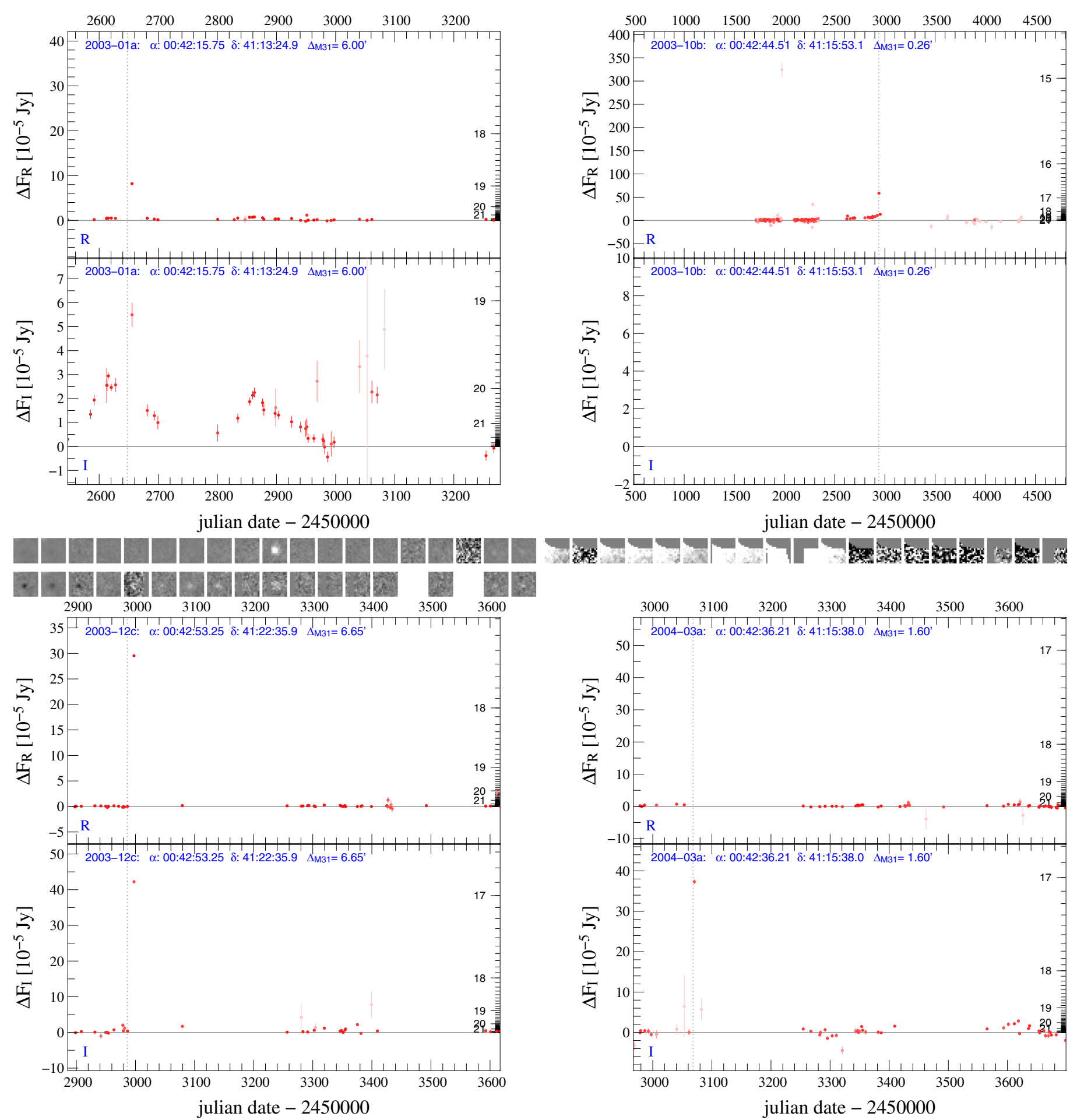

W

Fig. B.4. Light curve of 2003-01a, 2003-10b, 2003-12c, and 2004-03a from WeCAPP data. 
C.-H. Lee et al.: The Wendelstein Calar Alto Pixellensing Project (WeCAPP): the M 31 nova catalogue
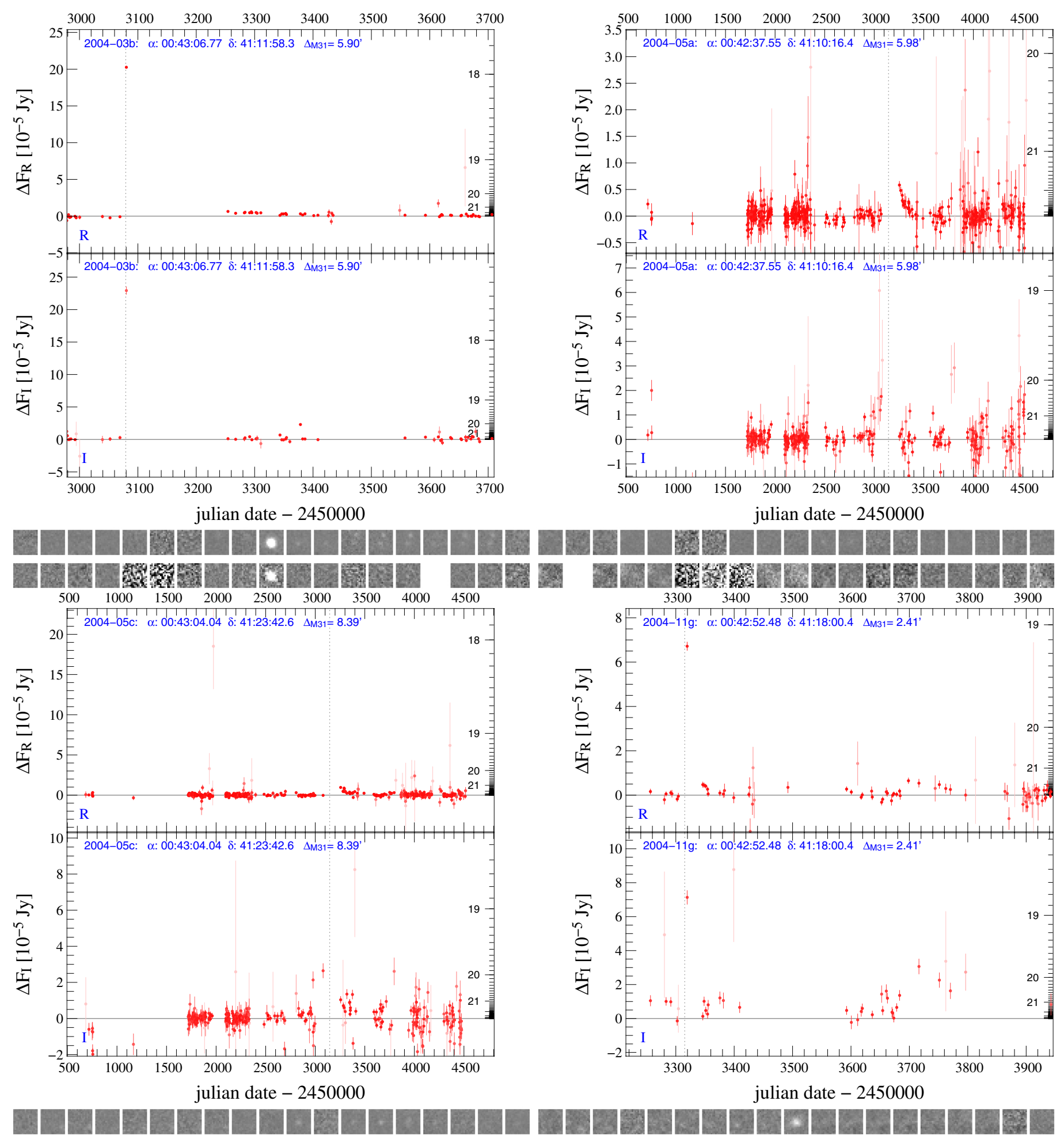

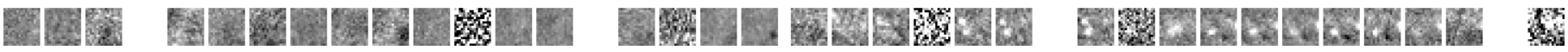

Fig. B.5. Light curve of 2004-03b, 2004-05a, 2004-05c, and 2004-11g from WeCAPP data. 
A\&A 537, A43 (2012)
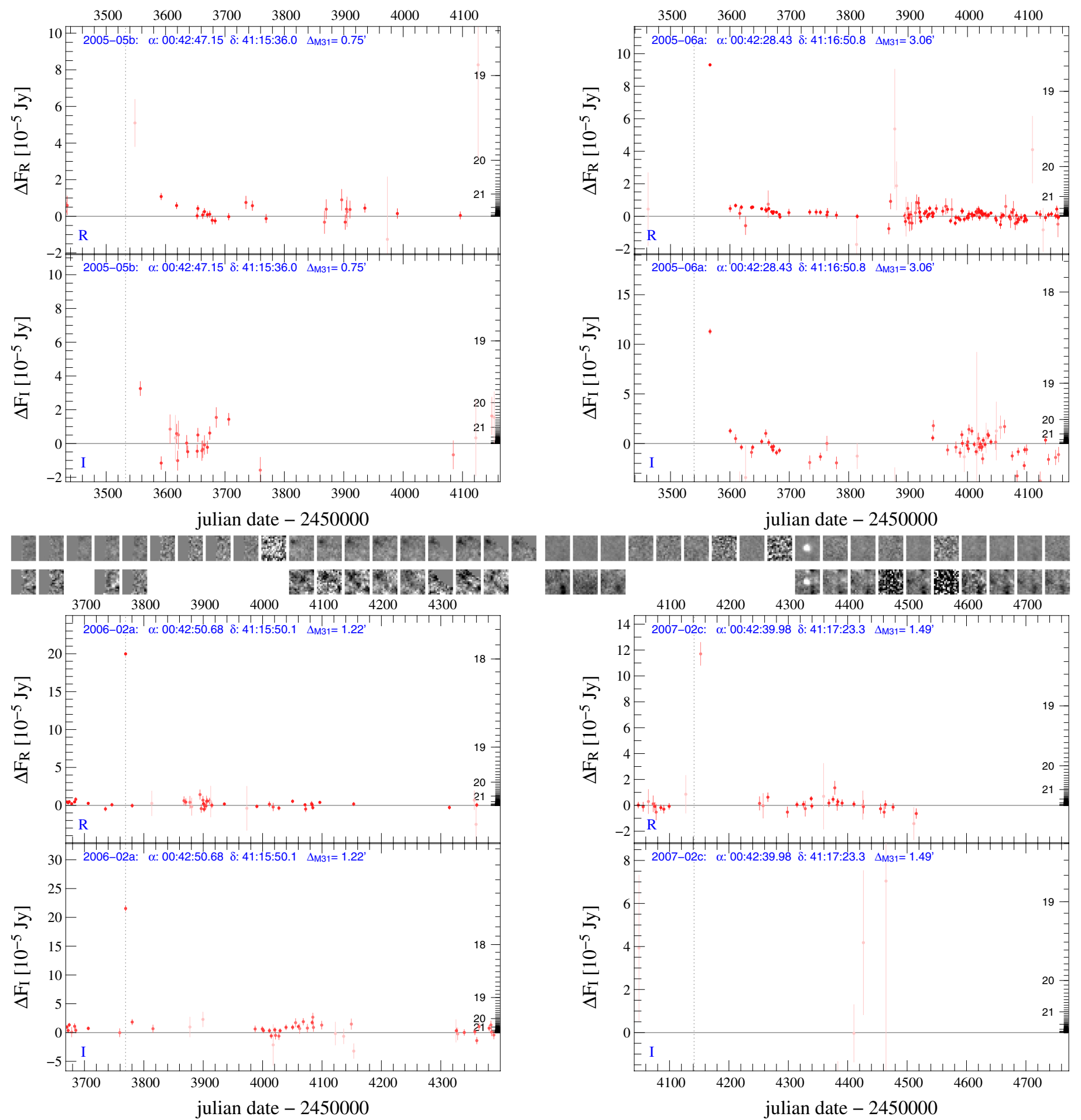

10. R.

Fig. B.6. Light curve of 2005-05b, 22005-06a, 2006-02a, and 2007-02c from WeCAPP data. 
C.-H. Lee et al.: The Wendelstein Calar Alto Pixellensing Project (WeCAPP): the M 31 nova catalogue
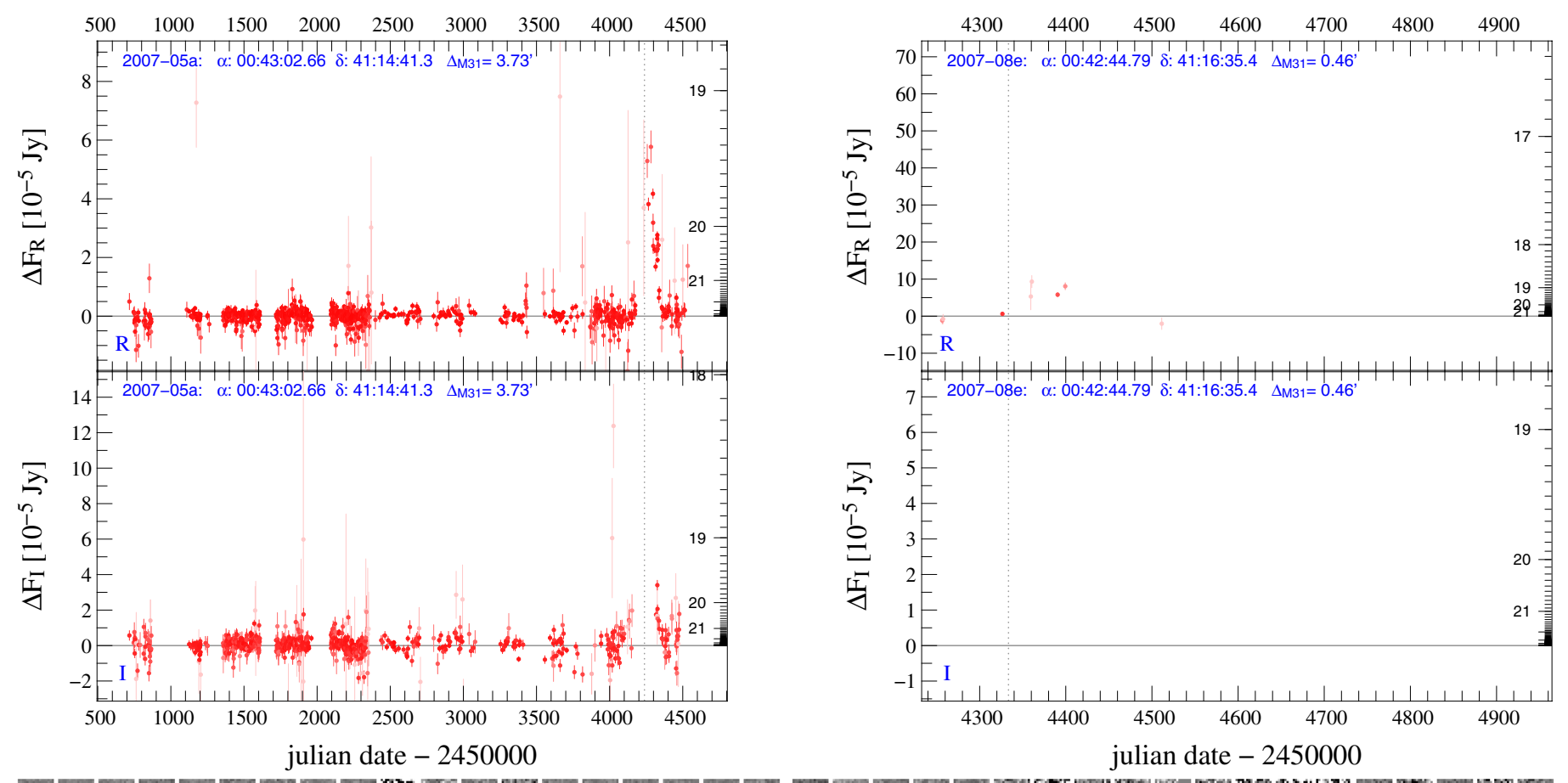

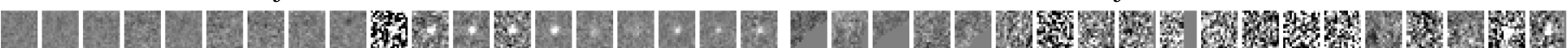

전전
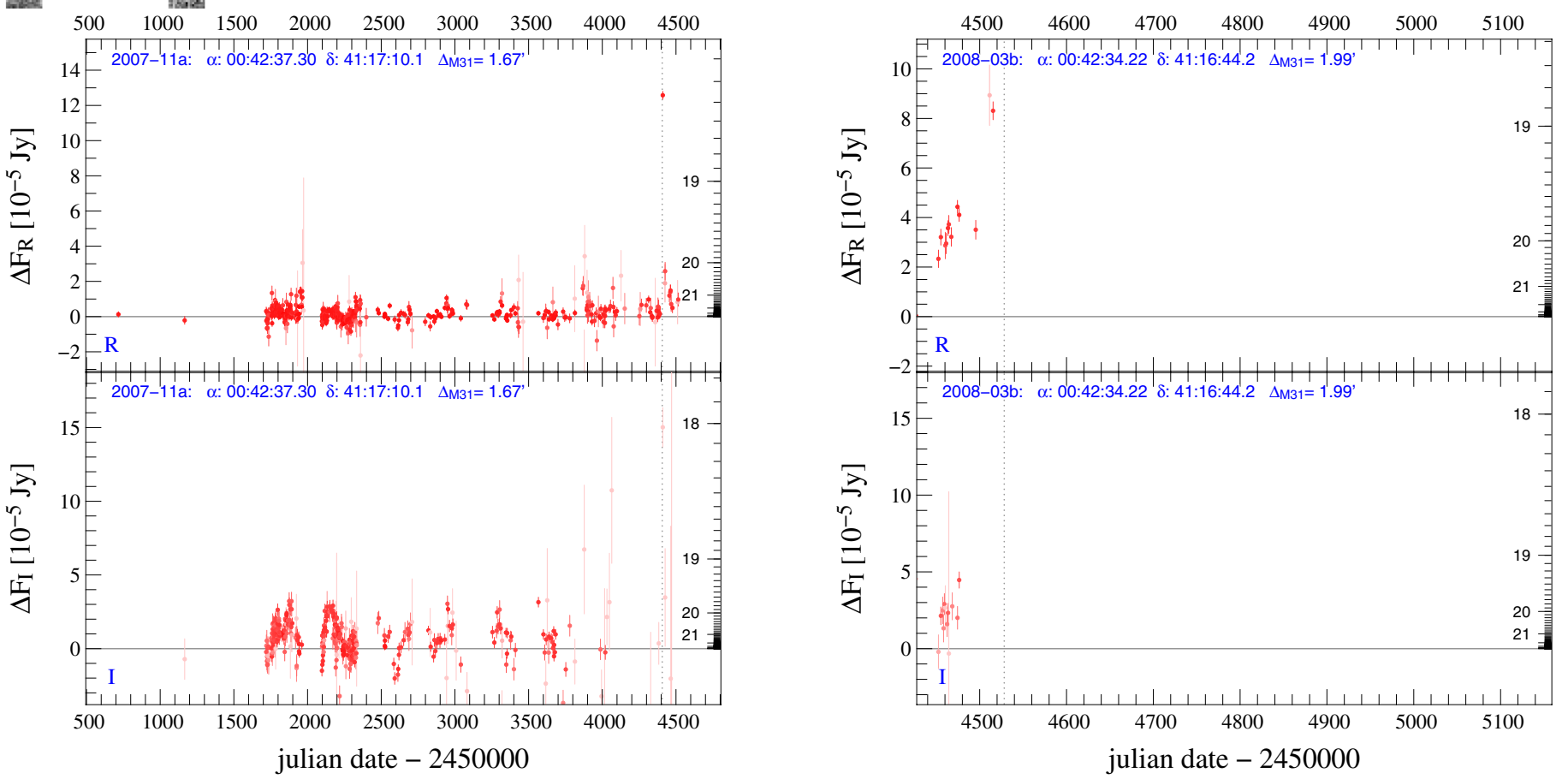

W

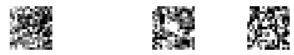

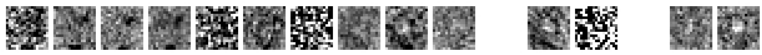

Fig. B.7. Light curve of 2007-05a, 2007-08e, 2007-11a, and 2008-03b from WeCAPP data. 


\section{B.2. Previously reported but doubtful nova candidates}

Fifteen of them are very likely to be variable stars and are shown below. For four (2004-06a, 2004-06b, 2004-06c, and 2005-06c) of them the light curves on which the nova classification is based has been published by Hornoch (see Table B.2 of Pietsch et al. 2007). These published light curves show bright $(<18$ mag in $R$ ) variable sources with nova-like light curves. In all 15 cases in this paragraph (Appendix B.2) we identify semi-periodic variables at the location of the published novae. These variables actually look fairly similar to each other and could form one class of variable objects. It seems unlikely that in each of these cases a nova is located close to the variable without being physically associated. Alternatively these stars could be variables that from time to time have a nova-like outburst. 
C.-H. Lee et al.: The Wendelstein Calar Alto Pixellensing Project (WeCAPP): the M 31 nova catalogue
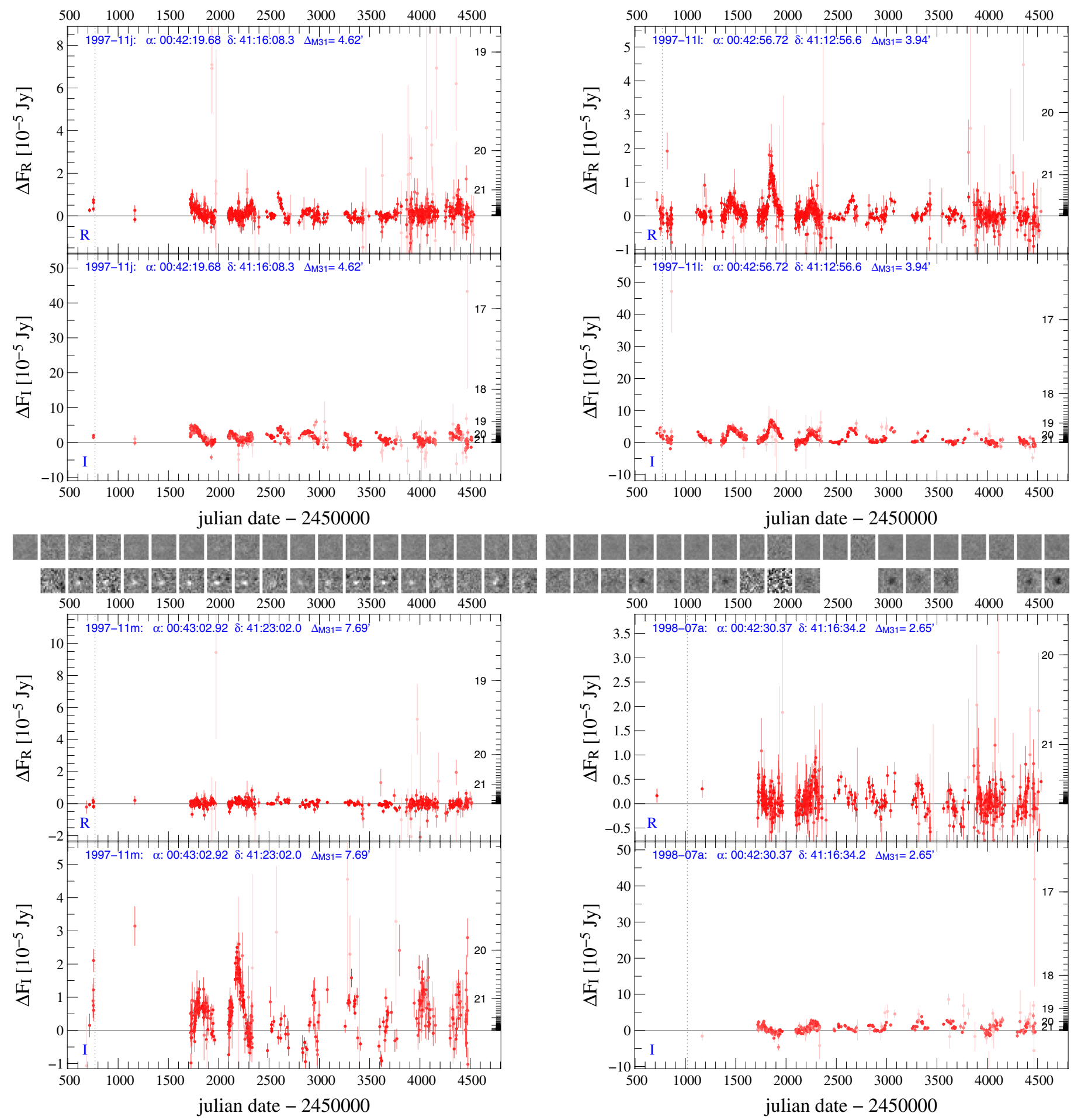

载

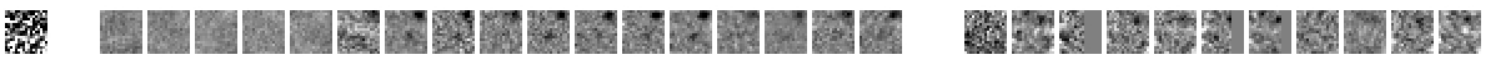

Fig. B.8. Light curve of 1997-11j, 1997-111, 1997-11m, and 1998-07a from WeCAPP data. 
A\&A 537, A43 (2012)
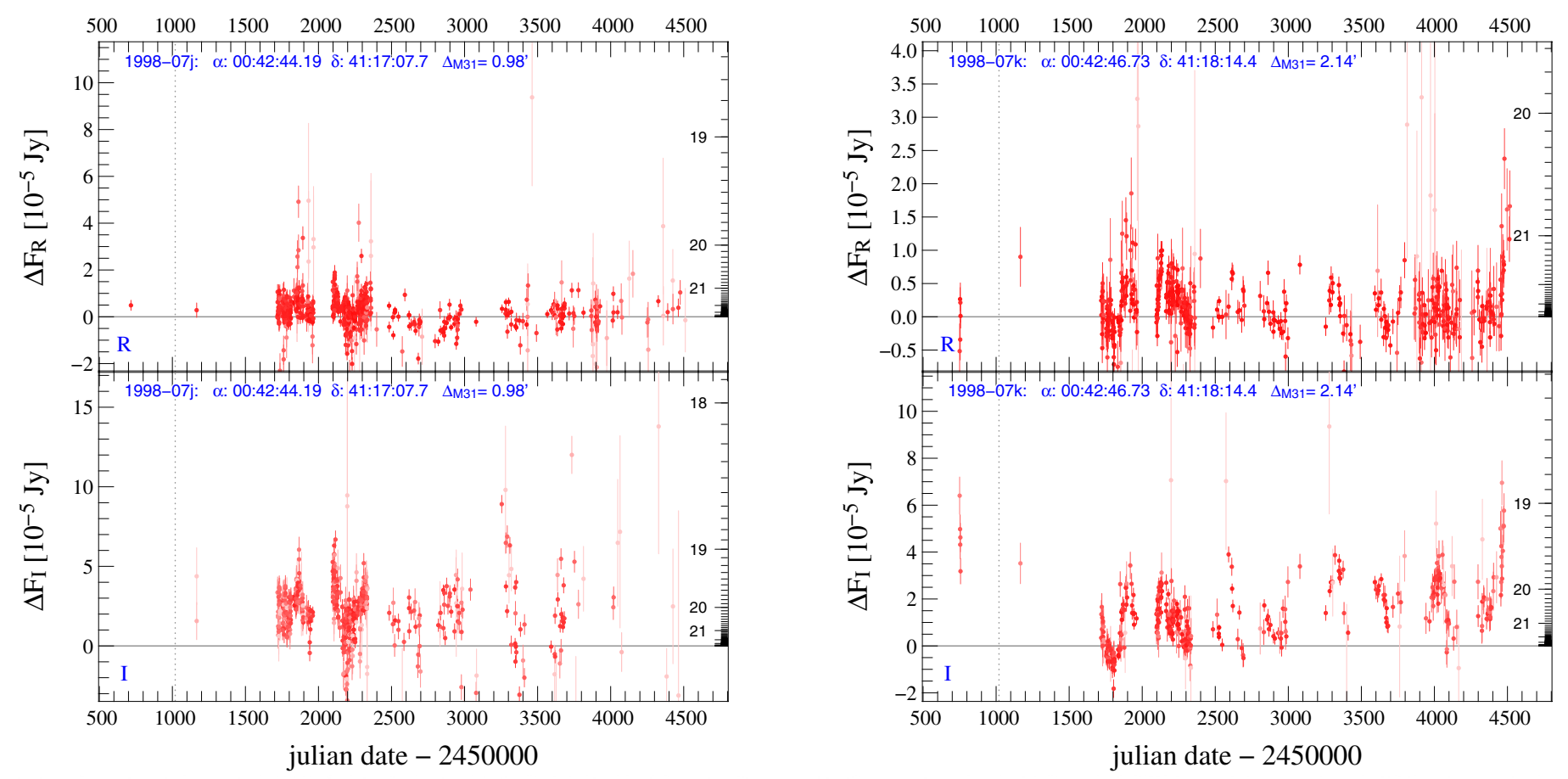

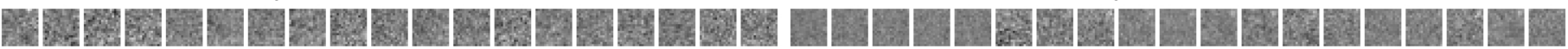

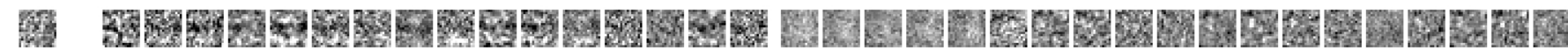
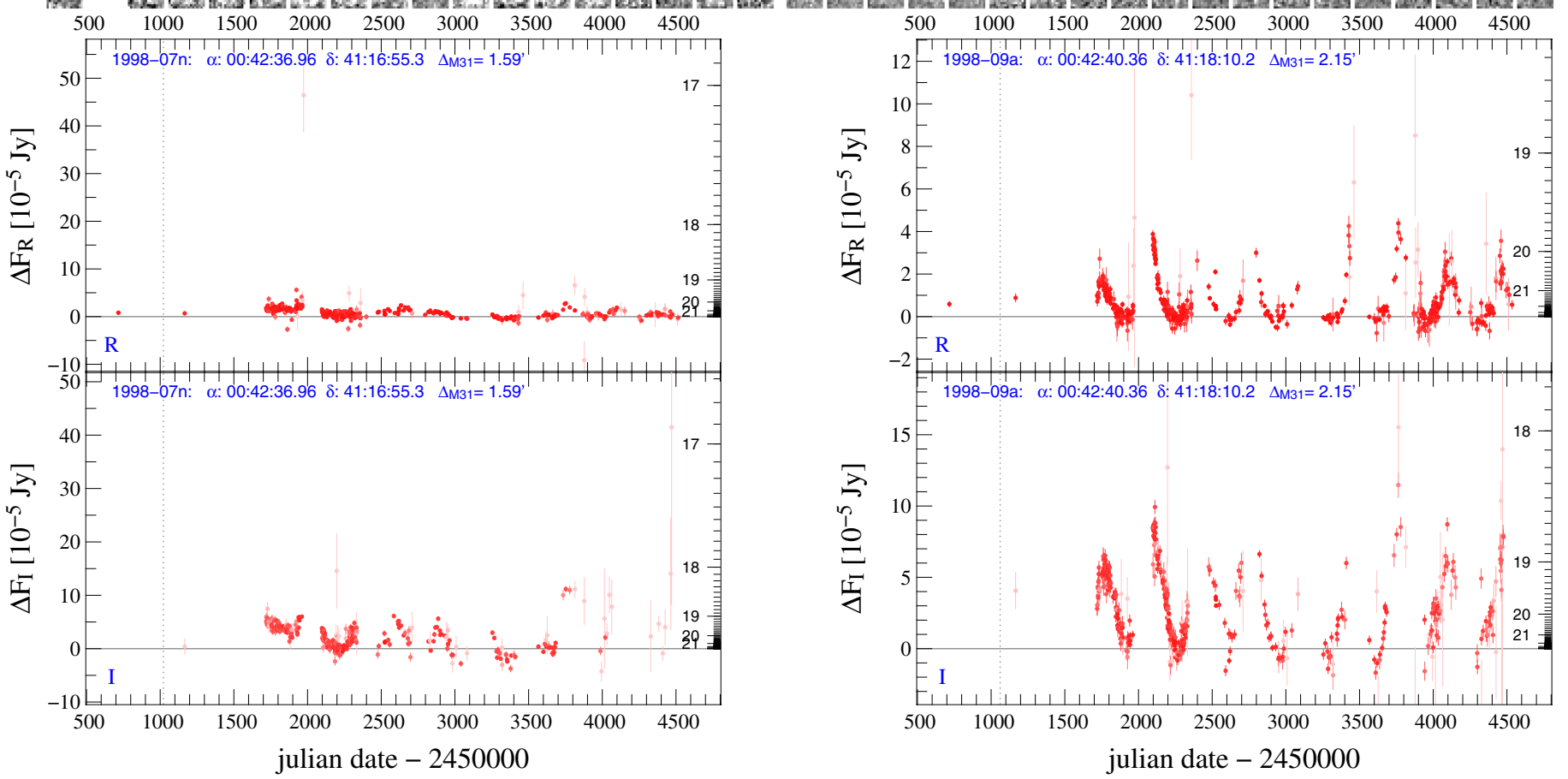

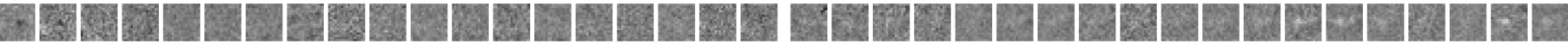

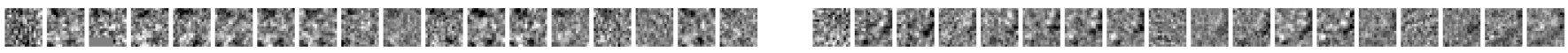

Fig. B.9. Light curve of 1998-07j, 1998-07k, 1998-07n, and 1998-09a from WeCAPP data. 
C.-H. Lee et al.: The Wendelstein Calar Alto Pixellensing Project (WeCAPP): the M 31 nova catalogue
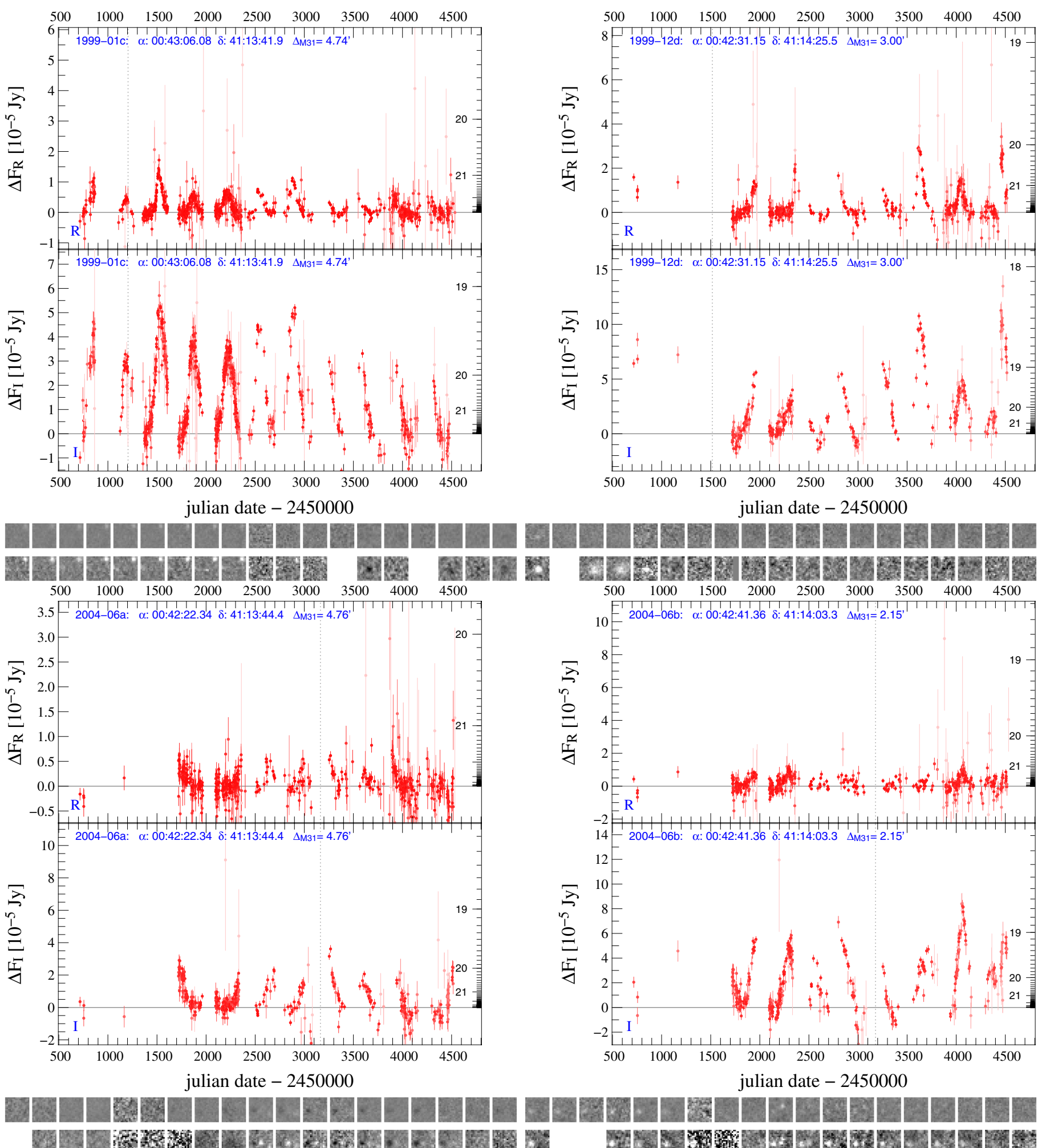

Fig. B.10. Light curve of 1999-01c, 1999-12d, 2004-06a, and 2004-06b from WeCAPP data. 

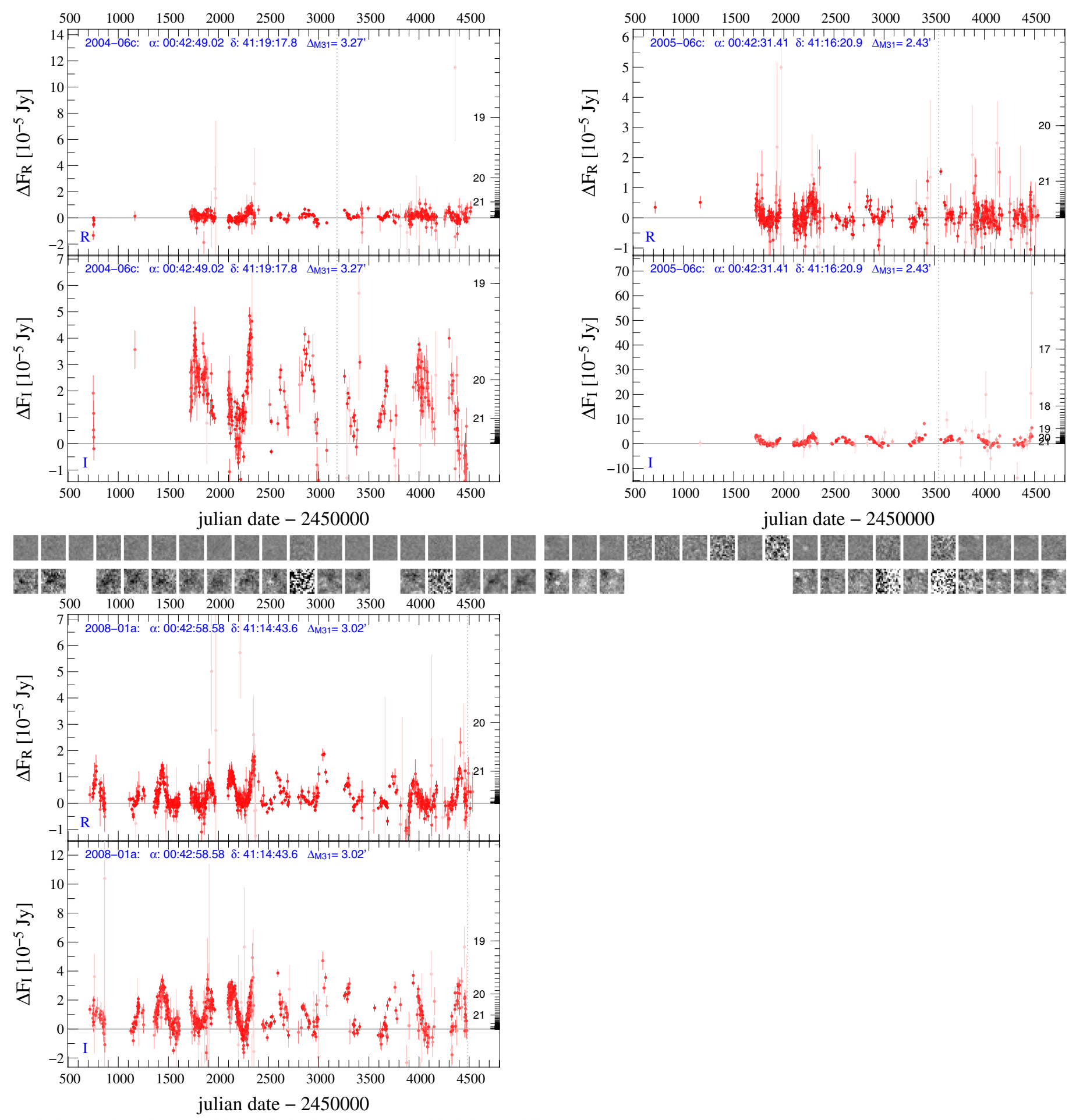

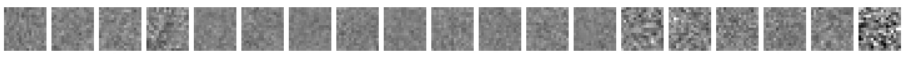

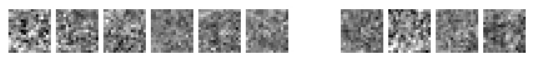

Fig. B.11. Light curve of 2004-06c, 2005-06c, and 2008-01a from WeCAPP data. 
C.-H. Lee et al.: The Wendelstein Calar Alto Pixellensing Project (WeCAPP): the M 31 nova catalogue

For six of these previous nova candidates we see minor variability at the time of nova detection but the light curve has no nova features otherwise.
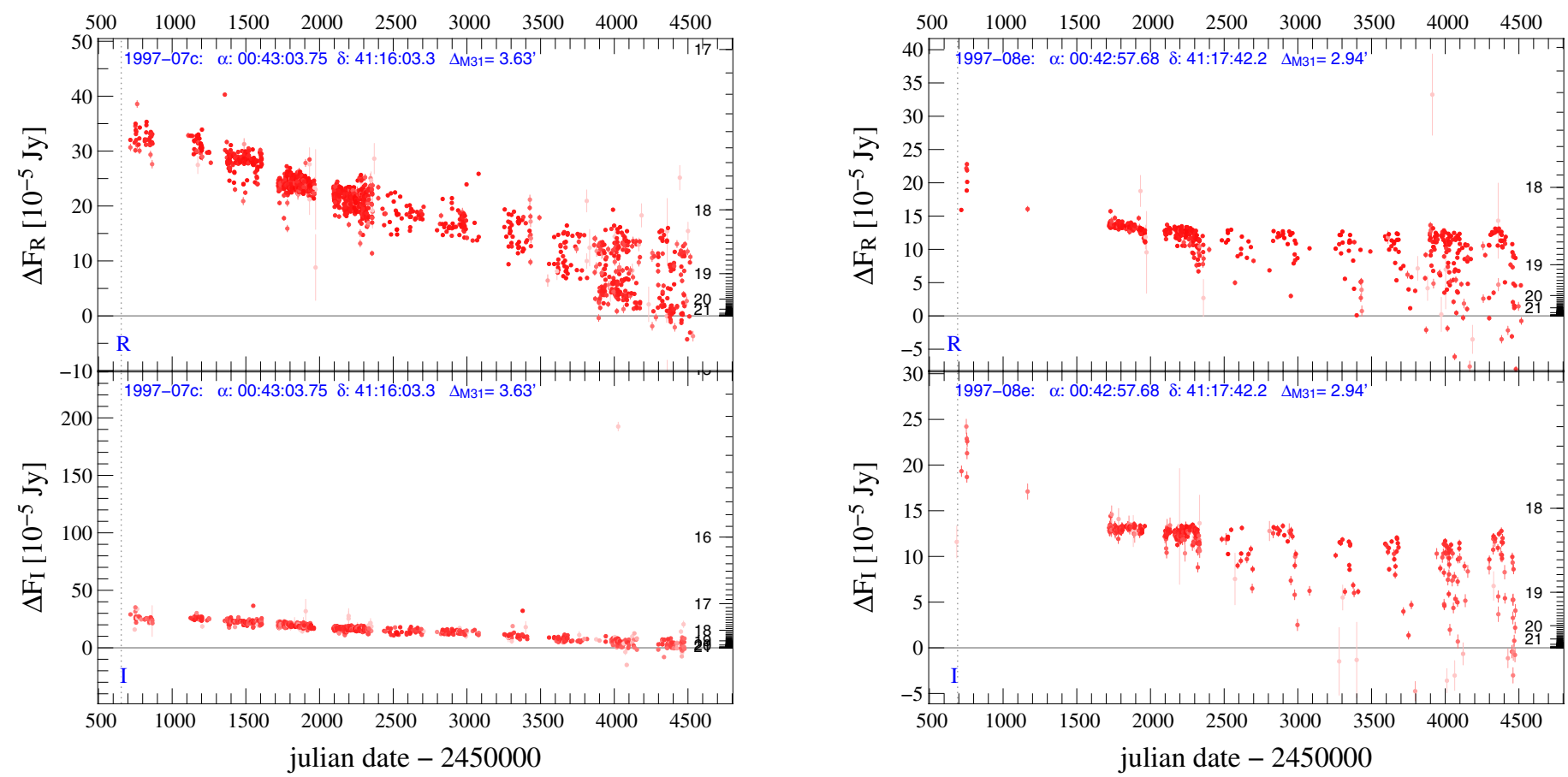

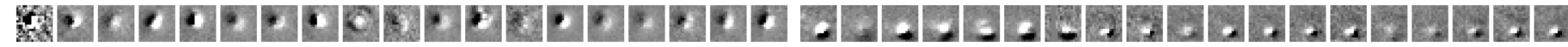
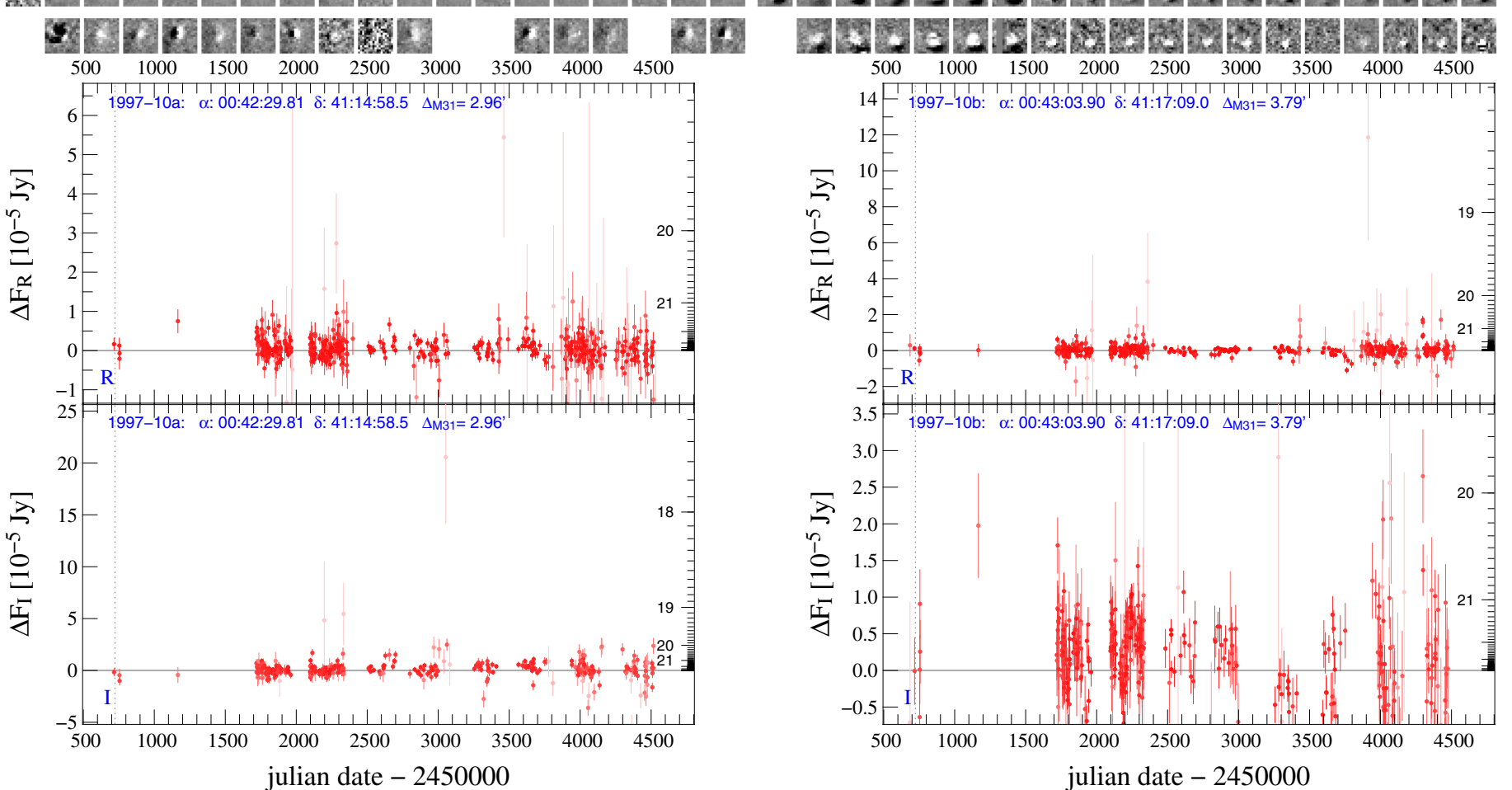

Fig. B.12. Light curve of 1997-07c, 1997-08e, 1997-10a, and 1997-10b from WeCAPP data. 
A\&A 537, A43 (2012)
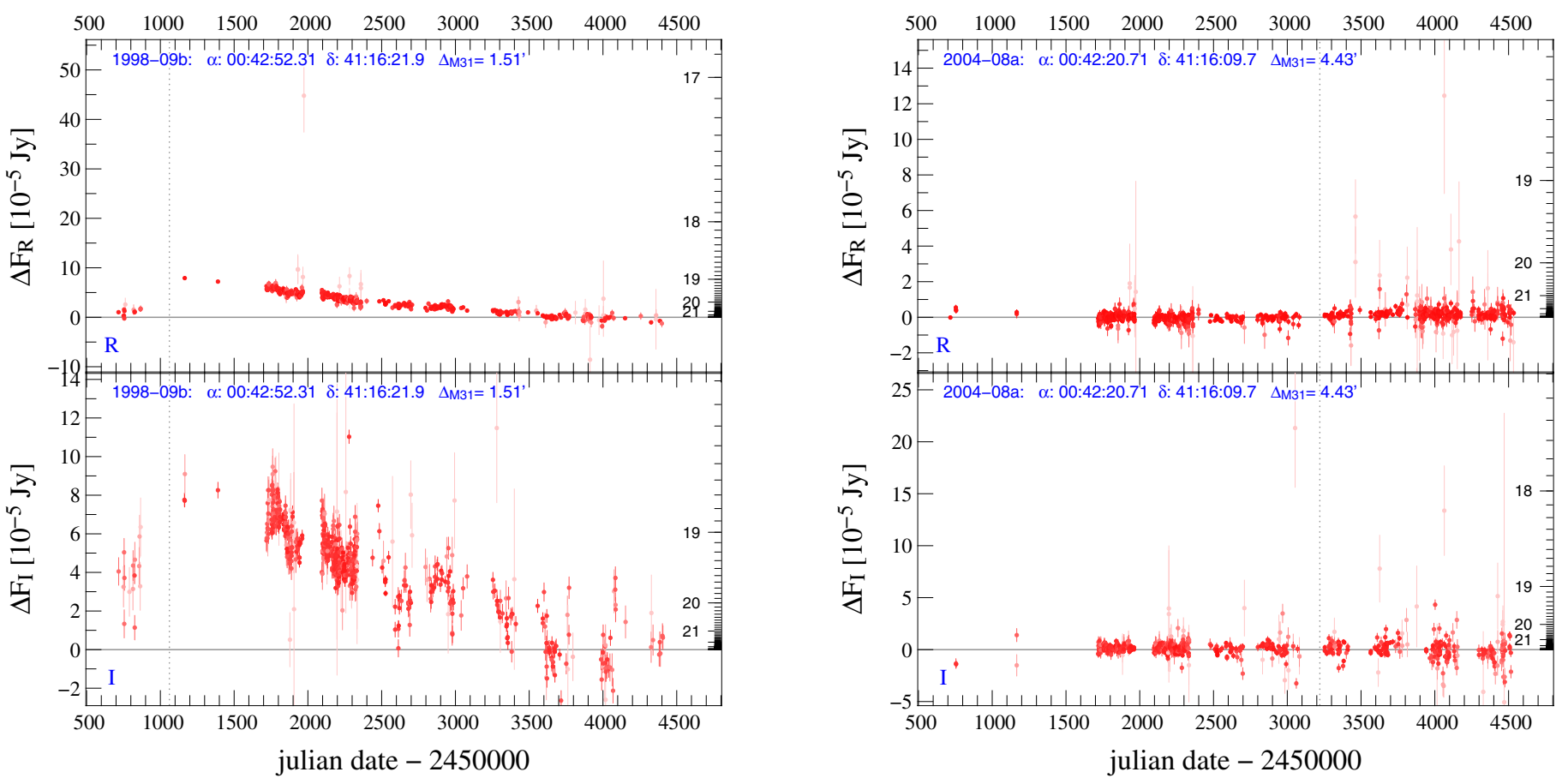

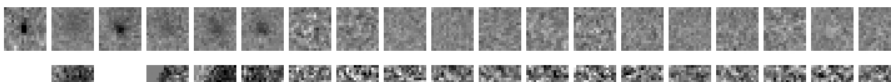

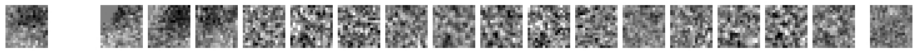

Fig. B.13. Light curve of 1998-09b and 2004-08a from WeCAPP data. 
C.-H. Lee et al.: The Wendelstein Calar Alto Pixellensing Project (WeCAPP): the M 31 nova catalogue

\section{B.3. Nova candidates with no data around the maximum brightness}

For these 26 nova candidates we have no data at all around the maximum brightness so we cannot contribute to the classification of these objects. However, we have data at previous or later times that can be combined with those from the original nova measurements to further constrain the nova nature.
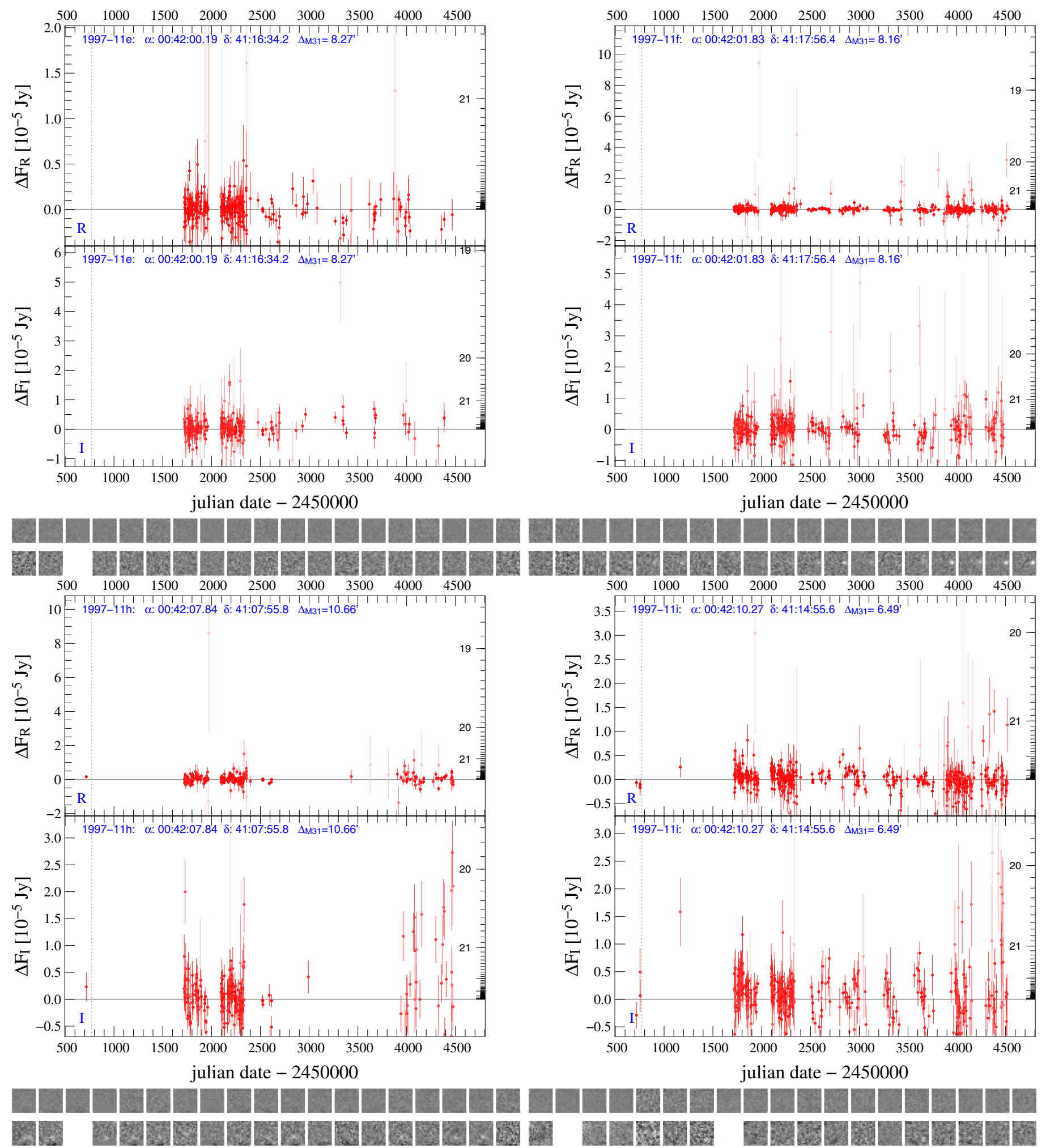

Fig. B.14. Light curve of 1997-11e, 1997-11f, 1997-11h, and 1997-11i from WeCAPP data. 
A\&A 537, A43 (2012)
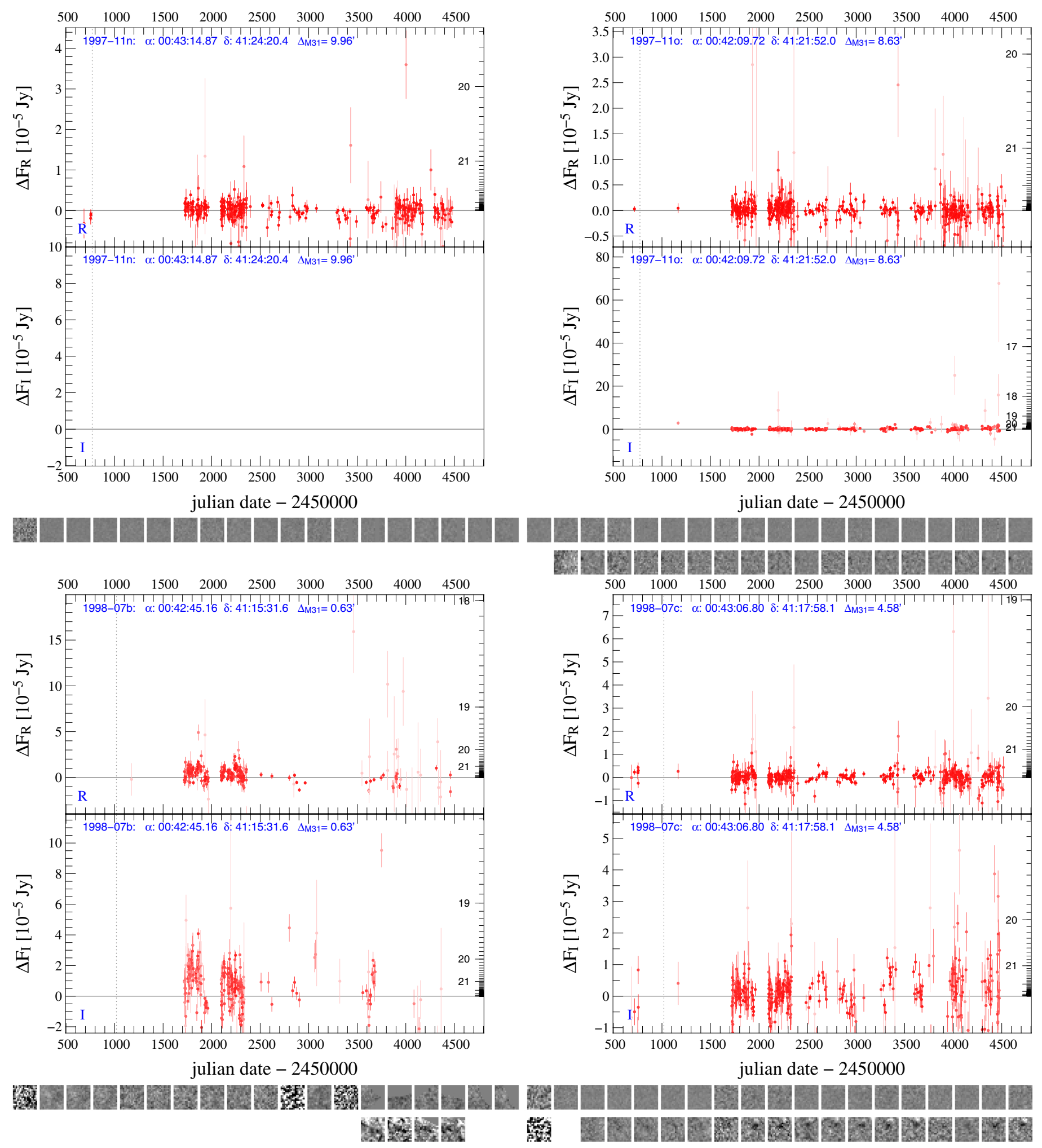

Fig. B.15. Light curve of 1997-11n, 1997-11o, 1998-07b, and 1998-07c from WeCAPP data. 
C.-H. Lee et al.: The Wendelstein Calar Alto Pixellensing Project (WeCAPP): the M 31 nova catalogue
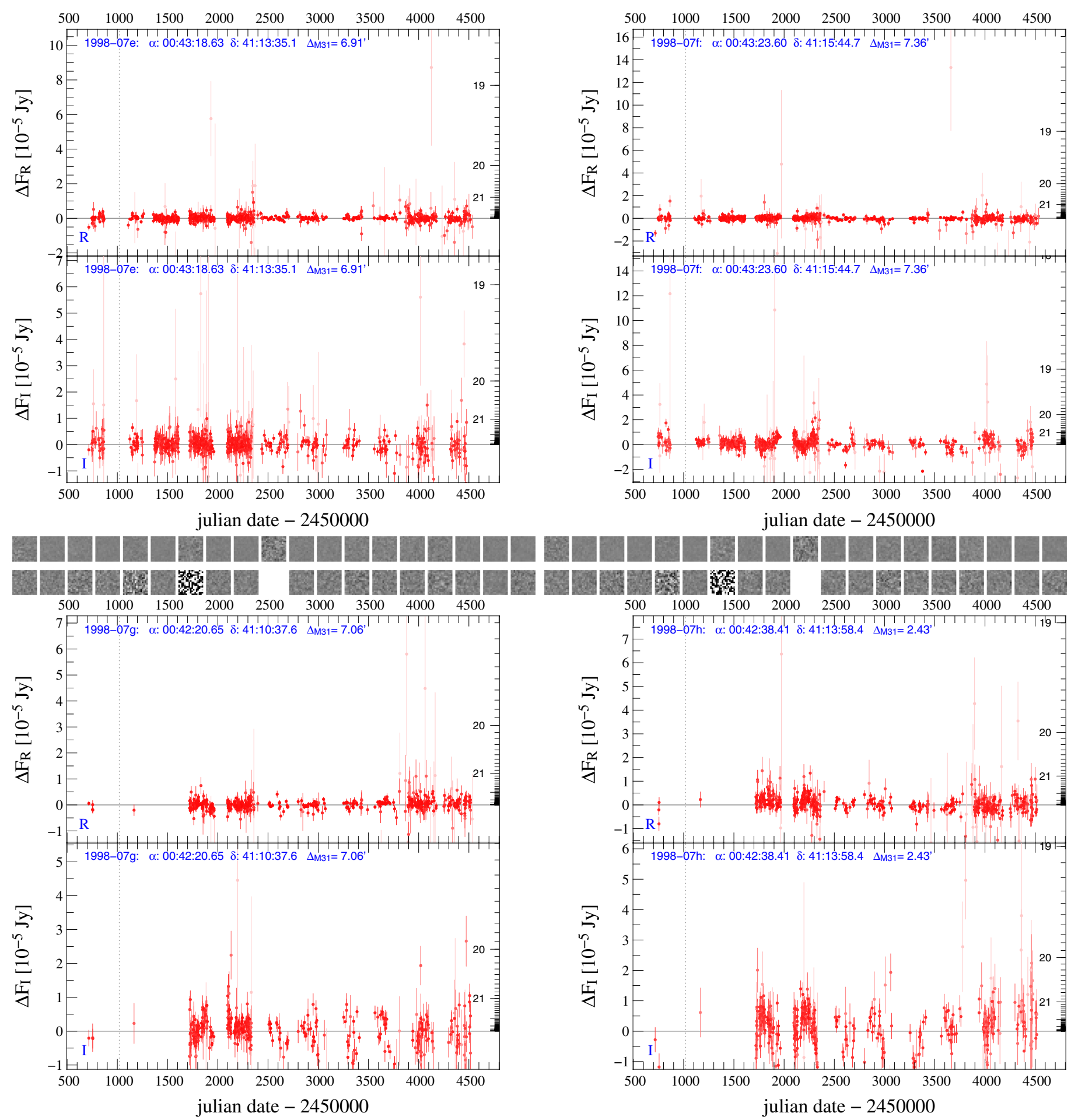

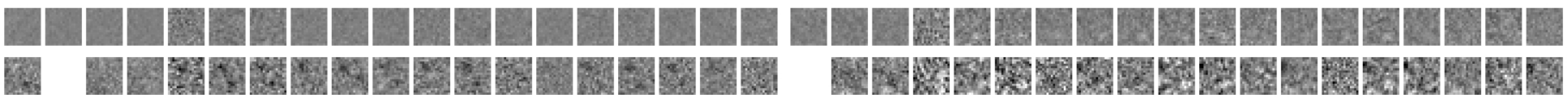

Fig. B.16. Light curve of 1998-07e, 1998-07f, 1998-08g, and 1998-07h from WeCAPP data. 

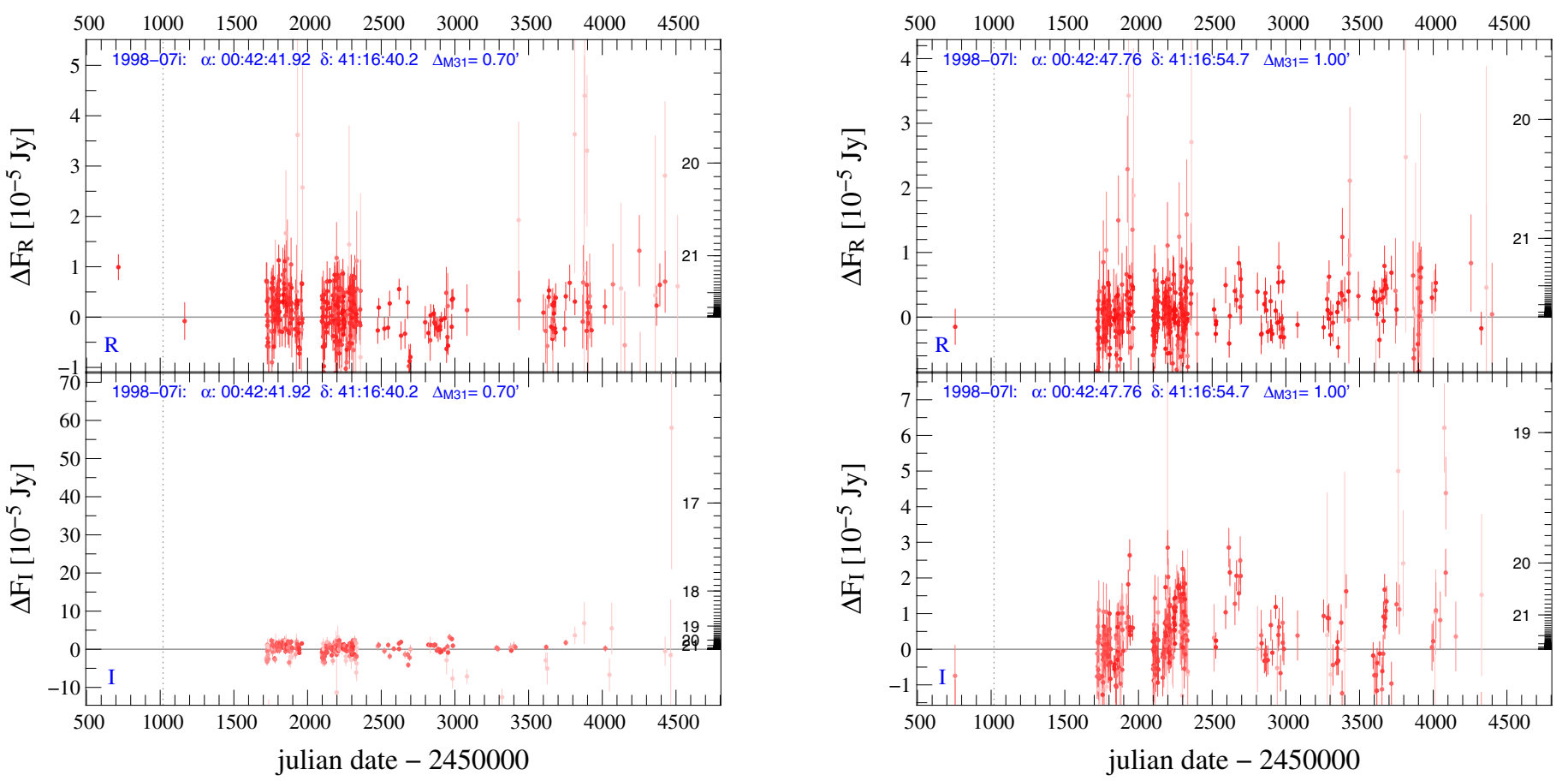

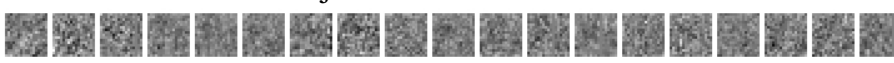

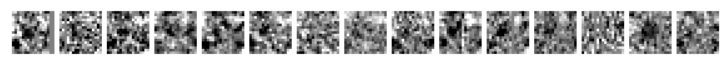

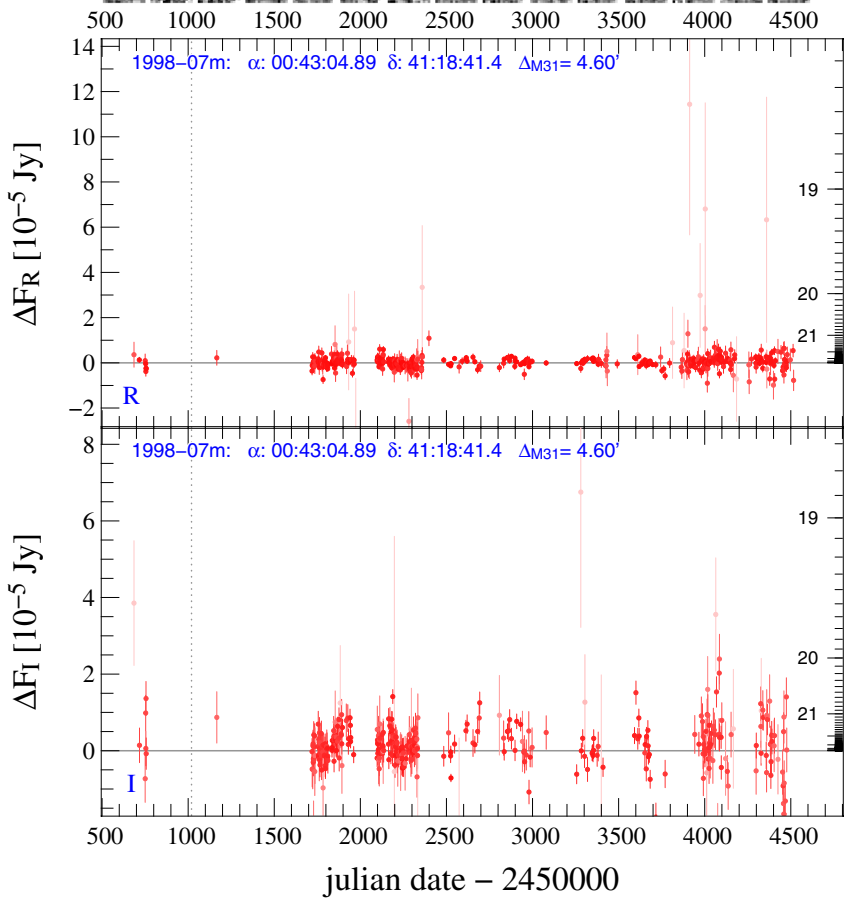

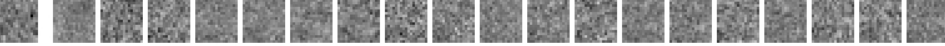

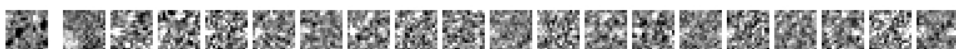

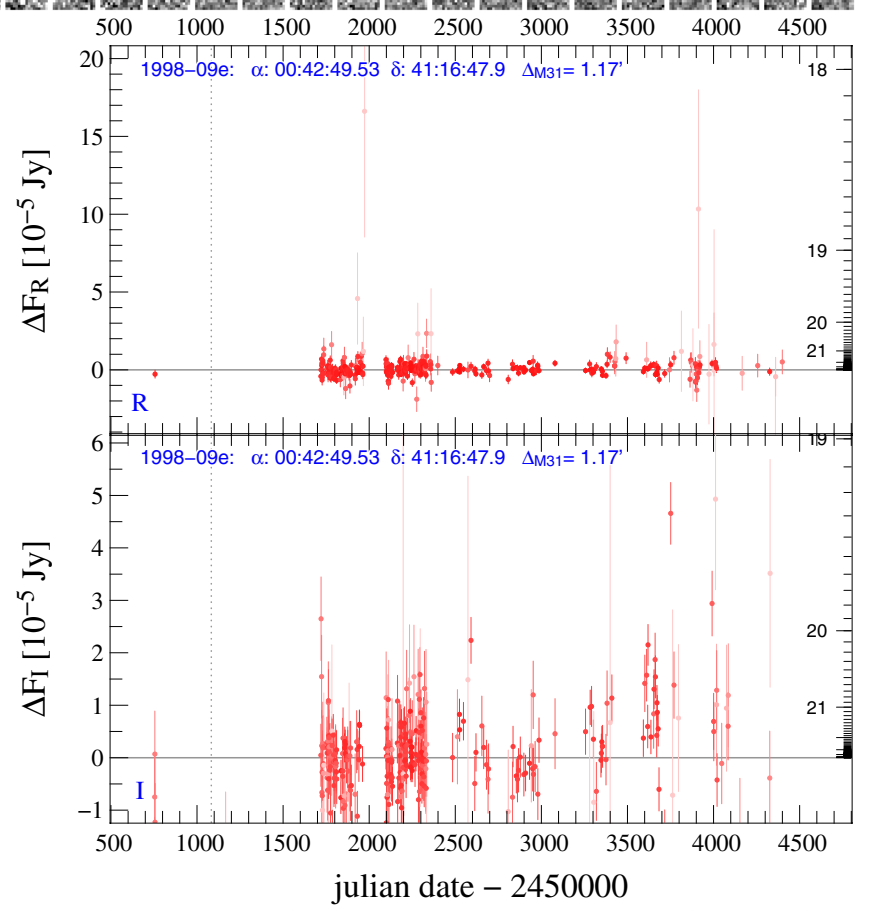

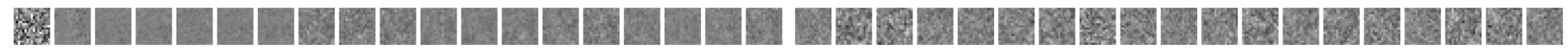

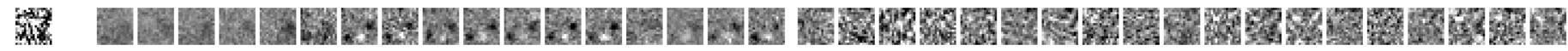

Fig. B.17. Light curve of 1998-07i, 1998-071, 1998-07m, and 1998-09e from WeCAPP data. 
C.-H. Lee et al.: The Wendelstein Calar Alto Pixellensing Project (WeCAPP): the M 31 nova catalogue
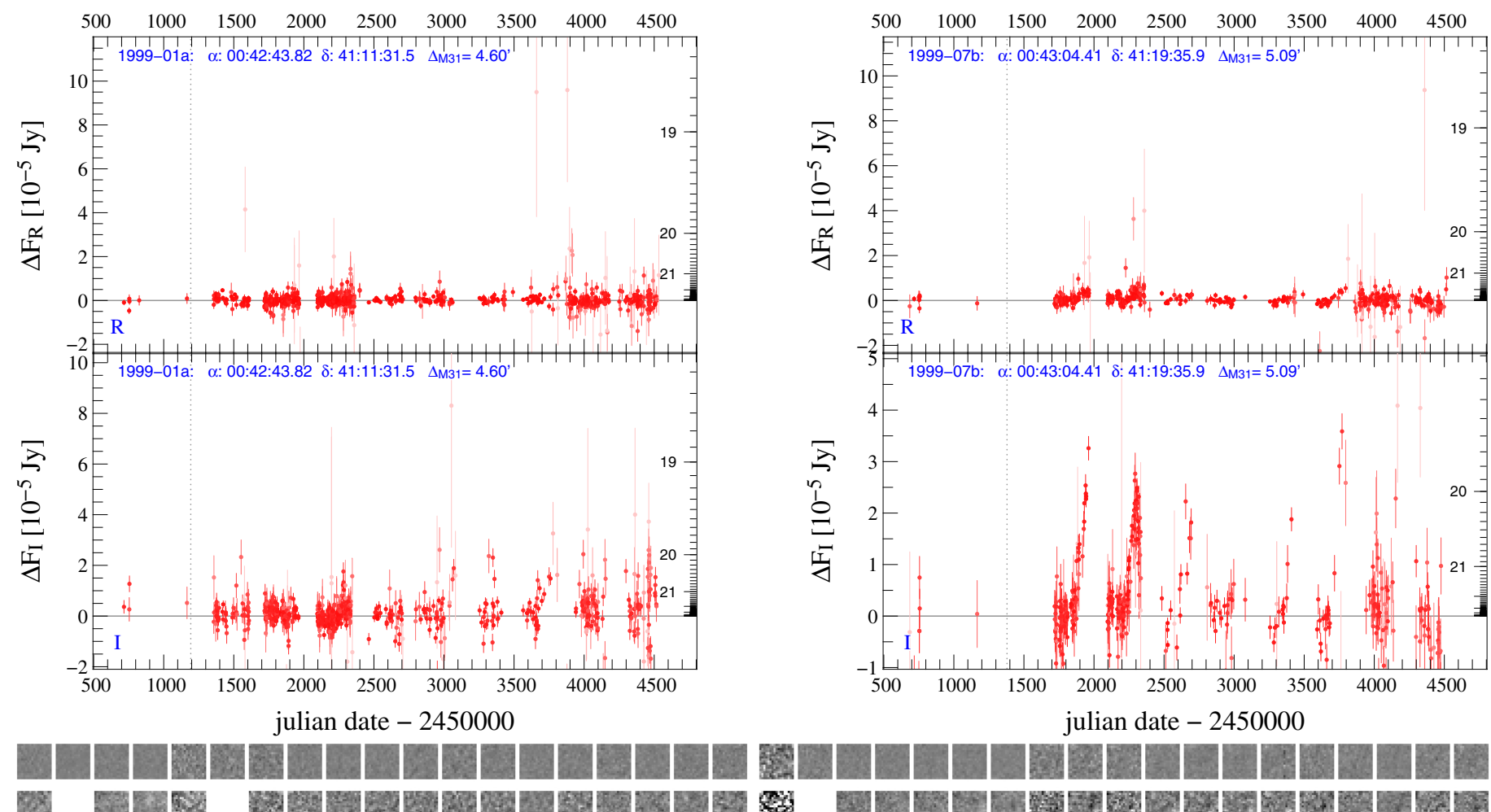

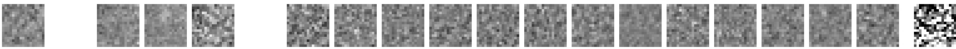
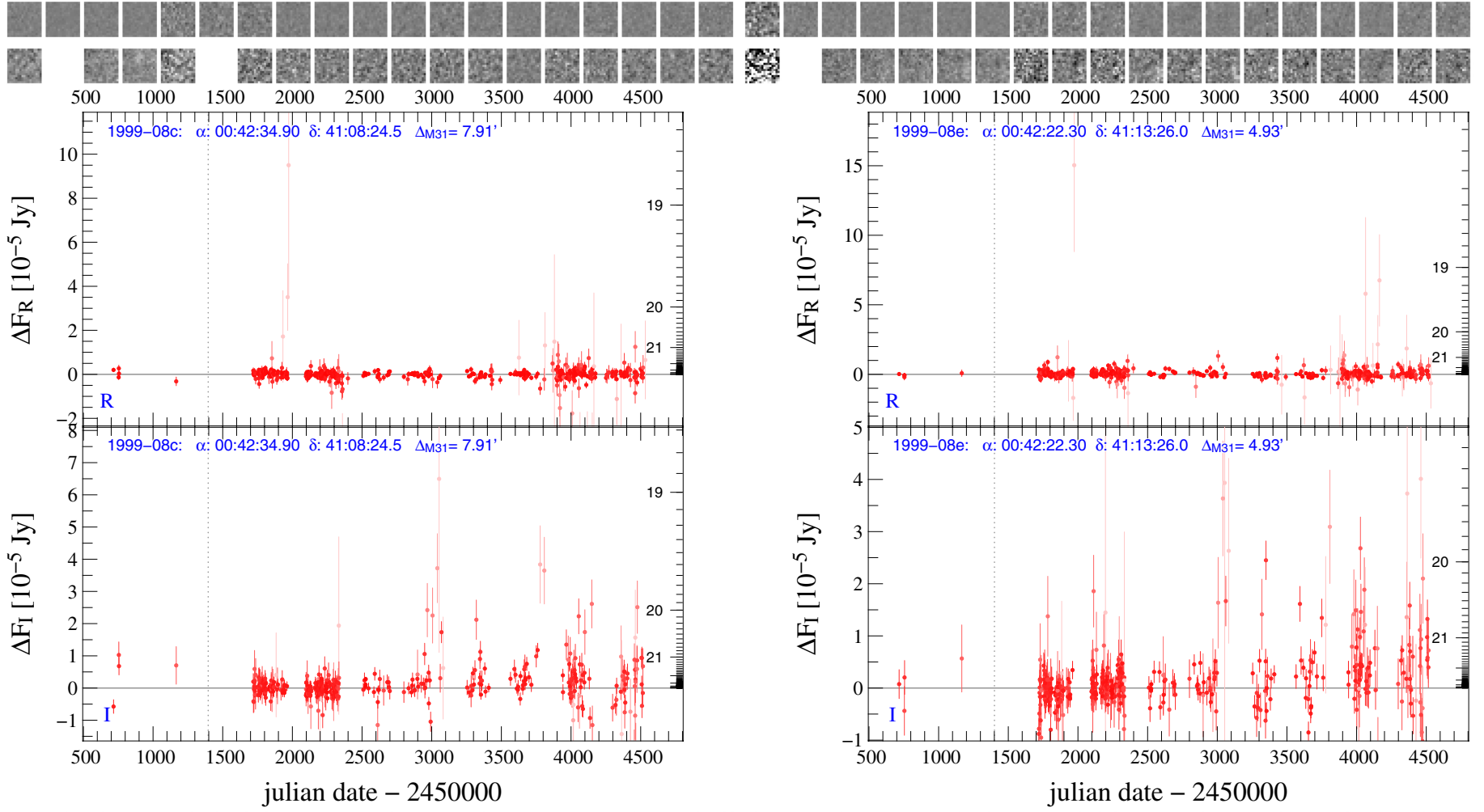

Fig. B.18. Light curve of 1999-01a, 1999-07b, 1999-08c, and 1999-08e from WeCAPP data. 

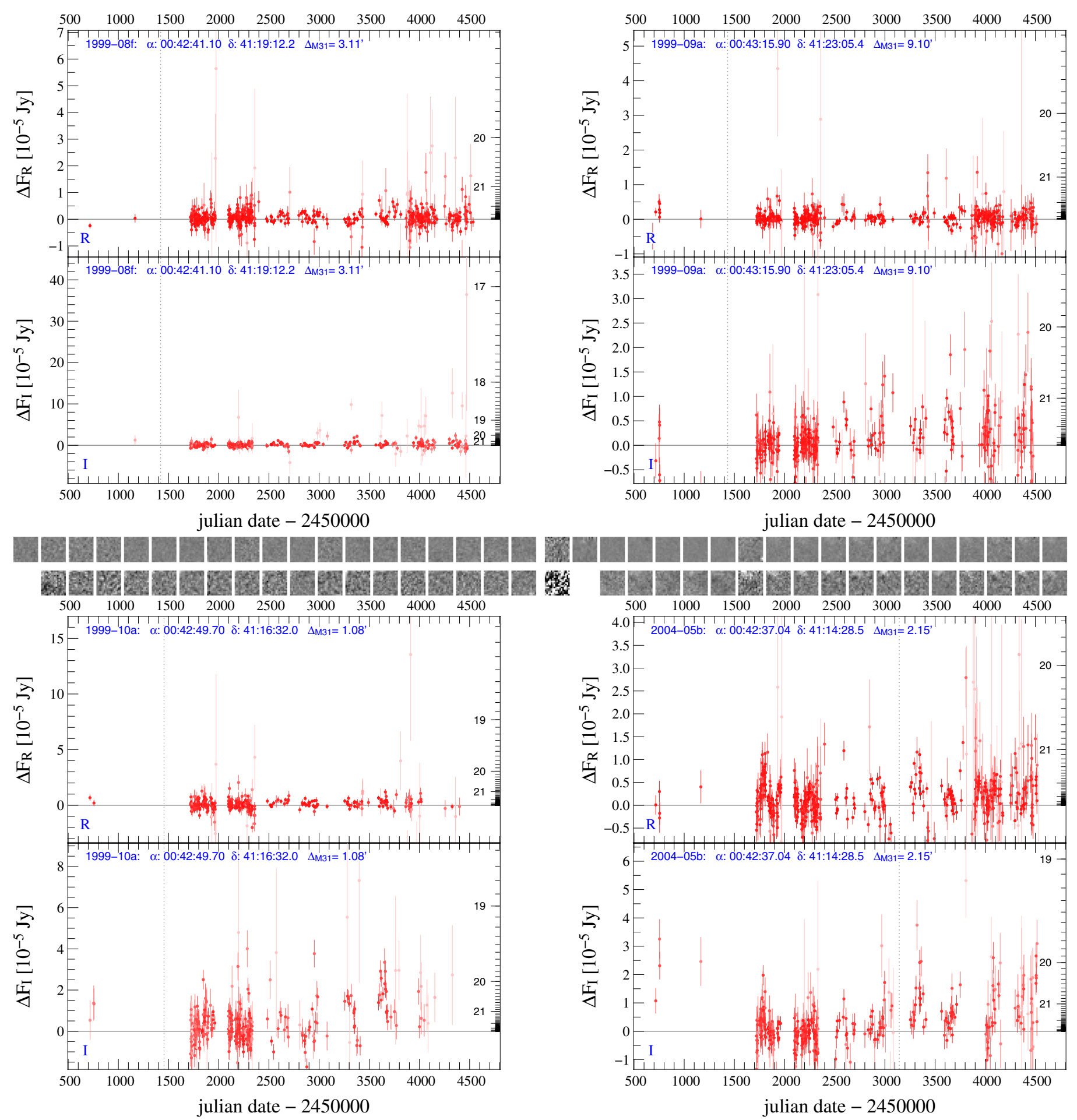

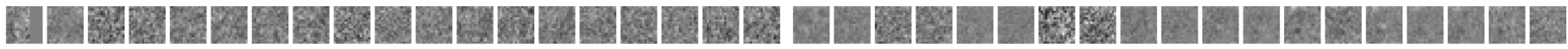

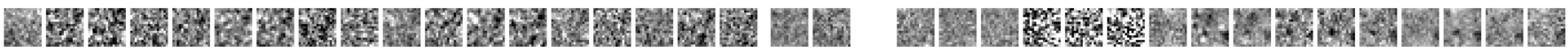

Fig. B.19. Light curve of 1999-08f, 1999-09a, 1999-10a, and 2004-05b from WeCAPP data. 
C.-H. Lee et al.: The Wendelstein Calar Alto Pixellensing Project (WeCAPP): the M 31 nova catalogue
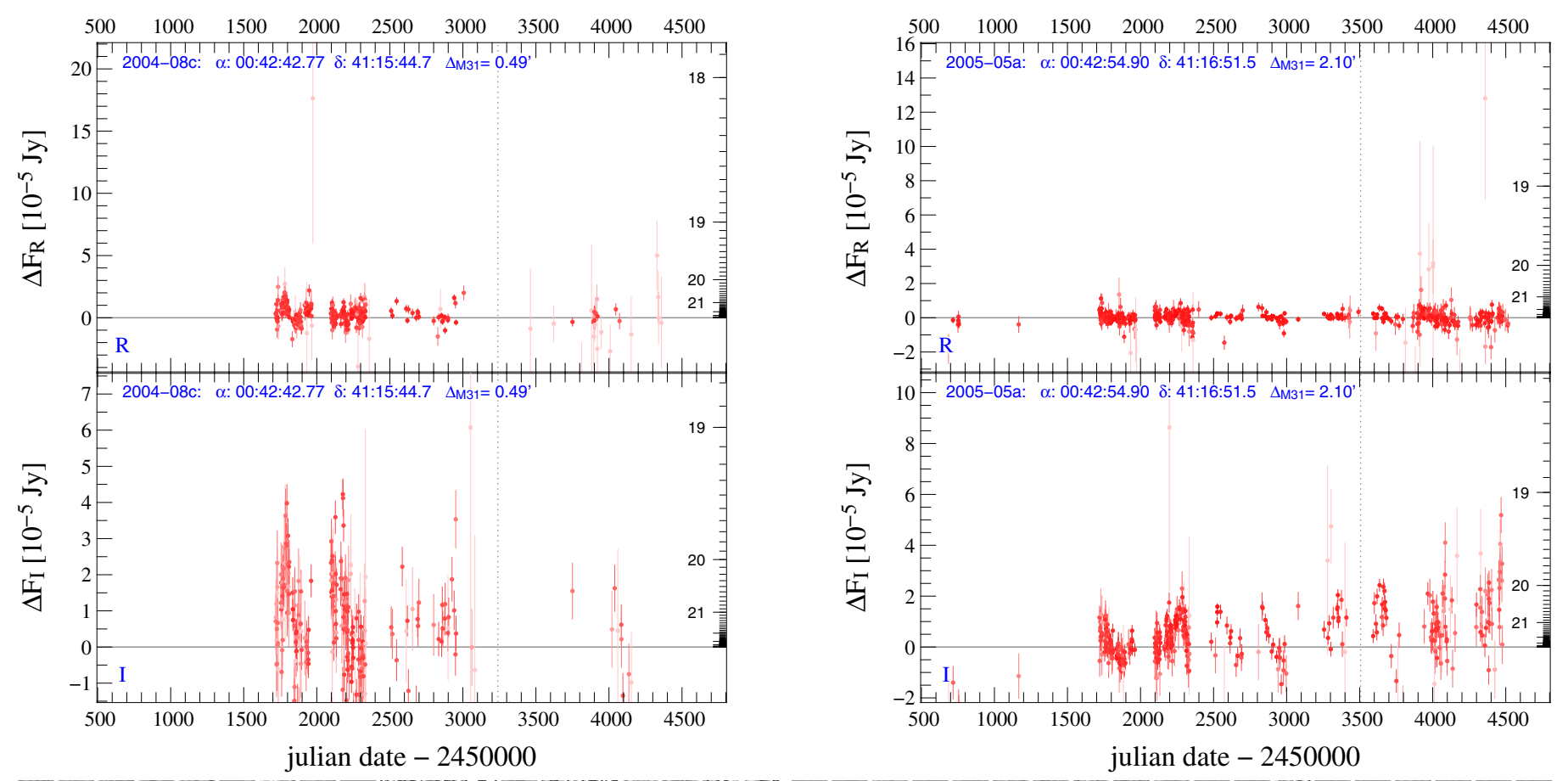

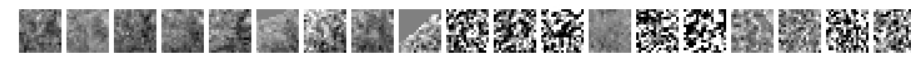

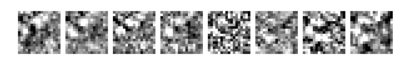

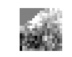

Fig. B.20. Light curve of 2004-08c and 2005-05a from WeCAPP data. 